\title{
Programação Dinâmica em Tempo Real para Processos de Decisão Markovianos com Probabilidades Imprecisas
}

Daniel Baptista Dias

\author{
DisSERTAÇÃO APRESENTADA \\ $\mathrm{AO}$ \\ Instituto DE MATEMÁticA E EstatísticA \\ DA \\ UniversidADE DE SÃO PAULO \\ PARA \\ OBTENÇÃO DO TÍTULO \\ $\mathrm{DE}$ \\ Mestre em CiênCIAS
}

Área de concentração: Ciência da Computação

Orientadora: Prof. Dra. Karina Valdivia Delgado 


\section{Programação Dinâmica em Tempo Real para Processos de Decisão Markovianos com Probabilidades Imprecisas}

Esta versão da dissertação contém as correções e alterações sugeridas pela Comissão Julgadora durante a defesa da versão original do trabalho, realizada em 28/11/2014. Uma cópia da versão original está disponível no

Instituto de Matemática e Estatística da Universidade de São Paulo.

Comissão Julgadora:

- Prof ${ }^{\mathrm{a}}$. Dr ${ }^{\mathrm{a}}$. Karina Valdivia Delgado (orientadora) - EACH-USP

- Prof ${ }^{\mathrm{a}}$. Dr ${ }^{\mathrm{a}}$. Leliane Nunes de Barros - IME-USP

- Prof. Dr. Fábio Gagliardi Cozman - POLI-USP 


\section{Agradecimentos}

Agradeço aos meus avós Manuel, Dirce, Anésio e Sebastiana que sempre lutaram, ao jeito deles, para que seus filhos e netos pudessem ter uma vida melhor.

Agradeço o pessoal que trabalhou diretamente comigo para a construção desta dissertação: a minha orientadora Karina, pela infinita paciência e por toda a ajuda que me deu ao longo da construção deste trabalho, principalmente em sua etapa final, a professora Leliane, por ter me ajudado com os primeiros passos no IME e por seus valiosos conselhos ao longo do mestrado e deste trabalho, ao Felipe Trevizan, que me ajudou muito a compreender melhor os modelos do Short-Sighted Stochastic Shortest Paths Problems (Short-Sighted SSP) e ao Scott Sanner que nos deu conselhos valiosos na montagem do artigo para o AI Journal e neste trabalho como um todo.

Agradeço aos meus amigos do laboratório do LIAMF, em especial ao Fábio, o Mijail, a Viviane, o Paulo, o Luiz, o Ricardo, o Felipe, o Ignasi, o Adalberto, a Érica e todo o pessoal que sempre me recebeu muito bem, que me apoiaram e ajudaram muito neste trabalho, mesmo não sabendo disso.

Agradeço ao pessoal do meu trabalho na MVar, em especial ao Alexandre, o Claudio, o Paulo, o Rodrigo, o TAM, a Renata, o Flamínio, a Viviane, a Nicole, o Gus e o Gabriel que me apoiaram e entenderam minhas ausências para terminar este trabalho. Ao pessoal da TSKF, em especial ao Luiz Fabiano, o Guilherme, o Fernando e a Paula, antes meus professores, agora meus colegas de treino e de aulas, que através do treino de Kung Fu me ajudaram a ter o equilíbrio necessário para terminar esta dissertação.

Também agradeço ao pessoal do curso de Sistemas de Informação da EACH-USP que se formou comigo, me incentivou a fazer o mestrado e sempre seguir em frente, em especial ao Cláudio, o Paolo, o Ernando, o Jorge, o Felipe, a Jaqueline, a Alexandra, a Camila e o Cristiano.

Por fim, o agradecimento mais importante vai para os meus pais Alex e Márcia, e meus irmãos Lucas e Mirian que acompanharam cada passo desta jornada e me apoiaram com um amor incondicional para que ela pudesse ser concluída. Sem vocês eu nunca chegaria até aqui. 


\section{Resumo}

DIAS, D. B. Programação dinâmica em tempo real para Processos de Decisão Markovianos com Probabilidades Imprecisas. 2014. 80 f. Dissertação (Mestrado) - Instituto de Matemática e Estatística, Universidade de São Paulo, São Paulo, 2014.

Em problemas de tomada de decisão sequencial modelados como Processos de Decisão Markovianos (MDP) pode não ser possível obter uma medida exata para as probabilidades de transição de estados. Visando resolver esta situação os Processos de Decisão Markovianos com Probabilidades Imprecisas (Markov Decision Processes with Imprecise Transition Probabilities, MDP-IPs) foram introduzidos. Porém, enquanto estes MDP-IPs se mostram como um arcabouço robusto para aplicações de planejamento no mundo real, suas soluções consomem muito tempo na prática. Em trabalhos anteriores, buscando melhorar estas soluções foram propostos algoritmos de programação dinâmica síncrona eficientes para resolver MDP-IPs com uma representação fatorada para as funções de transição probabilística e recompensa, chamados de MDP-IP fatorados. Entretanto quando o estado inicial de um problema do Caminho mais Curto Estocástico (Stochastic Shortest Path MDP, SSP MDP) é dado, estas soluções não utilizam esta informação. Neste trabalho será introduzido o problema do Caminho mais Curto Estocástico com Probabilidades Imprecisas (Stochastic Shortest Path MDP-IP, SSP MDP-IP) tanto em sua forma enumerativa, quanto na fatorada. Um algoritmo de programação dinâmica assíncrona para SSP MDP-IP enumerativos com probabilidades dadas por intervalos foi proposto por Buffet e Aberdeen (2005). Entretanto, em geral um problema é dado de forma fatorada, i.e., em termos de variáveis de estado e nesse caso, mesmo se for assumida a imprecisão dada por intervalos sobre as variáveis, ele não poderá ser mais aplicado, pois as probabilidades de transição conjuntas serão multilineares. Assim, será mostrado que a mudança do SSP MDP-IP enumerativo para o caso geral de um SSP MDP-IPs fatorado leva a uma mudança de resolução da função objetivo do Bellman backup de uma função linear para uma não-linear. Também serão propostos algoritmos enumerativos, chamados de RTDP-IP (Real-time Dynamic Programming with Imprecise Transition Probabilities), LRTDP-IP (Labeled Real-time Dynamic Programming with Imprecise Transition Probabilities), SSiPP-IP (Short-Sighted Probabilistic Planner with Imprecise Transition Probabilities) e LSSiPP-IP (Labeled Short-Sighted Probabilistic Planner with Imprecise Transition Probabilities) e fatorados chamados factRTDP-IP (factored RTDP-IP) e factLRTDP-IP (factored LRTDP-IP). Eles serão avaliados em relação aos algoritmos de programação dinâmica síncrona em termos de tempo de convergência da solução e de escalabilidade.

Palavras-chave: Planejamento Probabilístico, Processos de Decisão Markovianos com Probabilidades Imprecisas, Planejamento Robusto. 


\section{Abstract}

DIAS, D. B. Real-time dynamic programming for Markov Decision Processes with Imprecise Probabilities. 2014. 80 f. Dissertação (Mestrado) - Instituto de Matemática e Estatística, Universidade de São Paulo, São Paulo, 2014.

In sequential decision making problems modelled as Markov Decision Processes (MDP) we may not have the state transition probabilities. To solve this issue, the framework based in Markov Decision Processes with Imprecise Transition Probabilities (MDP-IPs) is introduced. Therefore, while MDP-IPs is a robust framework to use in real world planning problems, its solutions are time-consuming in practice. In previous works, efficient algorithms based in synchronous dynamic programming to solve MDP-IPs with factored representations of the probabilistic transition function and reward function, called factored MDP-IPs. However, given a initial state of a system, modeled as a Stochastic Shortest Path MDP (SSP MDP), solutions does not use this information. In this work we introduce the Stochastic Shortest Path MDP-IPs (SSP MDP-IPs) in enumerative form and in factored form. An efficient asynchronous dynamic programming solution for SSP MDP-IPs with enumerated states has been proposed by Buffet e Aberdeen (2005) before which is restricted to interval-based imprecision. Nevertheless, in general the problem is given in a factored form, i.e., in terms of state variables and in this case even if we assume interval-based imprecision over the variables, the previous solution is no longer applicable since we have multilinear parameterized joint transition probabilities. In this work we show that the innocuous change from the enumerated SSP MDP-IP cases to the general case of factored SSP MDP-IPs leads to a switch from a linear to nonlinear objectives in the Bellman backup. Also we propose assynchronous dynamic programming enumerativ algorithms, called RTDP-IP (Real-time Dynamic Programming with Imprecise Transition Probabilities), LRTDP-IP (Labeled Real-time Dynamic Programming with Imprecise Transition Probabilities), SSiPP-IP (Short-Sighted Probabilistic Planner with Imprecise Transition Probabilities) and LSSiPP-IP (Labeled Short-Sighted Probabilistic Planner with Imprecise Transition Probabilities), and factored algorithms called factRTDP-IP (factored RTDP-IP) and factLRTDP-IP (factored LRTDP-IP). There algorithms will be evaluated with the synchronous dynamic programming algorithms previously proposed in terms of convergence time and scalability.

Keywords: Probabilistic Planning, Markov Decision Processes with Imprecise Probabilities, Robust Planning. 


\section{Sumário}

$\begin{array}{ll}\text { Lista de Abreviaturas } & \text { ix }\end{array}$

Lista de Símbolos $\quad$ xi

Lista de Figuras $\quad$ xiii

1 Introdução $\quad 1$

1.1 Motivação . . . . . . . . . . . . . . . . . . . . . . . . . 3

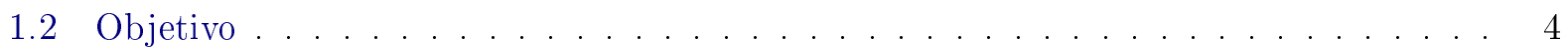

1.3 Principais Contribuições . . . . . . . . . . . . . . . . . . 4

1.4 Organização . . . . . . . . . . . . . . . . . . . . . . 5

2 Processos de Decisão Markovianos $\quad 7$

2.1 MDPs de Horizonte Finito e Infinito . . . . . . . . . . . . . . . . . 7

2.2 SSP MDP - MDPs de Horizonte Indeterminado . . . . . . . . . . . . . . . 8

2.3 SSP MDP Fatorados . . . . . . . . . . . . . . . . . . . . . . 10

2.4 Short-Sighted SSP MDPs $\ldots \ldots \ldots \ldots \ldots \ldots \ldots \ldots$

3 Soluções de SSP MDPs $r$

3.1 Soluções Síncronas para SSP MDPs . . . . . . . . . . . . . . . . . . . 13

3.1 .1 Iteração de Valor para SSP MDPs . . . . . . . . . . . . . . . . . 13

3.1 .2 Iteração de Política para SSP MDPs . . . . . . . . . . . . . . . . . 14

3.2 Programação Dinâmica Assíncrona para SSP MDPs . . . . . . . . . . . . . 15

3.2.1 RTDP - Programação Dinâmica em Tempo Real . . . . . . . . . . . . . . 15

3.2.2 LRTDP - Programação Dinâmica em Tempo Real Rotulada . . . . . . . . . . 18

3.2.3 SSiPP - Planejador Probabilístico Míope . . . . . . . . . . . . . . . . 19

3.2.4 LSSiPP - Planejador Probabilístico Míope Rotulado . . . . . . . . . . . . . . 22

3.3 Soluções para SSP MDP Fatorados . . . . . . . . . . . . . . . . . 22

3.3 .1 Diagramas de Decisão Algébricos. . . . . . . . . . . . . . . . 22

3.3.2 Algoritmos Síncronos e Assíncronos para SSP MDP Fatorados . . . . . . . . 23

4 Processos de Decisão Markovianos com Probabilidades Imprecisas 25

4.1 SSP MDP-IPs - MDP-IPs de Horizonte Indeterminado . . . . . . . . . . . . . . 25

4.2 Iteração de Valor: Solução Síncrona e Enumerativa para SSP MDP-IPs . . . . . . . 27

4.3 Outros arcabouços com modelos de transição imprecisas . . . . . . . . . . . . . 28

4.3 .1 BMDP - MDP Delimitado . . . . . . . . . . . . . . . 28 
4.3.2 MDP-ST - MDP com Transições Valoradas por Conjuntos . . . . . . . . . . 28

4.3.3 Relação entre MDP, BMDP, MDP-ST e MDP-IP . . . . . . . . . . . . . . 28

4.3.4 Planejamento Contingente e Abordagens Possibilísticas . . . . . . . . . . . . . 29

4.3 .5 Controle Robusto . . . . . . . . . . . . . . . . . . . . . 30

5 Processos de Decisão Markovianos com Probabilidades Imprecisas Fatorados 31

5.1 SSP MDP-IP Fatorado . . . . . . . . . . . . . . . . . . . . . . . 31

5.1.1 Independência Par Estado abstrato-Ação e Número de Conjuntos Credais . . 33

5.1.2 Gerando um SSP MDP-IP Enumerativo a partir de um SSP MDP-IP Fatorado 33

5.2 Diagramas de Decisão e SSP MDP-IPs Fatorados . . . . . . . . . . . . . . . . 34

5.2 .1 PADDs - ADDs Parametrizados . . . . . . . . . . . . . . . . 34

5.2 .2 O Algoritmo SPUDD-IP . . . . . . . . . . . . . . . . . 38

6 Programação Dinâmica Assíncrona para SSP MDP-IPs 41

6.1 O Algoritmo RTDP-IP . . . . . . . . . . . . . . . . . . . . . . . . . 41

6.1 .1 Amostragem de Estados no RTDP-IP . . . . . . . . . . . . . . . 42

6.1 .2 Convergência do RTDP-IP . . . . . . . . . . . . . . . . . . 44

6.2 O Algoritmo LRTDP-IP . . . . . . . . . . . . . . . . . . 46

6.3 O Algoritmo factRTDP-IP . . . . . . . . . . . . . . . . . . 46

6.4 O Algoritmo factLRTDP-IP . . . . . . . . . . . . . . . . . . 54

6.5 Os Algoritmos SSiPP-IP e LSSiPP-IP . . . . . . . . . . . . . . . . . . 54

6.5 .1 Short-Sighted SSP MDP-IPs _ . . . . . . . . . . . . . 54

$6.5 .2 \quad$ SSiPP-IP e LSSiPP-IP . . . . . . . . . . . . . . . . . . . 55

7 Experimentos $\quad 57$

7.1 Domínios . . . . . . . . . . . . . . . . . . . . 57

7.1 .1 Domínio Navigation . . . . . . . . . . . . . . . 57

7.1 .2 Domínio Triangle Tireworld . . . . . . . . . . . . . . . . . . 58

7.1 .3 Domínio SysAdmin . . . . . . . . . . . . . . . . . . . 58

7.2 Configuração dos Experimentos . . . . . . . . . . . . . . . . 58

7.3 Comparação entre SPUDD-IP, RTDP-IP, LRTDP-IP, factRTDP-IP e factLRTDP-IP 59

7.3.1 Taxa de Convergência do Estado Inicial . . . . . . . . . . . . . . . . . 59

7.3.2 Tempo de Convergência por Instância de Problema . . . . . . . . . . . . . . 61

7.3.3 Número de Chamadas ao Solver Multilinear . . . . . . . . . . . . . . . . . . 62

7.4 Comparação entre o LRTDP-IP e o LSSiPP-IP . . . . . . . . . . . . . . . . . 62

8 Conclusão e Trabalhos Futuros $\quad 69$

8.1 Resumo das Contribuições . . . . . . . . . . . . . . . . . . . . . . . . 70

8.2 Trabalhos Futuros . . . . . . . . . . . . . . . . . . 70

$\begin{array}{ll}\text { Referências Bibliográficas } & 73\end{array}$

Apêndice I - Convergência do RTDP-IP para MDP-IPs de Horizonte Infinito 77

Apêndice II - Descrição das ferramentas utilizadas nos experimentos $\quad 79$ 


\title{
Lista de Abreviaturas
}

\author{
ADD \\ Diagrama de Decisão Algébrico (Algebraic Decision Diagram) \\ BDD \\ Diagrama de Decisão Booleano (Boolean Decision Diagram) \\ DBN \\ Rede Bayesiana Dinâmica (Dynamic Bayesian Network) \\ $\mathrm{DCN}$ \\ Rede Credal Dinâmica (Dynamic Credal Network) \\ factLRTDP Programação Dinâmica em Tempo Real Rotulada \\ (factored Labeled Real-time Dynamic Programming) \\ factLRTDP-IP Programação Dinâmica em Tempo Real Rotulada com Probabilidades \\ Imprecisas Fatorada (factored Labeled Real-time Dynamic Programming with \\ Imprecise Transition Probabilities) \\ factRTDP Programação Dinâmica em Tempo Real Fatorada \\ (factored Real-time Dynamic Programming) \\ factRTDP-IP Programação Dinâmica em Tempo Real com Probabilidades Imprecisas \\ Fatorada (factored Real-time Dynamic Programming with Imprecise \\ Transition Probabilities) \\ IV Iteração de Valor \\ IP Iteração de Política \\ LRTDP Programação Dinâmica em Tempo Real Rotulada (Labeled Real-time \\ Dynamic Programming) \\ LRTDP-IP Programação Dinâmica em Tempo Real Rotulada com Probabilidades \\ Imprecisas (Labeled Real-time Dynamic Programming with Imprecise \\ Transition Probabilities) \\ LSSiPP Planejador Probabilístico Míope Rotulado (Labeled Short-Sighted \\ Probabilistic Planner) \\ LSSiPP-IP Planejador Probabilístico Míope Rotulado com Probabilidades Imprecisas \\ (Labeled Short-Sighted Probabilistic Planner with Imprecise Transition \\ Probabilities) \\ MDP \\ Processo de Decisão Markoviano (Markov Decision Process) \\ MDP-IP \\ Processo de Decisão Markoviano com Probabilidades Imprecisas \\ (Markov Decision Process with Imprecise Transition Probabilities) \\ PADD Diagrama de Decisão Algébrico Parametrizado (Parametrized Algebraic \\ Decision Diagram) \\ RTDP Programação Dinâmica em Tempo Real (Real-time Dynamic Programming) \\ RTDP-IP Programação Dinâmica em Tempo Real com Probabilidades Imprecisas \\ (Real-time Dynamic Programming with Imprecise Transition Probabilities)
}


SSiPP

SSiPP-IP

Short-Sighted SSP MDP

Short-Sighted SSP MDP-IP

SSP MDP

SSP MDP-IP

SPUDD

SPUDD-IP
Planejador Probabilístico Míope (Short-Sighted Probabilistic Planner)

Planejador Probabilístico Míope com Probabilidades Imprecisas

(Short-Sighted Probabilistic Planner with Imprecise Transition

Probabilities)

Problema do Caminho mais Curto Estocástico Míope (Short-Sighted Shortest Path MDP)

Problema do Caminho mais Curto Estocástico Míope com

Probabilidades Imprecisas (Short-Sighted Shortest Path MDP with

Imprecise Transition Probabilities)

Problema do Caminho mais Curto Estocástico (Stochastic Shortest

Path $M D P$ )

Problema do Caminho mais Curto Estocástico com Probabilidades

Imprecisas (Stochastic Shortest Path with Imprecise Transition Probabilities)

Planejamento Estocástico utilizando Diagramas de Decisão (Stochastic Planning using Decision Diagrams)

Planejamento Estocástico utilizando Diagramas de Decisão com Probabilidades Imprecisas (Stochastic Planning using Decision Diagrams with Imprecise Transition Probabilities) 


\section{Lista de Símbolos}

$\epsilon \quad$ Erro máximo permitido para a convergência de um algoritmo

$\gamma \quad$ Fator de desconto

$\pi \quad$ Política

$\pi^{*} \quad$ Política ótima

$\varphi \quad$ Conjunto de restrições sobre os parâmetros de probabilidades

$A \quad$ Conjunto discreto e finito de ações de um problema

C Função custo

$C_{D D} \quad$ Função custo representada como uma ADD

$C_{s, t} \quad$ Função custo de um Short-Sighted SSP MDP centrado em $s$ com profundidade $t$

$G \quad$ Conjunto de estados meta de um problema

$G_{s, t} \quad$ Conjunto de estados meta de um Short-Sighted SSP MDP centrado em $s$ com profundidade $t$

$H_{m d p} \quad$ Histórico de um MDP

$H \quad$ Heurística utilizada nos Short-Sighted SSP MDP

$\mathcal{K} \quad$ Conjunto credal de transição

$P \quad$ Função de probabilidade de transição entre estados

$P_{D D} \quad$ Função de probabilidade de transição entre estados representada como uma ADD

$p a_{a}\left(X_{i}\right) \quad$ Pais da variável $X_{i}$ dado a ação $a$

$R \quad$ Função recompensa

$S \quad$ Conjunto de estados de um problema

$S_{s, t} \quad$ Conjunto de estados de um Short-Sighted SSP MDP centrado em $s$ com profundidade $t$

$V \quad$ Função valor

$V^{h} \quad$ Função valor no horizonte $h$

$V^{t} \quad$ Função valor no estágio $t$

$V^{\pi} \quad$ Função valor para a política $\pi$

$V^{*} \quad$ Função valor ótima

$V_{D D} \quad$ Função valor representada como uma ADD

$X_{i} \quad$ Valor da variável $X_{i}$

$Y \quad$ Conjunto de variáveis de teste

$Z \quad$ Conjunto de parâmetros

$\vec{p} \quad$ Vetor de parâmetros de probabilidades

$\vec{w} \quad$ Atribuição parcial de variáveis

$\vec{X} \quad$ Vetor de variáveis binárias de um MDP-IP

$\vec{x} \quad$ Estado fatorado

$\vec{y} \quad$ Atribuição de variáveis 


\section{Lista de Figuras}

3.1 Operações realizadas no algoritmo RTDP . . . . . . . . . . . . . . 16

3.2 Exemplo de uma execução do planejador Non-Learning-Planner $\ldots \ldots$. . . . . . 20

3.3 Exemplo de função custo representada como um ADD . . . . . . . . . . . . . . . 23

4.1 Exemplo de uma função de transição probabilística parametrizada no SSP MDP-IP. $\quad 26$

4.2 Relacionamento entre o MDP-IP e suas subclasses (Trevizan et al., 2007). . . . . . . 29

5.1 Rede Credal Dinâmica para a ação $a_{1} \in A \ldots \ldots \ldots \ldots$. . . . . . . . 32

5.2 Matriz de transição parametrizada completa $P\left(\cdot \mid s_{i}, a_{1}\right) \ldots \ldots \ldots \ldots \ldots$

5.3 Representação usando PADDs das tabelas de probabilidade condicional. . . . . . . . 35

5.4 Um exemplo da operação de restrição sobre o PADD . . . . . . . . . . . . . . . . . 35

5.5 Avaliação do PADD para a função $P_{D D}\left(X_{2}^{\prime} \mid X_{1}, X_{2}, a_{1}\right) \ldots \ldots \ldots \ldots$

5.6 Avaliação parcial do PADD $P_{D D}\left(X_{2}^{\prime} \mid X_{1}, X_{2}, a_{1}\right) \operatorname{com} \vec{w}=\left\{X_{1}=1, X_{2}=1\right\} \ldots . .37$

5.7 Substituição de parâmetros do PADD para a função $P_{D D}\left(X_{1}^{\prime} \mid X_{1}, a_{1}\right) \ldots \ldots$

5.8 Exemplo da função MaxParameterOut. . . . . . . . . . . . . . . . . . . . . . 38

5.9 Eliminação de variáveis no SPUDD-IP . . . . . . . . . . . . . . . . . 39

6.1 Bellman update do RTDP-IP e a amostragem do próximo estado. . . . . . . . . . . 44

6.2 Um exemplo de conjunto credal de transição representado pela área em cinza. . . . . 45

6.3 PADD resultante da operação $p E v a l P A D D\left(P_{D D}\left(X_{i}^{\prime} \mid p a_{a}\left(X_{i}^{\prime}\right), a\right), \vec{x}\right) \ldots \ldots \ldots$

6.4 Eliminação de variáveis no factRTDP-IP. . . . . . . . . . . . . . . . . . . 48

6.5 Exemplo das operações com PADDs para calcular a Equação (6.7). . . . . . . . . . 49

6.6 Inserção de $V^{t+1}(\vec{x})$ em $V_{D D}^{t}(\vec{X}) \ldots \ldots \ldots \ldots \ldots \ldots \ldots \ldots$

6.7 Amostragem das variáveis de estado $X_{1}^{\prime}$ e $X_{2}^{\prime} \ldots \ldots \ldots \ldots \ldots \ldots$

7.1 Valor do estado inicial no SPUDD-IP, (L)RTDP-IP e fact(L)RTDP-IP. . . . . . . . 60

7.2 Tempo de convergência do SPUDD-IP, LRTDP-IP e o factLRTDP-IP. . . . . . . . 63

7.3 Tempo e chamadas ao solver para o SPUDD-IP, o LRTDP-IP e o factLRTDP-IP. . . 64

7.4 Valor do estado inicial do LSSiPP-IP e LRTDP-IP. . . . . . . . . . . . . . . . . 65

7.5 Tempo de convergência do LSSiPP-IP e LRTDP-IP. . . . . . . . . . . . . . . 66

7.6 Tempo de convergência e chamadas ao solver para o LSSiPP-IP e LRTDP-IP. . . . . 67

7.7 Tempo percentual de chamadas ao solver para o LSSiPP-IP $(\mathrm{t}=5)$ e LRTDP-IP. . . . 68 


\section{Capítulo 1}

\section{Introdução}

Um Processo de Decisão Markoviano (Puterman, 1994) (MDP) codifica a interação entre um agente e seu ambiente: a cada estágio o agente decide executar uma ação (com efeitos probabilísticos) que o levam a um próximo estado e geram uma recompensa. A meta do agente é maximizar a recompensa esperada sobre uma sequência de ações. Os MDPs foram utilizados como um modelo padrão para problemas de planejamento probabilístico onde a incerteza é representada por uma matriz com as probabilidades de transição entre os estados para cada ação possível.

Existem três tipos importantes de MDPs: o de horizonte finito, onde o agente deve agir um número $H \neq \infty$ de passos; o de horizonte infinito, onde o agente deve agir por $H=\infty$ passos; e o de horizonte indefinido, onde o agente deve agir por um número finito porém desconhecido de passos. Um exemplo deste último caso é o MDP do Caminho mais Curto Estocástico (Stochastic Shortest Path MDP, SSP MDP). Algumas soluções eficientes para os MDPs exploram tanto a estrutura fatorada na representação do MDP (como pode ser visto em Boutilier et al. (1999), Guestrin et al. (2003), Hoey et al. (1999), St-Aubin et al. (2000)), quanto o conhecimento do estado inicial de um SSP MDP com a finalidade de calcular a solução considerando os estados alcançáveis via programação dinâmica assíncrona (Barto et al., 1995, Bonet e Geffner, 2003). As soluções baseadas em programação dinâmica podem ser síncronas ou assíncronas. Uma solução de programação dinâmica síncrona é aquela onde todos os estados tem o seu valor atualizado durante cada iteração do algoritmo. Já uma solução de programação dinâmica assíncrona é aquela onde apenas uma parcela dos estados tem o seu valor atualizado durante uma iteração do algoritmo.

Entretanto, em vários problemas reais é impossível se obter uma representação precisa das probabilidades de transição de um MDP. Isto pode ocorrer por diversas razões como (a) informações imprecisas ou conflitantes dadas por especialistas (Givan et al., 2000), (b) o fato de muitas vezes haver dados insuficientes para se estimar modelos de transições precisos (Delgado et al., 2011), (c) o problema de ter probabilidades de transição não estacionárias devido a falta de informações sobre os estados ou (d) a ocorrência de eventos imprevisíveis (Witwicki et al., 2013). Nestas situações o agente deve tomar decisões contra as possíveis escolhas da Natureza.

Exemplo 1. Em um sistema de navegação de robôs a probabilidade de alcançar uma determinada localização depois do robô se mover do ambiente pode mudar ao longo do tempo devido as condições do ambiente (como o clima da região onde o robô se localiza e as condições da via em que está trafegando), que podem fazer a navegação por alguns caminhos se tornarem mais difíceis e sujeitas a falhas. Em geral é difícil modelar com acurácia todas estas mudanças, pois muitas delas dependem 
de fatores externos. Neste caso pode ser melhor ter uma política otimizada sob um conjunto de probabilidades possíveis com o intuito de ter um comportamento robusto.

Exemplo 2. No campo da genética é possível modelar uma rede reguladora de genes e um conjunto de ações (intervenções terapêuticas) como um MDP (Bryce et al., 2010, Datta et al., 2003, Pal et al., 2008). Estes tratamentos previnem que a rede chegue a estados indesejáveis, associados com doenças. Porém, modelar o MDP com as probabilidades de transição exatas pode não ser possível quando apenas algumas amostras de dados estão disponíveis ou ainda devido a existência de eventos exógenos. Neste caso, um modelo com transições imprecisas pode ser usado para calcular uma política robusta para a intervenção terapêutica.

Os MDPs com probabilidades imprecisas (MDP-IP) foram introduzidos por Satia e Lave Jr. (1970) e White III e El-Deib (1994) para se criar modelos ótimos de tomada de decisão sequencial na presença de incertezas nas transições de estados. Um MDP-IP é um processo de decisão sequencial dotado de um conjunto de estados, ações e recompensas como um MDP, porém com uma diferença: nele as probabilidades de transição imprecisas são especificadas, através de matrizes de transição de estados parametrizadas. Por exemplo, a probabilidade de ir do estado $s_{1}$ para o estado $s_{2}$, após executar a ação $a_{1}$ pode ser dada através do parâmetro $p_{1}$ sujeito a uma restrição como $0 \leq p_{1} \leq 0.75$. Desta maneira ao invés de haver apenas uma medida de probabilidade para esta transição há um conjunto de medidas de probabilidades para um determinado par estado-ação sujeito a um conjunto de restrições $\varphi$ sobre $k$ parâmetros definidos por $\vec{p} \in[0,1]^{k}$.

Enquanto um MDP-IP se mostra como um arcabouço robusto para aplicações reais de planejamento, suas soluções demandam muito tempo na prática (Delgado et al., 2011) pois implicam em um problema de otimização mais complexo que o de um MDP: nele a meta do agente é maximizar uma recompensa esperada sobre uma sequência de ações, considerando o pior caso das probabilidades de transição imprecisas.

As soluções estado-da-arte para MDP-IPs estão baseadas em representações fatoradas e em programação dinâmica síncrona (Delgado et al., 2011, Satia e Lave Jr., 1970, White III e El-Deib, 1994). A eficiência da solução fatorado de um MDP-IP, o SPUDD-IP (Delgado et al., 2011), se deve ao uso de PADDs (Delgado et al., 2011), um diagrama de decisão algébrico com expressões parametrizadas em suas folhas, para calcular com eficiência uma solução exata conseguindo melhorar até em duas ordens de magnitude a velocidade de convergência em relação as técnicas de iteração de valor exatas para MDP-IPs.

Um objetivo deste trabalho é melhorar ainda mais o desempenho das soluções para MDP-IPs. Em particular, explorando soluções assíncronas para uma classe mais geral de MDP-IPs, chamada de MDP-IP do Caminho mais Curto Estocástico (Stochastic Shortest Path MDP-IP) ou SSP MDP-IP.

Uma solução de programação dinâmica assíncrona para SSP MDPs (Bertsekas, 1982) de interesse particular é a programação dinâmica em tempo real (RTDP), proposto por Barto et al. (1995), como pode ser evidenciado por diversos trabalhos recentes (Bonet e Geffner, 2003, McMahan et al., 2005, Sanner et al., 2009, Smith e Simmons, 2006). Esta abordagem atualiza as informações dos estados através de uma série de rodadas, chamadas de trials, resultantes da simulação de uma política gulosa. Este algoritmo tem as seguintes vantagens para a busca de soluções para SSP MDPs:

(a) Bom desempenho em qualquer instante: este algoritmo pode ser interrompido em qualquer instante, podendo produzir políticas melhores a medida que é executado por mais tempo; 
(b) Otimalidade sem exploração exaustiva: focando as buscas baseadas em trials que visitam os estados alcançáveis a partir do estado inicial e utilizando heurísticas informativas, esta classe de algoritmos pode obter uma política ótima visitando apenas uma fração do espaço de estados.

Uma versão melhorada do RTDP, chamada de LRTDP, rotula estados resolvidos, i.e., estados para o qual a função valor já convergiu (considerando um erro residual). Um estado é rotulado como resolvido quando seu grafo guloso (conjunto de estados alcançados através dele com uma política gulosa) também estão marcados como resolvidos. Com estas mudanças, três vantagens adicionais podem ser consideradas:

(c) O algoritmo agora tem uma condição de parada, que ocorre quando o estado inicial foi marcado como resolvido, garantindo que todos os estados relevantes foram resolvidos;

(d) Atualiza os estados do grafo guloso para cada estado visitado em um trial, garantindo que os estados relevantes, mesmo aqueles com pouca probabilidade, sejam atualizados; e

(e) Os trials podem ser finalizados mais cedo caso alcancem estados resolvidos, evitando visitas desnecessárias a estados que já convergiram.

Baseados nestas idéias, vários trabalhos buscam melhorar estes métodos de programação dinâmica assíncrona de forma a eles tornarem mais eficientes para a resolução de problemas maiores e com o caráter mais prático. Um exemplo pode ser visto no algoritmo Glutton (Kolobov et al., 2012), que ficou em segundo lugar no IPPC-2011 ${ }^{1}$, pelos algoritmos factRTDP e factLRTDP, propostos por Holguin (2013) que obtiveram resultados comparáveis aos dos competidores do IPPC-2011 e pelos algoritmos SSiPP e LSSiPP (Trevizan, 2013) que também tiveram resultados comparáveis aos dos competidores dos IPPCs recentes.

Um algoritmo eficiente de programação dinâmica assíncrona para SSP MDP-IPs com estados enumerativos já foi proposto anteriormente, que representa as imprecisões de probabilidade como intervalos (Buffet e Aberdeen, 2005). Entretanto, em geral um problema é dado de forma fatorada, i.e., em termos de variáveis de estado. Neste caso, mesmo se for assumido imprecisão dada por intervalos sobre as variáveis, o algoritmo anterior não será aplicável visto que existem probabilidades de transição conjuntas parametrizadas multilineares. Neste trabalho é apresentada a primeira solução assíncrona para SSP MDP-IPs dadas em termos de um conjunto de restrições gerais que também pode ser aplicada a SSP MDP-IPs fatorados.

\subsection{Motivação}

Motivados pelo sucesso da programação dinâmica assíncrona para SSP MDPs, em especial dos algoritmos (L)RTDP e (L)SSiPP, e verificando na literatura que não houve iniciativas em se desenvolver este tipo de algoritmo para SSP MDP-IP descritos em termos de um conjunto geral de restrições, este trabalho pretende criar novos algoritmos de programação dinâmica assíncrona para SSP MDP-IPs.

\footnotetext{
${ }^{1}$ International Probabilistic Planning Competition (http://users.cecs.anu.edu.au/ ssanner/IPPC_2011), realizada a cada 2 anos.
} 
Para isto deve-se pensar em como adaptar as características dos algoritmos para o modelo de SSP MDP-IP. O desafio de estender os algoritmos RTDP e o LRTDP para resolver problemas de SSP MDP-IP são: (i) como garantir a convergência de soluções de programação dinâmica assíncrona para SSP MDP-IPs? (ii) como amostrar o próximo estado no trial dadas as probabilidades imprecisas? Já no caso de estender os algoritmos SSiPP e LSSiPP, as duas primeiras perguntas também se aplicam a eles e uma terceira é adicionada: (iii) como criar os Short-Sighted SSP MDP-IPs a partir de SSP MDP-IPs?

\subsection{Objetivo}

O objetivo deste trabalho de mestrado é:

- Propor novos algoritmos assíncronos para resolver SSP MDP-IPs tanto enumerativos quanto fatorados, estendendo os algoritmos (L)RTDP e (L)SSiPP para lidar com um conjunto de probabilidades no lugar de probabilidades precisas.

\subsection{Principais Contribuições}

As principais contribuições deste trabalho são:

- Programação dinâmica assíncrona para SSP MDP-IPs: Foi proposta a primeira solução assíncrona para SSP MDP-IPs através da extensão do algoritmo existente para SSP MDPs, chamado RTDP-IP.

- Métodos de amostragem para o próximo estado: Foram propostos três possíveis métodos para escolher as medidas de probabilidade para amostrar o próximo estado no RTDP-IP, que são: (1) medidas válidas para o melhor no pior caso das probabilidades; (2) medidas de probabilidade aleatórias; e (3) medidas de probabilidade válidas pré definidas. É mostrado, que apesar da imprecisão sobre as probabilidades de transição, o RTDP-IP (com qualquer um dos três métodos) converge para uma solução robusta.

- Programação dinâmica assíncrona fatorada para SSP MDP-IPs: Foram propostas duas versões fatoradas do algoritmo (L)RTDP-IP, chamados factRTDP-IP e factLRTDP$I P$, que usam diagramas de decisão algébricos parametrizados (PADDs) para representar e atualizar eficientemente a função valor.

- Programação dinâmica assíncrona fatorada para Short-Sighted SSP MDP-IP: É mostrado como criar um Short-Sighted SSP MDP-IP a partir de SSP MDP-IPs e também são apresentados dois algoritmos que os resolvem: o SSiPP-IP e o LSSiPP-IP.

- Melhoria em termos de convergência em relação ao estado-da-arte: É mostrado empiricamente que os algoritmos LRTDP-IP e factLRTDP-IP tem uma velocidade de convergência em três ordens de magnitude maior que as soluções exatas estado-da-arte para domínios com matrizes de transição que não são densas. Portanto, na prática, é mostrado que os métodos assíncronos são mais eficientes que a Iteração de Valor síncrona e que permitem resolver instâncias maiores de problemas SSP MDP-IPs, algo que os algoritmos síncronos não 
eram capazes de resolver. Apesar do ganho de desempenho obtido pela Iteração de Valor fatorada (Delgado et al., 2011) em relação a Iteração de Valor enumerativa para MDP-IPs, o fact $(L) R T D P-I P$ não consegue ter um ganho de desempenho em relação a sua versão enumerativa, i.e., (L)RTDP-IP.

- Comparação entre diferentes tipos de escolha de probabilidades: Uma comparação empírica é feita entre os três métodos de se escolher as medidas de probabilidade para amostrar o próximo estado. Os resultados mostram que não há diferença significante em termos de tempo entre os três métodos.

\subsection{Organização}

Este trabalho está organizado da seguinte forma: no Capítulo 2 são mostradas as definições de SSP MDPs e Short-Sighted SSP MDPs. No Capítulo 3 alguns métodos de resolução de SSP MDPs utilizando programação dinâmica síncrona e assíncrona são apresentados. Já no Capítulo 4 são definidos os SSP MDP-IPs enumerativos e é mostrado como criar Short-Sighted SSP MDP-IPs enquanto no Capítulo 5 são mostrados os SSP MDP-IPs fatorados. No Capítulo 6 são propostos um conjunto de algoritmos assíncronos para a resolução de SSP MDP-IPs. No Capítulo 7 são mostrados as comparações feitas entre os algoritmos propostos neste trabalho e o algoritmo exato estado-da-arte. Por fim, no Capítulo 8 são mostradas as conclusões deste trabalho. 


\section{Capítulo 2}

\section{Processos de Decisão Markovianos}

$\mathrm{Na}$ área de Planejamento Probabilístico, uma forma de lidar com incertezas no resultado da execução de ações em um determinado ambiente é modelar o problema de planejamento utilizando um Processo de Decisão Markoviano (MDP, do inglês Markov Decision Process).

Através deste arcabouço é possível modelar um problema de tomada de decisão sequencial sob incerteza em um ambiente completamente observável (i.e., o agente tem conhecimento do estado atual do ambiente). A interação com o ambiente acontece de maneira sequencial, decorrendo em estágios (ou etapas), onde a cada estágio o agente escolhe e executa uma determinada ação alterando o estado do ambiente e recebendo uma determinada recompensa ou incorrendo em um determinado custo (recompensa negativa). Neste modelo o objetivo do agente é tomar as decisões que busquem minimizar o custo esperado, tornando este problema em um problema de otimização.

Neste capítulo são definidos formalmente os MDPs de horizonte finito, infinito e indeterminado (SSP MDP) e também são definidos os SSP MDPs fatorados e os Short-Sighted SSP MDPs (Trevizan, 2013).

\subsection{MDPs de Horizonte Finito e Infinito}

Um MDP é definido como uma tupla $\mathcal{M}=\langle S, A, R, P\rangle$, em que:

- $S$ é um conjunto finito de estados do sistema;

- $A$ é um conjunto finito de ações que o agente pode executar;

- $R: S \times A \rightarrow \mathbb{R}$ é a função de recompensa que o agente recebe ao executar uma ação $a \in A$ no estado $s \in S$;

- $P\left(s^{\prime} \mid s, a\right)$ define a probabilidade de transição de se alcançar um estado $s^{\prime} \in S$ a partir de um estado $s \in S$, executando a ação $a \in A$.

Neste modelo a tomada de decisões e execução de ações definem um histórico $H_{m d p}$, que é uma sequência de tuplas composta por uma ação e um estado, que define o comportamento do agente em cada instante de tempo (estágio). O histórico $H_{m d p}$ até o estágio $t$ é dado por:

$$
H_{m d p}=\left\langle\left\langle s^{0}, a^{0}\right\rangle,\left\langle s^{1}, a^{1}\right\rangle,\left\langle s^{2}, a^{2}\right\rangle, \cdots,\left\langle s^{t}, a^{t}\right\rangle\right\rangle
$$


Para auxiliar o agente a quantificar a qualidade da sua tomada de decisão, pode-se definir uma função valor $V_{h}: H_{m d p} \rightarrow \mathbb{R}$, que mapeia um histórico do sistema em um número real.

Esta quantificação depende do horizonte do sistema, i.e., a quantidade de estágios de decisão que o mesmo terá. Um problema pode ter um horizonte finito, onde o agente tem um número finito de $T$ estágios para executar suas ações ou um horizonte infinito onde o agente tem infinitos estágios $(T=\infty)$ para executar suas ações.

Em problemas com horizonte finito a função valor para um histórico $H_{m d p}$ pode ser definida simplesmente como a somatória das recompensas obtidas em cada estágio (recompensa total do histórico):

$$
V_{h}\left(H_{m d p}\right)=\sum_{t=0}^{T} R\left(s^{t}, a^{t}\right) .
$$

Já no caso do horizonte infinito, o histórico do agente precisa ser avaliado sob uma trajetória infinita. Para isto, o valor precisa ser limitado de alguma forma, caso contrário, históricos com boas tomadas de decisões e históricos com más tomadas de decisões estariam em condições iguais de comparação. Para limitar a função valor o modelo considera o fator de desconto $\gamma$ limitado ao intervalo $[0,1]$. Desta forma, o valor pode ser calculado por:

$$
V_{h}\left(H_{m d p}\right)=\sum_{t=0}^{\infty} \gamma^{t} R\left(s^{t}, a^{t}\right) .
$$

A solução de um MDP é uma política $\pi: S \rightarrow A$ que define para cada estado uma ação que otimiza um dado critério de desempenho. O critério mais usado é maximizar a recompensa esperada total. O valor de uma política $\pi$ no estado $s, V_{\pi}(s)$, é definido como a recompensa esperada total que a política incorre começando no estado $s_{0}$ :

$$
V_{\pi}(s)=E_{\pi}\left[\sum_{t=0}^{T} \gamma^{t} r_{t} \mid s_{0}=s\right],
$$

em que $r_{t}$ é a recompensa no instante $t$ quando o agente está no estado $s_{t}$ e executa a ação $\pi\left(s_{t}\right)$. Para MDPs de horizonte finito consideramos $\gamma=1$.

Uma solução para um MDP é a política ótima $\pi^{*}$ que fornece o valor maximal para cada estado, i.e., $V_{\pi^{*}}(s) \geq V_{\pi^{\prime}}(s), \forall s, \pi^{\prime}$.

\subsection{SSP MDP - MDPs de Horizonte Indeterminado}

Além dos MDPs apresentados, existem problemas com horizonte finito porém desconhecido que são chamados de problemas de horizonte indeterminado. Um tipo de MDP de horizonte indeterminado é o Stochastic Shortest Path MDP (SSP MDP). Nestes problemas cada política ótima leva o sistema a algum estado meta (Bertsekas e Tsitsiklis, 1991). Formalmente é preciso definir algumas restrições em um MDP de horizonte indeterminado, de forma a ter uma garantia a priori da existência de uma política ótima. MDPs de horizonte finito e infinitos são casos especiais de SSP MDPs (Bertsekas e Tsitsiklis, 1996).

O SSP MDP é um MDP com um estado inicial $s_{0}$, um conjunto de estados meta $G \in S$, em que todas as ações executadas nos estados meta levam a ele mesmo com recompensa 0 e para os 
outros estados deste modelo as recompensas imediatas são negativas para toda ação. Assim para SSP MDPs é mais apropriado trabalhar com custo no lugar de recompensa.

Formalmente, um SSP MDP (Bertsekas e Tsitsiklis, 1991), é uma tupla $\left\langle S, A, C, P, G, s_{0}\right\rangle$ em que:

- $S$ é um conjunto finito de estados;

- $A$ é um conjunto finito de ações;

- $C: S \times A \rightarrow \mathbb{R}^{+}$é uma função de custo;

- $P\left(s^{\prime} \mid s, a\right)$ define a probabilidade de transição de se alcançar um estado $s^{\prime}$ a partir de um estado $s \in S$, executando a ação $a \in A$;

- $G \subseteq S$ é um conjunto de estados meta, definidos como estados de absorção ${ }^{1}$. Para cada $s \in G$, $P(s \mid s, a)=1$ e $C(s, a)=0$ para todo $a \in A ; \mathrm{e}$

- $s_{0} \in S$ é o estado inicial.

Os SSP MDPs também devem satisfazer os seguintes pressupostos (Bertsekas e Tsitsiklis, 1991):

Pressuposto 2.2.1. Cada $s \in S$ deve ter ao menos uma política apropriada, i.e., uma política que garante que um estado meta é alcançado com probabilidade 1.

Pressuposto 2.2.2. Cada politica imprópria deve ter custo $\infty$ em todos os estados que não podem alcançar a meta com probabilidade 1

O Pressuposto 2.2.2 é verdadeiro nesta definição de SSP MDP, uma vez que os custos são estritamente positivos.

A solução para um SSP MDP é uma política ótima e parcial enraizada em $s_{0}$, i.e., uma política que permite que o agente alcance a meta pelo caminho menos custoso a partir de $s_{0}$ e é definida para cada estado que pode ser alcançado a partir de $s_{0}$.

A função valor é definida como o mapeamento $V: S \rightarrow \mathbb{R} \cup \infty$. O valor de uma política $\pi$ no estado $s, V_{\pi}(s)$, é definido como o custo esperado total iniciando em $s$ e seguindo $\pi$, i.e.:

$$
V_{\pi}(s)=E_{\pi}\left[\sum_{t=0}^{\infty} c_{t} \mid s_{0}=s\right],
$$

em que $c_{t}$ é o custo incorrido no tempo $t$ quando o agente está no estado $s_{t}$ e executa a ação $\pi\left(s_{t}\right)$.

Para se encontrar a solução ótima de um SSP MDP é necessário encontrar uma política $\pi^{*}$ que minimiza $V_{\pi}$. A função valor ótima $V^{*}=V_{\pi^{*}}$ é definida como $V^{*}=\min _{\pi} V_{\pi}$, que também satisfaz a seguinte condição para todo $s \in S$ :

$$
V^{*}(s)=\min _{a \in A}\left\{C(s, a)+\sum_{s^{\prime} \in S} P\left(s^{\prime} \mid s, a\right) V^{*}\left(s^{\prime}\right)\right\},
$$

Esta equação é também conhecida como a equação de Bellman para SSP MDPs. O interesse deste trabalho é propor algoritmos para uma extensão destes SSP MDPs.

\footnotetext{
${ }^{1}$ Estados em que para qualquer ação executada o sistema permanecerá no mesmo estado
} 


\subsection{SSP MDP Fatorados}

Uma outra maneira de representar um SSP MDP é, ao invés de representar um estado com informações autocontidas, representar os estados em termos de variáveis de estados. Esta representação é interessante, pois muitos problemas de planejamento são descritos por estas variáveis.

Assim, um SSP MDP fatorado é um SSP MDP onde os estados $\vec{x}$ são especificados como uma atribuição conjunta para um vetor $\vec{X}$ de $n$ variáveis de estado $\left(X_{1}, \ldots, X_{n}\right)$ e as funções de transição são representadas utilizando as Redes Bayesianas Dinâmicas (DBN, do inglês Dynamic Bayesian Networks) (Dean e Kanazawa, 1990). A função custo e a função valor também são dadas em termos de um subconjunto de $\left\{X_{1}, \cdots, X_{n}\right\} . x_{i}$ é usado para representar o valor atribuído para uma variável de estado $X_{i}$.

Para definir uma Rede Bayesiana Dinâmica, primeiro é necessário ter o conceito de dependência entre variáveis.

\section{Definição 2.3.1. (Pais de uma variável)}

Dada a ação a, o valor da variável $X_{i}$ depende do conjunto de variáveis $\left\{X_{j}, X_{k}, X_{l}, \ldots\right\}$ (possivelmente vazio). Este conjunto de variáveis é chamado de "pais" de $X_{i}$ a respeito da ação a denotada por $p a_{a}\left(X_{i}\right)$.

\section{Definição 2.3.2. (Rede Bayesiana Dinâmica - DBN (Dean e Kanazawa, 1990))}

Em uma Rede Bayesiana Dinâmica (DBN) (Dean e Kanazawa, 1990), dada uma açẫo a $\in A$, tem-se um grafo dirigido acíclico de duas camadas onde os nós da primeira camada representam as variáveis $X_{i}$ do estado atual, relacionados com os nós da segunda camada, que representam as variáveis do próximo estado $X_{i}^{\prime}$. Neste grafo arestas são permitidas entre os nós da primeira camada com a segunda camada e também entre nós da segunda camada. Os pais de $X_{i}, p a_{a}\left(X_{i}\right)$, podem ser identificados no grafo através dos nós que possuem uma aresta incidente em $X_{i}^{\prime}$. Uma suposição é feita neste grafo considerando a seguinte condição de Markov: uma variável $X_{i}$ é condicionalmente independente de seus não-descendentes não-pais dados seus pais, implicando na seguinte fatoração das probabilidades de transição:

$$
P\left(\vec{x}^{\prime} \mid \vec{x}, a\right)=\prod_{i=1}^{n} P\left(x_{i}^{\prime} \mid p a_{a}\left(X_{i}^{\prime}\right), a\right) .
$$

\subsection{Short-Sighted SSP MDPs}

Um SSP MDP com muitos estados muitas vezes não pode ser resolvido diretamente por um planejador. Em (Trevizan, 2013) foram propostas soluções que segmentam o SSP MDP em diversos subproblemas, mais fáceis de serem resolvidos chamadas Short-Sighted SSP MDPs.

A idéia de Trevizan (2013) para resolver um problema maior (SSP MDP) dado seu estado inicial, é criar um subproblema (um recorte do problema) e depois soluciona-lo através de um planejador externo, e executar a política encontrada para este planejador até se alcançar um estado fora deste recorte. Um novo recorte é criado a partir deste estado e o novo subproblema é resolvido pelo planejador e sua política é simulada novamente. Este procedimento é repetido até chegar a um estado meta (Trevizan, 2013). Os algoritmos que aplicam esta idéia serão apresentados na Seção 3.2 .3 . 
Nesta seção será definido esse subproblema de SSP MDPs e será descrito um algoritmo que gera um Short-Sighted SSP MDP. Cada subproblema criado tem as seguintes características:

- Os estados com probabilidade 0 (zero) de serem alcançados após a execução de $t$ ações são podados do subproblema;

- Um conjunto de estados meta artificiais é criado; e

- O custo de alcançar um estado meta artificial é incrementado por uma heurística que visa guiar a busca pelos estados meta do problema original.

Para entender como o problema é segmentado, primeiro é necessário observar que métrica será utilizada para realizar a poda no problema original. Esta métrica é definida por:

$$
\delta\left(s, s^{\prime}\right)= \begin{cases}0 & \text { se } s=s^{\prime} \\ 1+\min _{a \in A} \min _{\hat{s}: P(\hat{s} \mid s, a)>0} \delta\left(\hat{s}, s^{\prime}\right) & \text { caso contrário. }\end{cases}
$$

Em que $\delta\left(s, s^{\prime}\right)$ define o número mínimo de ações a serem executadas a partir do estado $s$ para se alcançar o estado $s^{\prime}$. Esta métrica pode ser considerada como a distância não simétrica "em ações" de $s$ a $s^{\prime}$.

\section{Definição 2.4.1. (Short-Sighted Stochastic Shortest Path MDP (Short-Sighted SSP MDP)) (Trevizan, 2013)}

Dado um SSP MDP definido pela tupla $\mathcal{S}=\left\{S, A, C, P, G, s_{0}\right\}$, é possivel ter um Short-Sighted $S S P M D P$ centrado em $s \in S$ e com profundidade $t \in N^{*}$ definido por $\mathcal{S}_{s, t}=\left\{S_{s, t}, A, C_{s, t}, P, G_{s, t}, s\right\}$, em que:

- $S_{s, t}$ é o conjunto de estados resultantes da poda, que podem ser alcançados a partir de $s$ executando até $t$ açôes. Este conjunto é definido por:

$$
S_{s, t}=\left\{s^{\prime} \in S \mid \delta\left(s, s^{\prime}\right) \leq t\right\}
$$

- $G_{s, t}$ é o conjunto de estados meta artificiais, que são os estados alcançados a partir de $s$ executando exatamente $t$ açôes. Este conjunto é definido por:

$$
G_{s, t}=\left\{s^{\prime} \in S \mid \delta\left(s, s^{\prime}\right)=t\right\} \cup\left(G \cap S_{s, t}\right)
$$

- $C_{s, t}\left(s^{\prime}, a, s^{\prime \prime}\right)$ é a função de custo de ir do estado s' ao estado s" executando a ação a do novo problema, definida por:

$$
C_{s, t}\left(s^{\prime}, a, s^{\prime \prime}\right)=\left\{\begin{array}{ll}
C\left(s^{\prime}, a, s^{\prime \prime}\right)+H\left(s^{\prime \prime}\right) & \text { se } s^{\prime \prime} \in G_{s, t} \backslash G \\
C\left(s^{\prime}, a, s^{\prime \prime}\right) & \text { caso contrário }
\end{array}, \forall s^{\prime} \in S_{s, t}, s^{\prime \prime} \in S_{s, t}, a \in A\right.
$$

em que $H(s)$ é uma heurística definida para o estado s. Caso não seja possivel, adotar um valor para esta heurística pode-se assumir $H(s)$ como 0 . Note que $G_{s, t} \backslash G$ é o conjunto de estados meta artificiais que não são metas reais do problema. Neste trabalho será considerado que o custo depende apenas de $s$ e a, i.e., $C_{s, t}(s, a)$ e $H\left(s^{\prime \prime}\right)=0$. 


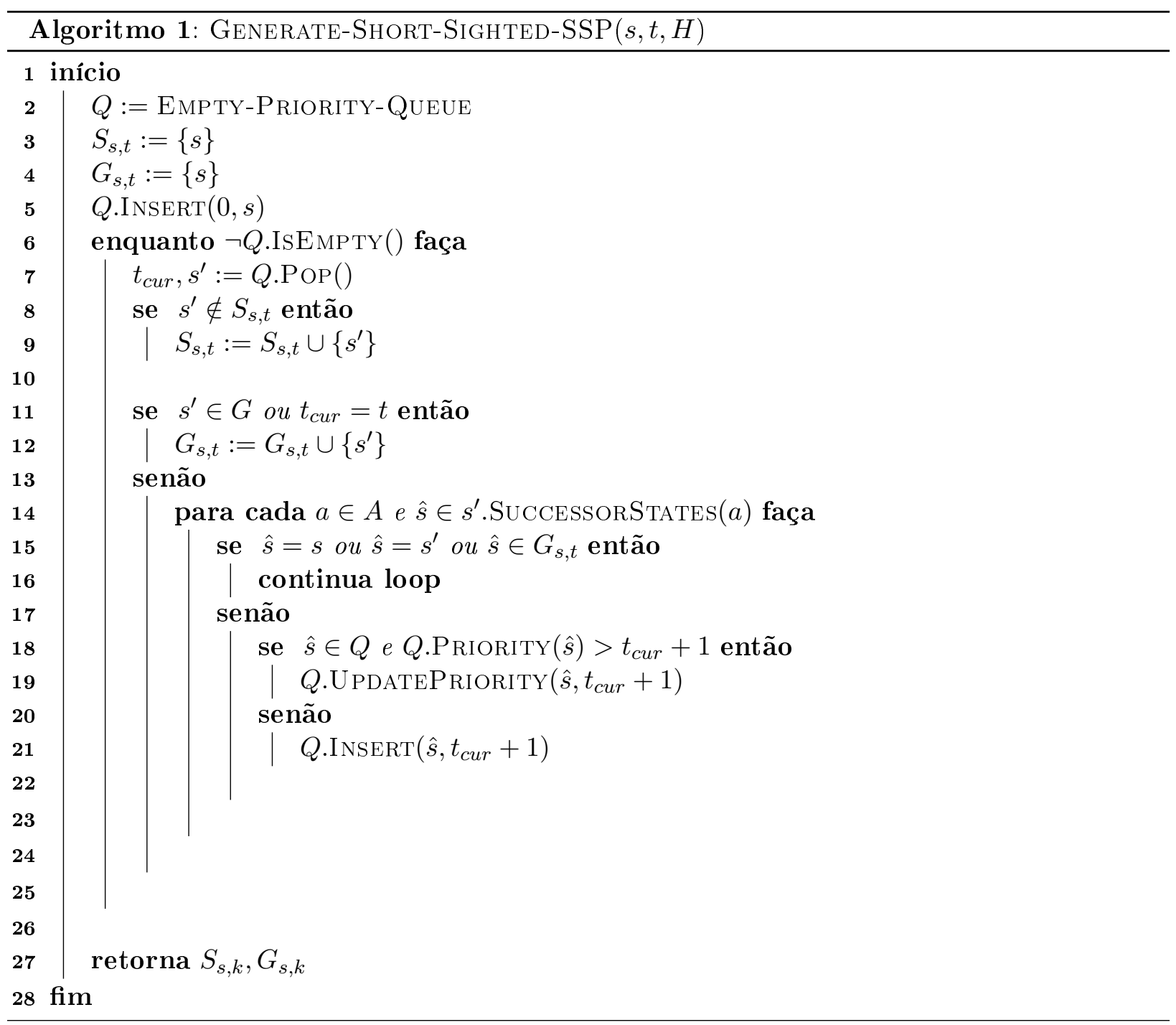

Uma maneira de gerar este Short-Sighted SSP MDP é realizar uma busca em largura no espaço de estados a partir do estado $s$ e interromper a busca à medida que ela alcança a profundidade $t$. O método Generate-Short-Sighted-SSP (Algoritmo 1) implementa esta busca, através do uso de uma fila de prioridades $Q$, em que a prioridade $t$ indica o número de ações necessário para se alcançar aquele estado. Os estados encontrados nesta busca são adicionados ao subconjunto $S_{s, t}$ (Algoritmo 1, Linha 8 e 9) e todos os estados encontrados na profundidade $t$ mais os estados meta encontrados nesta busca são inseridos no subconjunto de estados meta artificiais $G_{s, t}$ (Algoritmo 1, Linhas 11 a 12). Dado o estado $s^{\prime}$, todos os seus estados sucessores são identificados (Algoritmo 1, Linha 14) e marcados para visita. O estado sucessor $\hat{s}$ é inserido na fila de prioridade caso não tenha sido visitado antes (Algoritmo 1, Linha 21) ou sua prioridade é atualizada, caso já tenha sido marcado com uma prioridade menor (Algoritmo 1, Linhas 18 a 19). 


\section{Capítulo 3}

\section{Soluções de SSP MDPs}

Existem algoritmos para encontrar políticas ótimas para SSP MDPs baseados em programação dinâmica com garantias de convergência. Estes algoritmos podem realizar atualizações síncronas do valor do estado, i.e., atualizar todos os estados a cada iteração; ou realizar atualizações assíncronas (Bertsekas, 1982), que aplicam o Bellman update nos estados em uma ordem arbitrária, mantendo ainda suas propriedades de convergência sob certas condições.

Neste capítulo serão apresentadas as versões síncronas e assíncronas que servirão como base para os algoritmos propostos neste trabalho para resolver uma extensão de SSP MDPs.

\subsection{Soluções Síncronas para SSP MDPs}

A seguir são descritos dois algoritmos síncronos clássicos: Iteração de Valor (Howard, 1960) e Iteração de Política (Howard, 1960) para resolver SSP MDPs.

\subsubsection{Iteração de Valor para SSP MDPs}

O algoritmo de Iteração de Valor (IV, Algoritmo 2) (Howard, 1960) é uma solução clássica baseada em programação dinâmica síncrona que utiliza diretamente a equação de Bellman (Equação 2.6). O algoritmo inicializa a função valor com um valor arbitrário $V^{0}(s)$ para todo estado $s$ (Linha 3). A IV realiza atualizações de valores para todos os estados $s$, computando a próxima função valor $V^{t+1}(s)$ (Linha 7), baseado em $V^{t}$ aplicando o operador $B$ :

$$
V^{t+1}(s)=\left(B V^{t}\right)(s)=\min _{a \in A}\left\{Q^{t+1}(s, a)\right\},
$$

em que $Q^{t+1}(s, a)$ é o valor do estado $s$ quando aplicamos a ação $a$ e é dado por:

$$
Q^{t+1}(s, a)=C(s, a)+\sum_{s^{\prime} \in S} P\left(s^{\prime} \mid s, a\right) V^{t}\left(s^{\prime}\right)
$$

Esta atualização é conhecida como Bellman update.

A política gulosa $\pi(s)$ com relação a $V^{t}$ e o estado $s$ é definida da seguinte forma:

$$
\pi(s) \in \underset{a \in A}{\arg \min }\left\{C(s, a)+\sum_{s^{\prime} \in S} P\left(s^{\prime} \mid s, a\right) V^{t}\left(s^{\prime}\right)\right\} .
$$




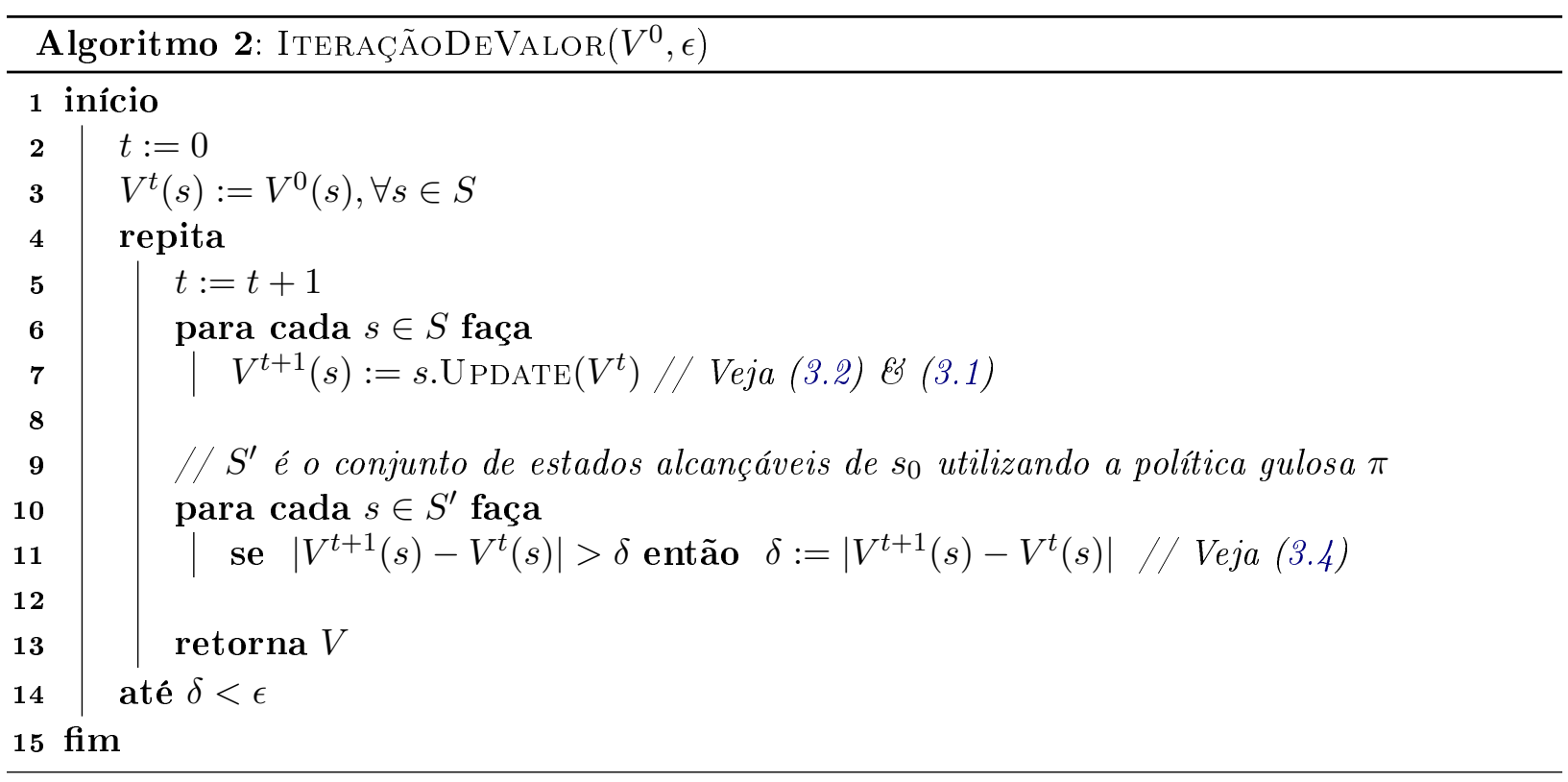

$V^{t}(s)$ converge para a única função valor ótima $V^{*}(s)$ em um limite infinito de atualizações, i.e., definindo $\epsilon_{t}=\max _{s}\left|V^{t}(s)-V^{*}(s)\right|$ temos $\lim _{t \rightarrow \infty} \epsilon_{t}=0$ (Bertsekas, 1982).

Neste trabalho, será considerado o conceito de $\epsilon$-otimalidade. Uma função valor é $\epsilon$-ótima se:

$$
B E=\max _{s \in S^{\prime}}|V(s)-(B V)(s)| \leq \epsilon,
$$

onde $S^{\prime}$ é o conjunto de estados alcançáveis a partir de $s_{0}$ quando se está seguindo a política gulosa $\pi$ com respeito a $V$. BE é conhecido como o resíduo de Bellman.

Neste algoritmo também deve-se considerar os conceitos de admissibilidade e monotonicidade da função valor. A função valor $V^{0}$ é admissível se e somente se $V^{0}(s) \leq V^{*}(s), \forall s \in S$. A função valor $V$ é monotônica se $V(s) \leq(B V)(s), \forall s \in S$.

O operador B preserva a admissibilidade, i.e., se $V(s) \leq V^{*}(s), \forall s \in S,\left(B^{k} V\right)(s) \leq V^{*}(s) \forall s \in S$ e $k \in N^{*}$; e preserva a monotonicidade $V(s) \leq\left(B^{k} V\right)(s), \forall s \in S$ e $k \in \mathbb{N}^{*}$ (Bertsekas e Tsitsiklis, 1996). Nestas definições $B^{k}$ é a composição do operador $B$, i.e., $\left(B^{k} V\right)(s)=\left(B\left(B^{k-1}\right) V\right)(s), \forall s \in S$.

\subsubsection{Iteração de Política para SSP MDPs}

Enquanto o algoritmo de Iteração de Valor (Howard, 1960) resolve um SSP MDP melhorando uma função valor arbitrária, o algoritmo de Iteração de Política (IP, Algoritmo 3) tenta melhorar uma política própria.

Dado uma política arbitrária $\pi_{0}$ (Linha 3), o algoritmo trabalha através da execução alternada de duas etapas até encontrar uma política ótima. A primeira etapa é chamada de avaliação de política em que a política é avaliada através do cálculo da função valor para cada estado (Linha 6). Isto é feito montando um sistema de equações utilizando uma versão simplificada da equação de Bellman para cada estado:

$$
V^{i}(s):=C(s, a)+\sum_{s^{\prime} \in S} P\left(s^{\prime} \mid s, \pi^{i}(s)\right) V^{i}\left(s^{\prime}\right) .
$$

É possível resolver esse sistema aplicando algum método já conhecido de álgebra linear, como o 


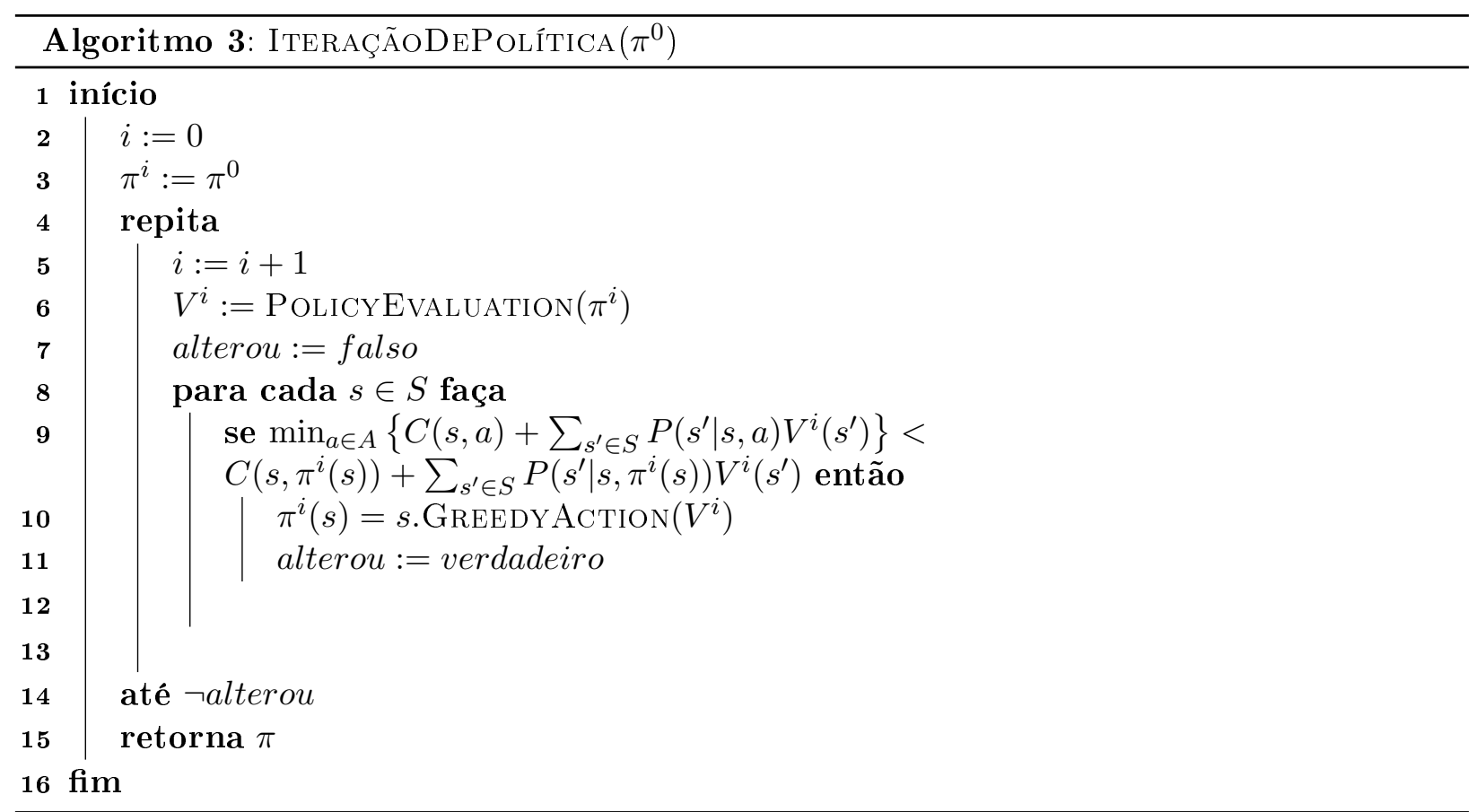

método de eliminação gaussiana (Cunha, 2000, p. 32).

Uma vez com a função valor calculada para cada estado, a segunda etapa, chamada de melhoria de política (Linhas 7 a 13), é executada, onde cada estado é avaliado com o intuito de se verificar se há alguma ação a que tenha um valor menor que o valor obtido por $\pi^{i}$, através da seguinte comparação:

$$
\min _{a \in A}\left\{C(s, a)+\sum_{s^{\prime} \in S} P\left(s^{\prime} \mid s, a\right) V^{i}\left(s^{\prime}\right)\right\}<C\left(s, \pi^{i}(s)\right)+\sum_{s^{\prime} \in S} P\left(s^{\prime} \mid s, \pi^{i}(s)\right) V^{i}\left(s^{\prime}\right) .
$$

Caso a avaliação indique que há alguma ação que retorna um valor melhor que o da política atual $\pi^{i}(s)$, a política é atualizada com o valor de $\pi^{i}(s)=\operatorname{s.GreEdy} \operatorname{ACtion}\left(V^{i}\right)$, definida por:

$$
\text { S.GreedyAction }(V):=\underset{a \in A}{\arg \min }\left\{C(s, a)+\sum_{s^{\prime} \in S} P\left(s^{\prime} \mid s, a\right) V\left(s^{\prime}\right)\right\} .
$$

\subsection{Programação Dinâmica Assíncrona para SSP MDPs}

Um dos primeiros algoritmos que se tornaram populares utilizando a programação dinâmica assíncrona foi o RTDP proposto por Barto et al. (1995) e com suas diversas derivações como o LRTDP (Bonet e Geffner, 2003), o BRTDP (McMahan et al., 2005), o FRTDP (Smith e Simmons, 2006) e o RTDP Bayesiano (Sanner et al., 2009).

Recentemente foram propostos os algoritmos SSiPP e LSSiPP (Trevizan, 2013) para resolver SSP MDPs. Esses algoritmos assíncronos segmentam o problema em diversos subproblemas (ShortSighted SSP MDPs, descritos na Seção 2.4), mais fáceis de serem resolvidos. 


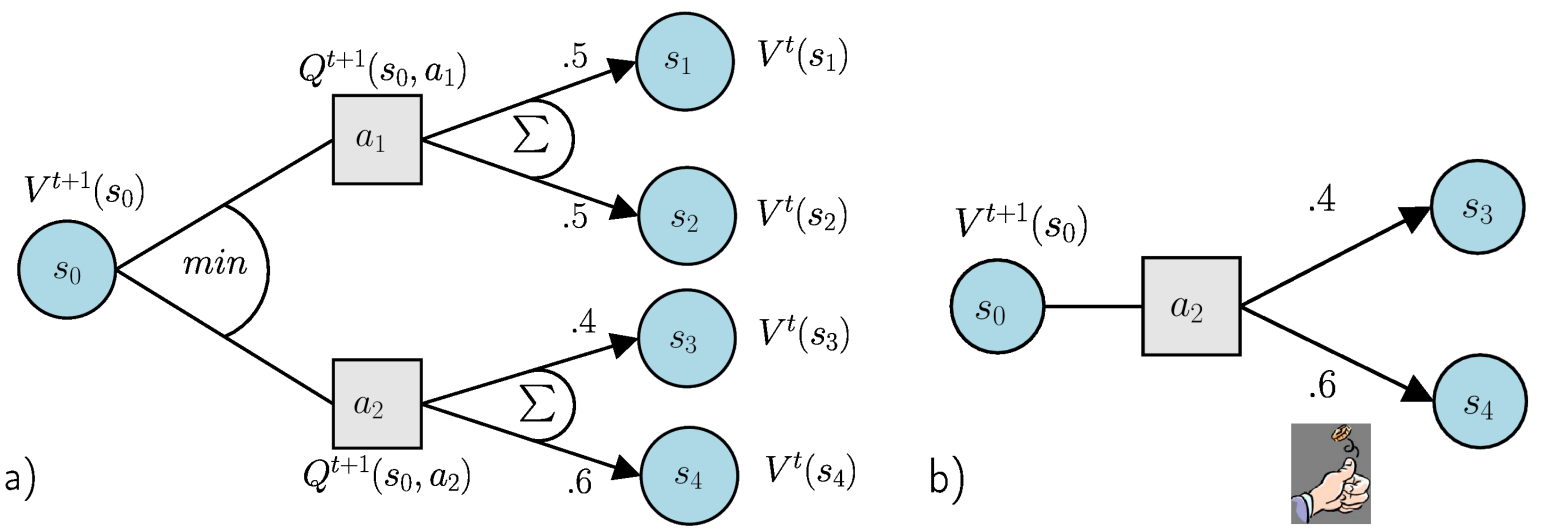

Figura 3.1: Operaçôes realizadas no algoritmo RTDP: a) Bellman update no RTDP e b) Escolha do próximo estado no RTDP.

\subsubsection{RTDP - Programação Dinâmica em Tempo Real}

O algoritmo de Programação Dinâmica em Tempo Real (Barto et al., 1995) (RTDP, do inglês Real-time Dynamic Programming) utiliza a idéia de simular a execução de uma política gulosa em um sistema para atualizar o valor dos estados via programação dinâmica. Para isto assume-se que o sistema se encontra em um estado inicial e a partir de cada simulação da execução de uma ação, cada estado visitado pela simulação é atualizado com um Bellman update (Figura 3.1a).

O algoritmo RTDP (Algoritmo 4) inicializa a função valor com uma função valor inicial (Algoritmo 4, linha 4). As simulações realizadas pelo algoritmo são chamadas de trials (Algoritmo 4, linhas 5 a 17) e visam explorar o espaço de estados a partir do estado inicial $s_{0}$, até alcançar um estado meta (Algoritmo 4, linha 8). A cada visita a um estado $s$, o algoritmo executa um Bellman update no estado (Algoritmo 4, linha 10) e realiza uma "simulação" da execução de uma ação a fim de visitar outro estado, escolhendo a ação gulosa $a$ e realizando um sorteio do próximo estado $s^{\prime}$ segundo as probabilidades de transição da ação (Algoritmo 4, linhas 11 e 12), exemplificado pela Figura 3.1b. Este sorteio pode ser descrito pela operação:

$$
\text { s.ChoosenextState }(a):=s^{\prime} \sim P(\cdot \mid s, a) .
$$

Definição 3.2.1. (Estados alcançáveis) Os estados que podem ser visitados por um agente durante a execução de qualquer política $\pi$ a partir de $s_{0}$ são chamados de estados alcançáveis (Barto et al., 1995).

Uma vez que é realizada uma busca por políticas parciais, i.e., políticas que envolvem somente estados alcançáveis de $s_{0}$, é importante se definir a idéia de estados relevantes.

Definição 3.2.2. (Estados relevantes) Dada uma política ótima $\pi^{*}$, os estados alcançáveis por $\pi^{*}$ são chamados de estados relevantes (Barto et al., 1995).

Assim, os estados relevantes são aqueles para o qual é necessário garantir a convergência para valores ótimos. A convergência do RTDP é garantida se todos os estados são inicializados com uma função valor $V^{0}$ admissível.

Ao finalizar um trial, o RTDP realiza uma atualização da função valor para os estados visitados (Linhas 14 a 16) a fim de refinar a função valor para estes estados.

Uma explicação detalhada da convergência do RTDP será mostrada a seguir. 


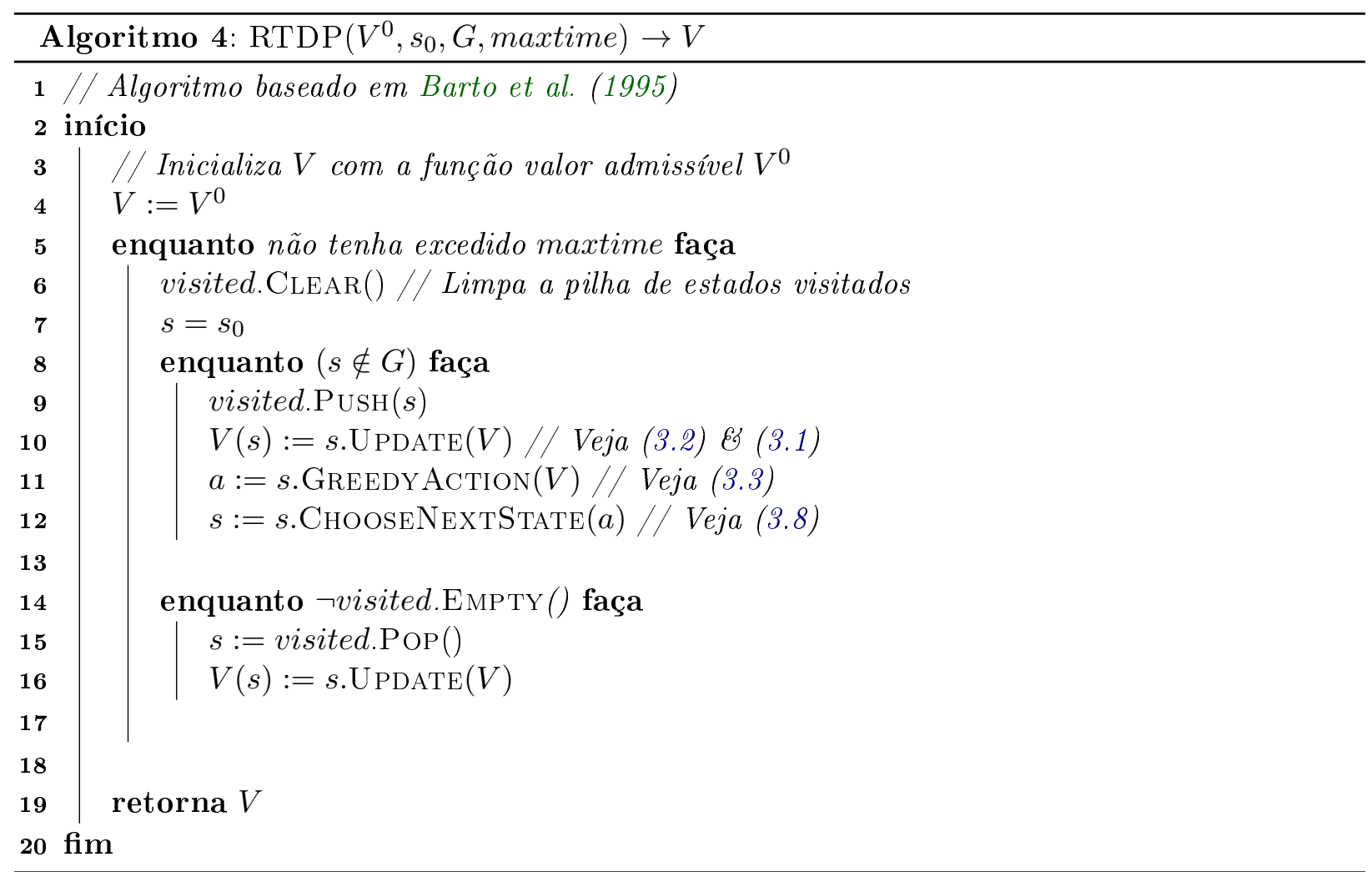

\section{Convergência do RTDP para SSP MDPs}

Nesta seção é fornecida uma idéia geral da prova de convergência do RTDP apresentada por Barto et al. (1995) pois o mesma será utilizada como base para a prova de convergência do algoritmo apresentado neste trabalho no Capítulo 6 para SSP MDP-IPs.

Uma vez que RTDP é um caso especial do algoritmo de programação dinâmica assíncrona, a sua prova de convergência é construída com base na prova de convergência da programação dinâmica assíncrona.

Definição 3.2.3. (Programação Dinâmica Assíncrona (Sutton e Barto, 1998)) Na programação dinâmica assíncrona, as atualizações da função valor não precisam ser executadas de uma maneira sistemática. Os estados são atualizados em qualquer ordem utilizando valores de outros estados que estejam disponiveis. Antes de atualizar o valor de alguns estados, outros estados podem ter sido atualizados diversas vezes.

Esta definição é genérica e não assume um operador de atualização da função valor em particular e nem uma função valor inicial. Bertsekas (1982) prova que a condição necessária para a convergência da programação dinâmica assíncrona é que o operador de atualização deve ter a propriedade de contração com respeito a norma do supremo (sup-norm) (Bertsekas, 1982).

Teorema 3.2.4. A programação dinâmica assíncrona para SSP MDPs converge para o valor ótimo se o valor de cada estado é atualizado infinitas vezes (Bertsekas, 1982).

A chave para a prova de convergência do Teorema 3.2.4 é que o operador $B$, definido pela Equação 3.1, tem a propriedade de contração com respeito a norma do supremo (sup-norm) (Bertsekas, 1982). Outro ponto a ser notado é o fato que o RTDP é um caso especial de programação dinâmica assíncrona onde um único estado é atualizado em cada instante de tempo. Se o conjunto de 
estados iniciais for $I=S$, é possível garantir que todos os estados serão atualizados infinitas vezes (Barto et al., 1995). Porém, se $I=\left\{s_{0}\right\}$ ou qualquer $I \subset S$, em que somente um subconjunto de $S$ pode ser alcançado a partir do conjunto de estados iniciais, não é possível garantir que todos os estados serão atualizados infinitas vezes e assim o Teorema 3.2.4 não pode ser usado diretamente para provar a convergência do RTDP.

Para se provar a convergência do RTDP com $I \subset S$ é preciso mostrar que apenas são necessários operações de programação dinâmica assíncrona sobre o conjunto de estados relevantes (Definição 3.2.2). O Teorema 3.2.5 garante que para um SSP MDP com uma função valor inicial admissível, o RTDP converge para os estados relevantes.

Teorema 3.2.5. (Convergência do RTDP) Em um SSP MDP, se a função valor inicial $V^{0}$ for admissivel, repetidos trials do RTDP alcançarão eventualmente valores ótimos sob todos os estados relevantes (Bonet e Geffner, 2003).

A chave para a prova deste teorema se deve ao fato de o operador $B$ preservar a admissibilidade durante os trials do RTDP, o que fará que o RTDP escolha a política ótima.

\subsubsection{LRTDP - Programação Dinâmica em Tempo Real Rotulada}

A convergência do RTDP pode ser lenta, pois estados com probabilidade baixa serão visitados menos frequentemente e seus sucessores não serão atualizados muito.

Pensando em como aprimorar o tempo de convergência do RTDP, o algoritmo Programação Dinâmica em Tempo Real Rotulada (LRTDP, do inglês Labeled Real-time Dynamic Programming), foi proposto por Bonet e Geffner (2003).

O LRTDP identifica os estados convergidos durante os trials. Estes estados $s$ são rotulados como "resolvidos" verificando se $V^{t}(s)$ tem valor ótimo. Desta forma, o trial que alcança um estado convergido termina antes, evitando assim atualizações desnecessárias nos estados resolvidos.

O algoritmo LRTDP (Algoritmo 5) inicializa $V$ com uma função valor admissível e realiza uma série de simulações (trials) semelhantes ao RTDP, exceto por sua condição de parada. Nestes trials, a condição de parada é acrescida de uma verificação de se o estado atual visitado já foi resolvido (Algoritmo 5, linha 8). Caso o estado tenha sido resolvido, o trial será interrompido. No final de cada trial uma chamada do algoritmo CheckSolved (Algoritmo 6) é realizada para cada estado visitado no trial em ordem reversa, com o intuito de verificar se algum destes estados pode ser rotulado como resolvido (Algoritmo 5, linhas 16 a 21).

O algoritmo CheckSolved (Algoritmo 6) rotula cada estado $s$ como resolvido quando todos os estados alcançáveis a partir de $s$ com a política gulosa estiverem resolvidos. O grafo enraizado no estado $s$ e constituído por estados alcançáveis a partir de $s$ é chamado de grafo guloso e o conjunto de estados pertencentes ao grafo é chamado de envelope guloso. Este algoritmo realiza uma busca em largura neste grafo procurando por algum estado em que $s \cdot \operatorname{ReSiduAL}() \geq \epsilon$, onde $s \cdot \operatorname{Residuat}()=\left|V^{t+1}(s)-V^{t}(s)\right|$ (Algoritmo 6, linha 10). Caso esse estado seja encontrado, seus sucessores não serão visitados (o que indica que o grafo guloso a partir de $s$ não foi resolvido, portanto os estados deste grafo ainda não serão rotulados). Para controlar os estados visitados, o algoritmo mantém um pilha com os estados "encerrados" (closed) e uma pilha com os estados "abertos" (open), que serão considerados na busca. Se existirem mais estados $s \operatorname{com} s \cdot \operatorname{ReSiduAL}() \geq \epsilon$ no grafo, estes estados são atualizados (Algoritmo 6, linhas 26 a 29), ao atualizar esses estados o LRTDP acelera 


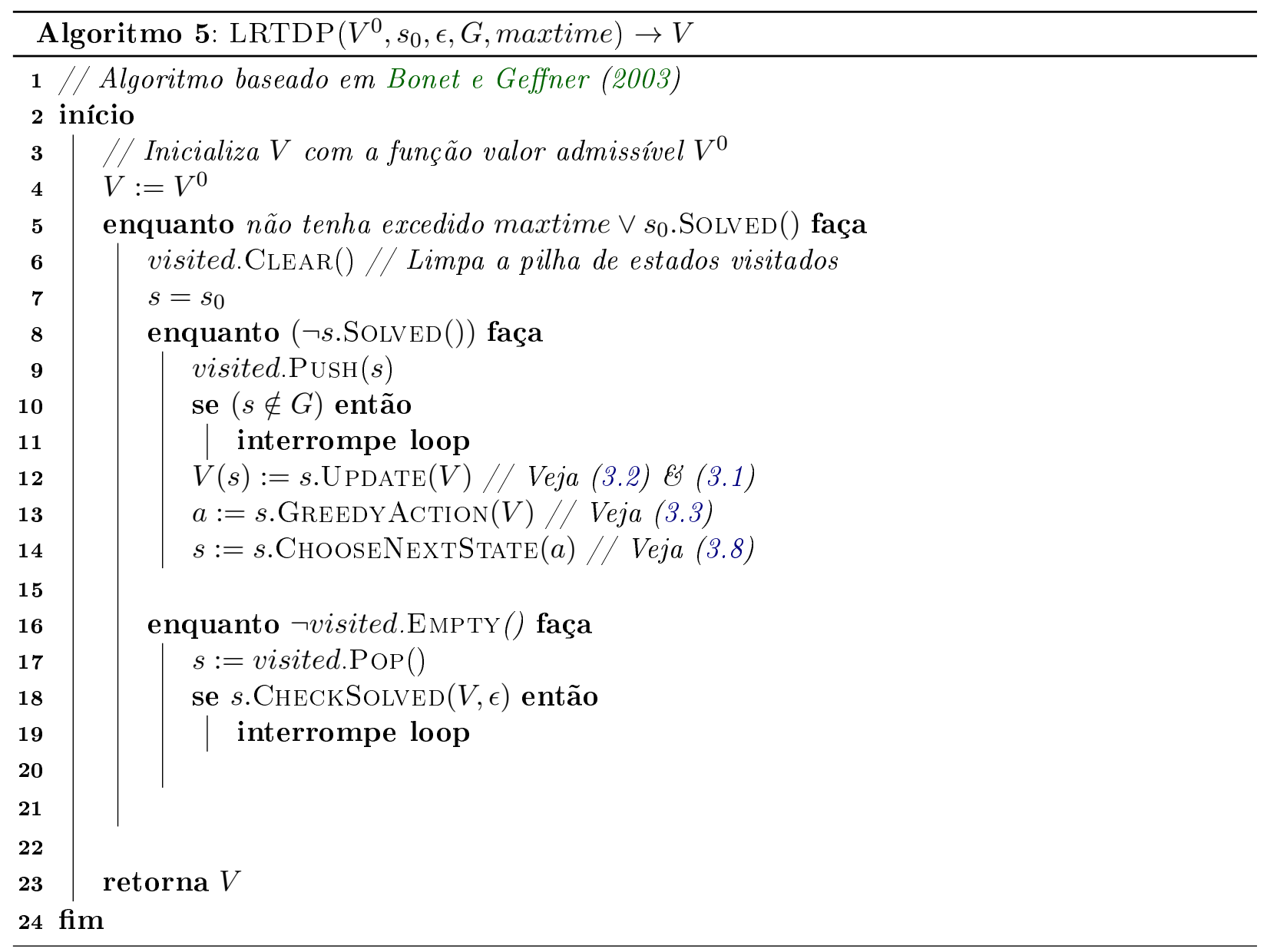

também a convergência. Caso não existam nenhum estado $s \operatorname{com} s \cdot \operatorname{ReSiduAL}() \geq \epsilon$, todos os estados da pilha closed ("encerrados") serão marcados como resolvidos (Algoritmo 6, linhas 21 a 24).

Ao encerrar um trial quando um estado resolvido tem sido alcançado, esses trials são reduzidos e os estados não resolvidos serão visitados mais frequentemente.

\subsubsection{SSiPP - Planejador Probabilístico Míope}

Um planejador simples que resolve um SSP MDP criando subproblemas (Short Sighted SSP MDPs) é o Non-Learning-Planner (Algoritmo 7) (Trevizan, 2013). Dado um estado inicial $s$ e uma profundidade $t$, o algoritmo cria um Short-Sighted SSP MDP centrado em $s$ através do método Generate-Short-Sighted-SSP (Algoritmo 1 chamado na Linha 4 do Algoritmo 7) e resolve o subproblema utilizando um algoritmo qualquer que resolva SSP MDPs no método SSP-Solver (Algoritmo 7, Linha 5). Uma vez resolvido, o algoritmo executa a política encontrada (através do método ApplyAction, que simula a execução de uma ação no SSP MDP) até encontrar um estado-meta artificial $s \in G_{s, t}$ (Algoritmo 7, Linhas 6 e 7). Caso $s$ seja um estado meta do SSP MDP o planejador é encerrado com sucesso, caso contrário, ele novamente cria um Short-Sighted SSP MDP agora centrado no novo estado $s$ e repete o ciclo (Algoritmo 7, Linhas 3 a 7) até alcançar um estado meta do problema original. Um exemplo desta execução é encontrado na Figura 3.2, em que a partir do estado inicial um Short-Sighted SSP MDP (representada pela nuvem) é criado, solucionado e simulado. Ao se chegar em um estado na profundidade $t$, na borda do conjunto, um novo Short-Sighted SSP MDP será criado e resolvido novamente, repetindo este ciclo até chegar a 

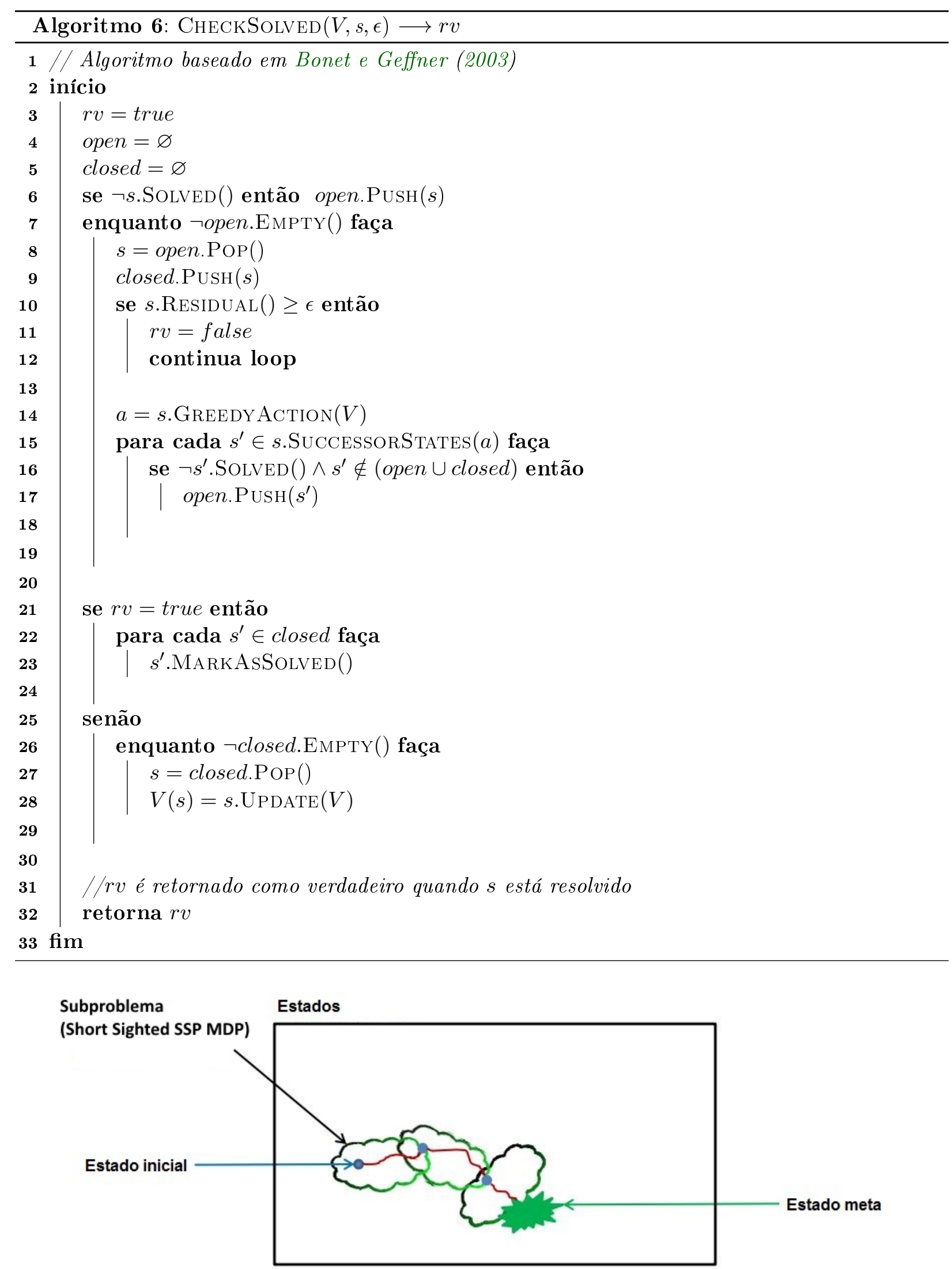

Figura 3.2: Exemplo de uma execução do planejador Non-Learning-Planner, baseado em Trevizan (2013).

um estado meta do problema (representado pelo conjunto marcado em verde na figura). 

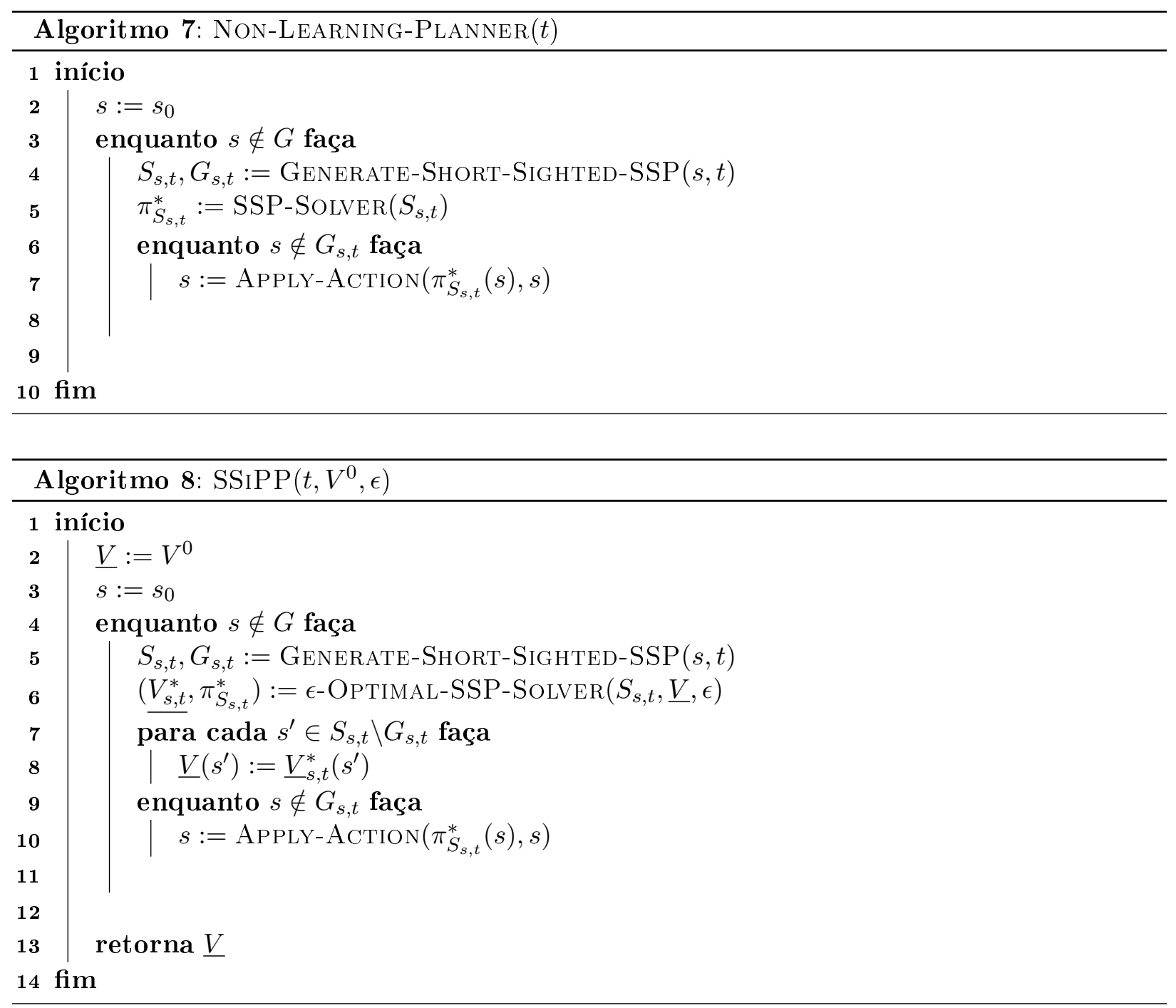

Porém, ao se solucionar um problema de planejamento utilizando um Short-Sighted SSP MDP é importante notar que o comportamento do algoritmo Non-LEARning-Planner depende da escolha do planejador externo usado no método SSP-SOLVER, podendo não encontrar a solução ótima do SSP MDP (Trevizan, 2013). Para corrigir este problema é necessário pensar em alguma forma de atualizar a função valor, chamada de $\underline{V}$, um limite inferior a $V^{*}$, e enviá-la ao planejador externo, de tal maneira que ele aprenda melhor por quais caminhos deve ir.

O Planejador Probabilístico Míope (SSiPP, do inglês Short-Sighted Probabilistic Planner) (Algoritmo 8) inicializa a estimativa de $\underline{V}$ com um valor admissível $V^{0}$ (Algoritmo 8, linha 2). Ao resolver um Short-Sighted SSP MDP envia o valor $\underline{V}$ para o planejador escolhido para resolver o subproblema (Algoritmo 8, linha 6) e utiliza o valor de $V_{S_{s, t}}^{*}$ encontrado na resolução do subproblema para atualizar $\underline{V}$, excluindo desta atualização os estados-meta artificiais do subproblema (Algoritmo 8, linhas 7 e 8).

Assim, a cada resolução de um subproblema o SSiPP melhora o valor de $\underline{V}$, fazendo-o se aproximar mais de $V^{*}$. Para que isto seja possível, o planejador externo que soluciona os Short-Sighted SSP MDPs gerados precisa ser $\epsilon$-ótimo, ou seja, ele deve encontrar a solução ótima com um erro menor ou igual a $\epsilon$. Desta forma, ao se executar o SSiPP um número considerável de vezes o valor de $\underline{V}$ convergirá para $V^{*}$ para todo o problema (Trevizan, 2013). O Algoritmo 9 executa o SSiPP 


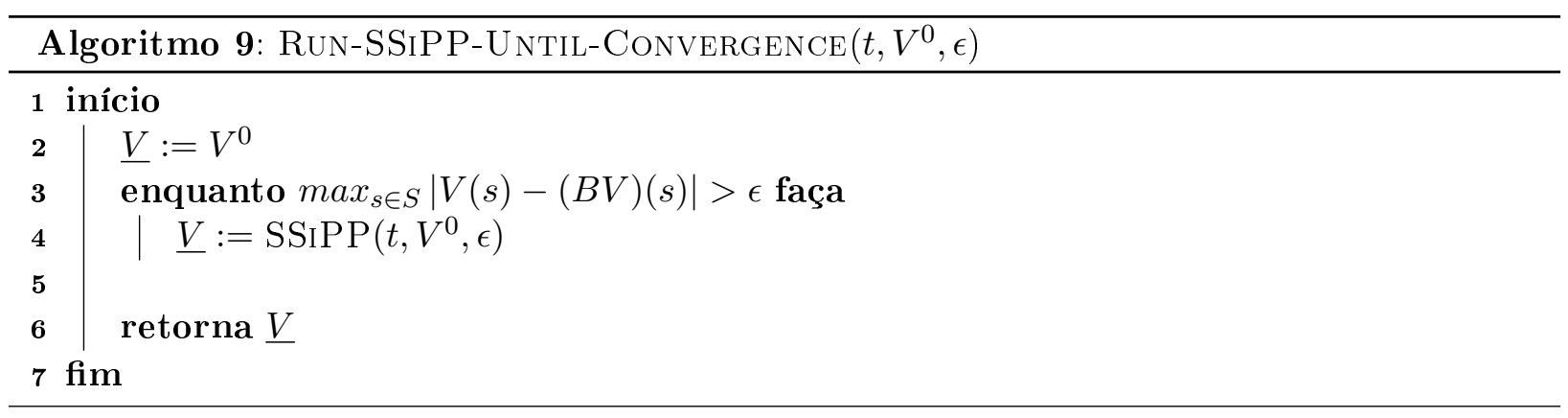

várias vezes, considerando um resíduo $\max _{s \in S}|V(s)-(B V)(s)|>\epsilon$.

Um possível planejador $\epsilon$-ótimo que pode ser utilizado em conjunto com o SSiPP é o RTDP.

\subsubsection{LSSiPP - Planejador Probabilístico Míope Rotulado}

Ao tentar resolver um SSP MDP com o SSiPP, o SSiPP sofre dos mesmos problemas que o RTDP, i.e., por não saber quais estados convergiram, o algoritmo pode acabar criando um ShortSighted SSP MDP e tentar resolve-lo a partir de um grupo de estados que já convergiu previamente, fazendo mais atualizações em $\underline{V}$ que não seriam necessárias. Uma forma de evitar estas atualizações é utilizar os mesmos mecanismos de rotulamento de estados já convergidos que o LRTDP utiliza. Esta versão do algoritmo é chamada de Labeled SSiPP ou LSSiPP (Algoritmo 10).

O algoritmo verifica se o estado visitado está resolvido na condição de parada do algoritmo (Algorimo 10, Linha 4). No momento de gerar um Short-Sighted SSP MDP adiciona os estados rotulados no conjunto de estados meta artificiais (Algoritmo 10, Linhas 5 a 9) e faz uma verificação dos estados convergidos através do CHECKSolved (Algoritmo 6) ao final da execução do LSSiPP (Algoritmo 10, Linhas 19 a 22).

\subsection{Soluções para SSP MDP Fatorados}

Nesta seção serão brevemente apresentados alguns algoritmos síncronos e assíncronos para resolver SSP MDPs Fatorados. Eles usam Diagramas de Decisão Algébricas (ADDs, do inglês Algebraic Decision Diagrams) (Bahar et al., 1993) para representar e resolver eficientemente este tipo de problemas.

\subsubsection{Diagramas de Decisão Algébricos.}

Os diagramas de decisão algébricos (ADDs) (Bahar et al., 1993) são usados para representar de forma compacta funções $f:\{0,1\}^{n} \rightarrow \mathbb{R}$ com independência específica de contexto (Boutilier et al., 1996) e estrutura compartilhada. Os ADDs são extensões dos BDDs (diagramas de decisão booleanos, do inglês Boolean Decision Diagrams) (Bryant, 1992) que representam funções booleanas $f:\{0,1\}^{n} \rightarrow\{0,1\}$. Os ADDs são grafos dirigidos acíclicos (DAGs, do inglês directed acyclic graphs) onde a variável de teste $Y_{i} \in Y(1 \leq i \leq n)$ em qualquer caminho da raiz à folha segue uma ordenação de variáveis total e fixa $\mathbb{O}$. Em um ADD, subgrafos idênticos são mesclados, o que pode resultar em uma representação compacta de $f$. Por exemplo, a função de custo $C:\{0,1\}^{n} \rightarrow \mathbb{R}$ representada na Figura 3.3a como uma árvore de decisão pode ser representação como uma ADD em uma forma compacta (Figura 3.3b). 


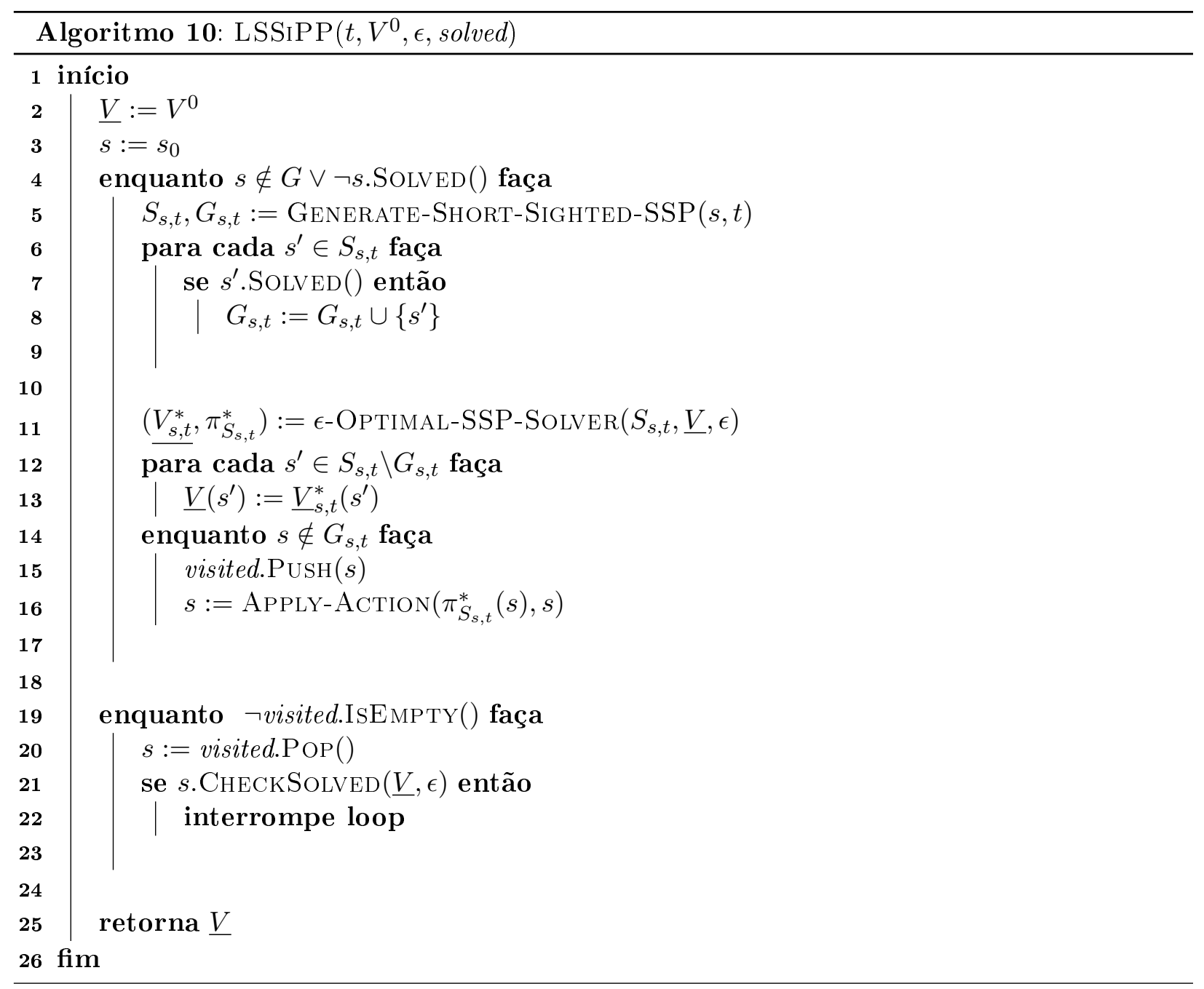

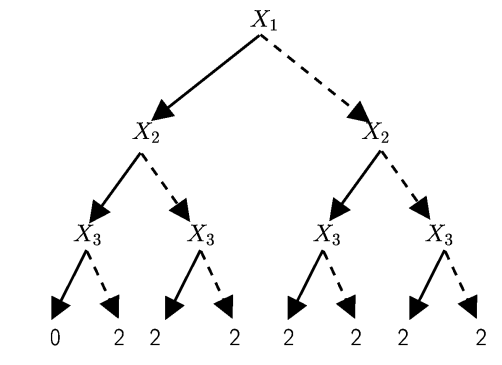

a)

Figura 3.3: Um exemplo de função custo representada como a) uma árvore e b) como um ADD. A linha contínua representa a ramificação verdadeira (1) do teste de uma variável e a linha segmentada representa a ramificação falsa (0). O custo é 0 para $\vec{x}=\left\{X_{1}=1, X_{2}=1, X_{3}=1\right\}$, e 2 para os outros casos.

Dada uma função booleana $f$ representada como um ADD, chamada $F$, um conjunto de variáveis $Y$ com a atribuição das variáveis $\vec{y} \in\{0,1\}^{n}, \operatorname{Eval} A D D(F, \vec{y})$ retorna o valor real da folha seguindo o caminho correspondente a partir da raiz do ADD.

Operações unárias como min, max, marginalização sobre variáveis $\left(\sum_{x_{i} \in X_{i}}\right)$, bem como operações binárias como a adição $(\oplus)$, subtração $(\ominus)$, multiplicação $(\otimes), \min (\cdot, \cdot)$ e $\max (\cdot, \cdot)$ podem ser realizadas eficientemente em um ADD. 


\subsubsection{Algoritmos Síncronos e Assíncronos para SSP MDP Fatorados}

O algoritmo SPUDD (do inglês Stochastic Planning using Decision Diagrams)(Hoey et al., 1999) é um algoritmo síncrono eficiente que resolve MDPs fatorados. Ele estende o algoritmo de Iteração de Valor usando os Diagramas de Decisão Algébricas (ADDs) para representar de forma compacta as funções de transição e custo, e também para calcular de forma eficiente a política ótima.

Existem também alguns trabalhos na literatura sobre soluções assíncronas fatoradas para SSP MDPs: sRTDP (Feng et al., 2003), LAO* simbólico (Feng e et al., 2002), factRTDP(Holguin, 2013), factLRTDP (Holguin, 2013) and factBRTDP (Delgado et al., 2010). Enquanto o sRTDP (Feng et al., 2003) atualiza um estado abstrato (um grupo de estados similares), o factRTDP e o factLRTDP (Holguin, 2013) atualizam um único estado em um conjunto de simulações baseadas em trials. O algoritmo factBRTDP é a versão fatorada do Bounded RTDP (McMahan et al., 2005) e não é tão eficiente quanto o factLRTDP visto que é muito custoso atualizar o limite superior e inferior de uma função valor. Os algoritmos fatorados que são propostos neste trabalho são baseados no factRTDP e factLRTDP (Holguin, 2013). 


\section{Capítulo 4}

\section{Processos de Decisão Markovianos com Probabilidades Imprecisas}

Como foi observado nos exemplos 1 e 2 mostrados no Capítulo 1, muitas vezes em problemas reais é necessário trabalhar com probabilidades imprecisas a fim de lidar com informações imprecisas ou conflitantes, dados insuficientes, probabilidades não estacionárias e a ocorrência de eventos imprevisíveis.

Os Processos de Decisão Markovianos com Probabilidades Imprecisas (MDP-IPs, do inglês Markov Decision Processes with Imprecise Probabilities), foram propostos por Satia e Lave Jr. (1970), para lidar com estas situações. Neste capítulo será definido formalmente uma classe mais geral de MDP-IPs, chamados SSP MDP-IPs e será descrito o algoritmo Iteração de Valor para SSP MDPIPs. Também serão descritos brevemente outros arcabouços que podem ser utilizados para lidar com as imprecisões nas probabilidades de transição, além dos SSP MDP-IPs.

\subsection{SSP MDP-IPs - MDP-IPs de Horizonte Indeterminado}

Um SSP MDP-IP é um processo de decisão sequencial com estados, ações, custo, estado inicial e estados meta semelhante a um SSP MDP, exceto pelo fato de sua função de transição probabilística ser definida com imprecisão. Nesta extensão de um SSP MDP, ao invés de haver uma medida de probabilidade $P(\cdot \mid s, a)$ sob um espaço de estados $S$ para as transições, há um conjunto de medidas de probabilidades.

Por exemplo, suponha um SSP MDP-IP com quatro estados $s_{0}, s_{1}, s_{2}$ e $s_{3}$ e duas ações $a_{1}$ e $a_{2}$. A Figura 4.1 mostra a função de transição probabilística para o estado $s_{0}$ e a ação $a_{1}$ com três possíveis próximos estados $s_{1}, s_{2}$ e $s_{3}$, além de mostrar as probabilidades de transição dadas pelos parâmetros $p_{1}, p_{2}$ e $p_{3}$, i.e., $p_{1}=P\left(s_{1} \mid s_{0}, a_{1}\right), p_{2}=P\left(s_{2} \mid s_{0}, a_{1}\right)$ e $p_{3}=P\left(s_{3} \mid s_{0}, a_{1}\right)$ (Figura 4.1a). $P\left(\cdot \mid s_{0}, a_{1}\right)$ é denotado para todo o conjunto de valores de probabilidades de transição, i.e., o conjunto de valores particulares para $p_{1}, p_{2}$ e $p_{3}$, com $p_{1}+p_{2}+p_{3}=1$. Outros parâmetros e/ou probabilidades também podem ser definidos para as transições aplicando $a_{1}$ aos estados $s_{1}, s_{2}$ e $s_{3}$, como pode ser visto na Figura $4.1 \mathrm{~b}$, onde é mostrada a matriz de transição completa para a ação $a_{1}$ que inclui os parâmetros $p_{i}$, com $i \leq 5$, todos sujeitos aos seguintes conjuntos de restrições: $\varphi_{1}=\left\{p_{1} \leq 2 / 3 ; p_{3} \leq 2 / 3 ; 2 * p_{1} \geq p_{2} ; p_{1}+p_{2}+p_{3}=1 ;\right\}$ e $\varphi_{2}=\left\{p_{4}+p_{5}=1\right\}$.

Analogamente, é possível definir a função de transição para a ação $a_{2}$, que pode incluir outros 


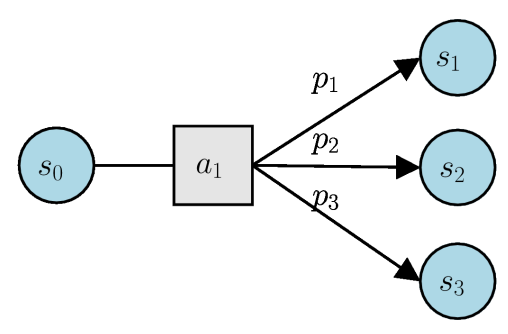

a)

\begin{tabular}{l|cccc}
$a_{1}$ & $s_{0}^{\prime}$ & $s_{1}^{\prime}$ & $s_{2}^{\prime}$ & $s_{3}^{\prime}$ \\
\hline$s_{0}$ & 0 & $p_{1}$ & $p_{2}$ & $p_{3}$ \\
$s_{1}$ & 0 & 0.5 & 0.3 & 0.2 \\
$s_{2}$ & 0 & 0 & 0.6 & 0.4 \\
$s_{3}$ & $p_{4}$ & 0 & $p_{5}$ & 0 \\
Restrições $\varphi_{1}:$ & & \multicolumn{2}{l}{ Restrições $\varphi_{2}:$} \\
$p_{1} \leq 2 / 3$ \\
$p_{3} \leq 2 / 3$
\end{tabular}

b)

Figura 4.1: Exemplo de um uma função de transição probabilística parametrizada de um SSP MDP-IP. a) Ilustração de $P\left(\cdot \mid s_{0}, a_{1}\right)$ b) Matriz de transição parametrizada completa para a ação $a_{1}$ e o conjunto de restrições sob os parâmetros, em que $\varphi_{1}$ está relacionado a $P\left(\cdot \mid s_{0}, a_{1}\right)$ e $\varphi_{2}$ está relacionado a $P\left(\cdot \mid s_{3}, a_{1}\right)$.

parâmetros sujeitos a um outro conjunto de restrições. O conjunto de restrições é chamado de $\varphi$, o vetor contendo o valor de cada parâmetro que satisfazem as restrições de probabilidades é representado por $\vec{p}$ e o conjunto de todas as medidas de probabilidade que satisfazem estas restrições é chamado de conjunto credal de transição.

Definição 4.1.1. (Conjunto credal de transição) Um conjunto credal contendo transiçôes condicionais sob o próximo estado $s^{\prime}$, dado o estado s e uma ação a é chamado de conjunto credal de transição (Cozman, 2000) e denotado como $K(\cdot \mid s, a)$. Assim, pode-se dizer que $P(\cdot \mid s, a) \in K(\cdot \mid s, a)$ define um conjunto de probabilidades de transição especificados de maneira imprecisa.

Assim como em (Buffet e Aberdeen, 2006), este trabalho assume independência entre o par estado-ação, conforme o pressuposto a seguir.

Pressuposto 4.1.2. (Independência do par estado-ação) As distribuiçôes de probabilidade $P(\cdot \mid s, a)$ são independentes de um par estado-ação para outro.

Neste trabalho é assumido que todos os conjuntos credais são fechados e convexos, e que eles não dependem do estágio $t$, i.e., $K\left(s^{\prime} \mid s, a\right)$ é estacionário. Também é assumido que as probabilidades imprecisas de um SSP MDP-IP são dadas em termos de um conjunto de parâmetros de probabilidade $p_{i}$ sujeitos a um conjunto de restrições $\varphi$, onde $\forall P(\cdot \mid s, a) \in K(\cdot \mid s, a), P(\cdot \mid s, a)$ satisfaz $\varphi$. Nesta definição cada $P\left(s^{\prime} \mid s, a\right)$ está associado ou a uma constante entre 0 e 1 ou a um parâmetro $p_{i}$.

Definição 4.1.3. (Stochastic Shortest Path MDP com Probabilidades Imprecisas (SSP MDP-IP)) Um SSP MDP-IP é definido por $\left\langle S, A, C, \mathcal{K}, G, s_{0}\right\rangle$ onde $S, A, C, G$ e $s_{0}$ são definidos como qualquer $S S P M D P$ e $\mathcal{K}$ é um conjunto de conjuntos credais de transiçẫo, onde cada conjunto credal de transição $K$ é definido para um par de estado-ação, i.e., $|\mathcal{K}| \leq|\mathcal{K}|_{\max }=|S \times A|$. Assim como nos SSP MDPs, os Pressupostos 2.2.1 e 2.2.2 são válidos.

Existem diversos critérios de otimização que podem ser utilizados para definir o valor de uma política de um SSP MDP-IP. Neste trabalho será utilizado critério o minimax que é um critério razoável para ser utilizado quando se deseja obter políticas robustas (Satia e Lave Jr., 1970). Este critério pode ser visto como um jogo soma-zero de dois jogadores, onde o ganho de um jogador é 
exatamente equilibrado com a perda de outro jogador. O agente seleciona as políticas que minimizam o custo futuro do agente sobre o pressuposto que a Natureza é adversária, i.e., escolhe a probabilidade que maximiza o custo esperado do agente.

Patek e Bertsekas (Patek e Bertsekas, 1999) definem um Jogo do Caminho Mais Curto Estocástico (Stochastic Shortest Path Game, SSP game) e provaram que existe um único valor de equilíbrio e também que existem políticas estacionárias que alcançam este equilíbrio, caso as políticas para o minimizador sejam apropriadas. Devido o Pressuposto 4.1.2, um SSP MDP-IP pode ser visto como um jogo sequencial, assim existe um valor de equilíbrio para este SSP MDP-IP e uma política determinística e estacionária que atinge este equilíbrio. Essa política induz a uma função valor ótima que é o ponto-fixo de:

$$
V^{*}(s)=\min _{a \in A} \max _{P \in K}\left\{C(s, a)+\sum_{s^{\prime} \in S} P\left(s^{\prime} \mid s, a\right) V^{*}\left(s^{\prime}\right)\right\}, \forall s \in S,
$$

\subsection{Iteração de Valor: Solução Síncrona e Enumerativa para SSP MDP-IPs}

Um algoritmo popular para resolver SPP MDP-IPs enumerativos baseado em programação dinâmica é o algoritmo Iteração de Valor (IV), proposto por Satia e Lave Jr. (1970) para MDP-IPs e adaptado por (Patek e Bertsekas, 1999) para SSP MDP-IPs, que realiza Bellman updates para todos os estados $s$, computando a próxima função valor $V^{t+1}(s)$ aplicando o operador $T$ da seguinte forma:

$$
V^{t+1}(s)=\left(T V^{t}\right)(s)=\min _{a \in A}\left\{Q^{t+1}(s, a)\right\}
$$

em que,

$$
Q^{t+1}(s, a)=C(s, a)+\max _{P \in K} \sum_{s^{\prime} \in S} P\left(s^{\prime} \mid s, a\right) V^{t}\left(s^{\prime}\right) .
$$

As equações (4.2) e (4.3) otimizam a escolha da ação $a \in A$ considerando o pior caso da distribuição $P \in K$ que maximiza a futura função valor esperada. Note que esta solução funciona com uma representação do espaço de estados enumerativa e pode ser ineficiente, pois deve resolver um problema de otimização (chamando um solver de programação linear caso $\varphi$ seja um conjunto de restrições lineares, ou chamando um solver de programação não-linear caso contrário) para cada passo, o que pode ser computacionalmente custoso para espaços de estado grandes.

Teorema 4.2.1. (Convergência da Iteração de Valor para SSP MDP-IPs (Patek e Bertsekas, 1999)) Para SSP MDP-IPs, $V^{t}(s)$ converge para a única função valor de equilíbrio $V^{*}(s)$ em um limite infinito de atualizaçôes, i.e.:

$$
\lim _{t \rightarrow \infty} \max _{s}\left|V^{t}(s)-V^{*}(s)\right|=0
$$

Uma função valor $V$ é $\epsilon$-ótima se: 


$$
B E_{I P}=\max _{s \in S^{\prime}}|V(s)-(T V)(s)| \leq \epsilon
$$

em que $S^{\prime}$ é o conjunto de estados alcançáveis a partir de $s_{0}$ seguindo a política gulosa $\pi$ com respeito a $V$ e BE $E_{I P}$ é conhecido como o resíduo de Bellman para SSP MDP-IPs.

\subsection{Outros arcabouços com modelos de transição imprecisas}

Para lidar com as imprecisões nas probabilidades de transição, além dos SSP MDP-IPs existem outros arcabouços que podem ser utilizados. Entre elas, dois tipos particulares de MDP-IPs enumerativos, chamados de BMDP e MDP-ST, além das abordagens por Planejamento Contigente, Abordagem Possibilística e o Controle Robusto.

\subsubsection{BMDP - MDP Delimitado}

Um tipo particular de MDP-IP enumerativo com horizonte infinito (Satia e Lave Jr., 1970) é o MDP delimitado ou Bounded-parameter Markov Decision Process (BMDP) (Givan et al., 2000), em que as probabilidades imprecisas são especificadas por intervalos, por exemplo $\left\{0.3 \leq p_{j} \leq\right.$ $0.5\}$. Uma solução para estes BMDPs é o algoritmo Iteração de Valor por Intervalos (Givan et al., 2000) que pode encontrar uma política ótima (sob o pior modelo) sem necessitar de técnicas de otimização custosas. O algoritmo PiorModelo-BMDP (Algoritmo 11) ordena os próximos estados pela função valor de forma decrescente e tenta atribuir as maiores probabilidades para os estados com a maior função valor. Neste algoritmo, os estados sucessores de $s$ são ordenados pela função valor na chamada da função SortNextStates (Linha 2). Após isto, o algoritmo tenta encontrar um estado $s_{j}$ tal que, para todos os estados $s_{i} \operatorname{com} i<j, p\left(s_{i}^{\prime} \mid s, a\right)=p^{\max }\left(s_{i}^{\prime} \mid s, a\right)$ (Linha 7) e para $m>j, p\left(s_{m}^{\prime} \mid s, a\right)=p^{\min }\left(s_{m}^{\prime} \mid s, a\right)$ (Linha 13). Existem também soluções para SSP BMDPs que são extensões do RTDP (Buffet e Aberdeen, 2006) e LAO* (Cui et al., 2006, Yin et al., 2007).

Entretanto, apesar de que os algoritmos utilizados para resolver um BMDP enumerativo podem encontrar uma política ótima sem necessitar de técnicas de otimização custosas, eles tem algumas limitações: eles não conseguem lidar com restrições gerais ou com entradas fatoradas.

\subsubsection{MDP-ST - MDP com Transições Valoradas por Conjuntos}

Outra subclasse de MDP-IPs são os MDPs com transições valoradas por conjuntos (Markov Decision Process with Set-valued Transitions, MDP-ST) (Trevizan et al., 2007), em que as distribuições de probabilidades são dadas sobre conjuntos finitos de estados. Neste modelo existem duas variedades de incertezas: a seleção probabilística de um conjunto alcançável e a escolha nãodeterminística de um estado sucessor a partir de um conjunto alcançável. Os algoritmos para resolver este problema também podem encontrar uma política ótima sem necessitar de técnicas de otimização custosas visto que o princípio de Bellman para MDP-STs pode ser simplificado (Trevizan et al., 2007). 

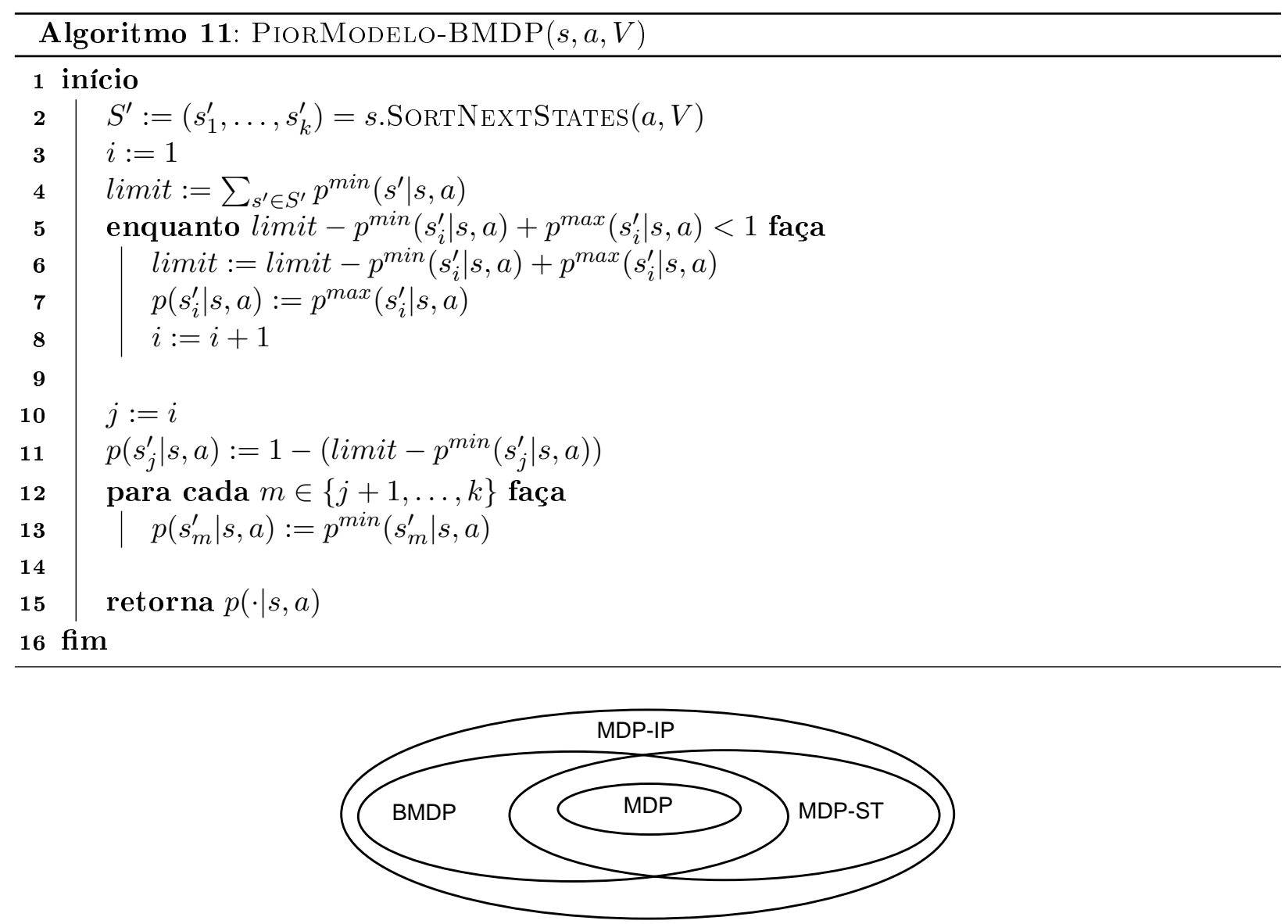

Figura 4.2: Relacionamento entre o MDP-IP e suas subclasses (Trevizan et al., 2007).

\subsubsection{Relação entre MDP, BMDP, MDP-ST e MDP-IP}

Na Figura 4.2, o relacionamento entre MDP, BMDP, MDP-ST e MDP-IP é mostrado. O BMDP e o MDP-ST não tem o mesmo poder de representação, i.e., alguns problemas descritos como MDP-ST não podem ser reduzidos a um BMDP e vice-versa. Visto que BMDPs e MDP-STs são casos especiais de MDP-IPs, um problema de qualquer dos dois tipos pode ser representado como um MDP-IP. Assim, os algoritmos para MDP-IPs claramente podem ser aplicados em BMDPs e MDP-STs (Trevizan et al., 2007).

\subsubsection{Planejamento Contingente e Abordagens Possibilísticas}

Os arcabouços alternativos que consideram os modelos de transição imprecisos como os MDP-IPs são o Planejamento Contingente (Contingent planning) (Hoffmann e Brafman, 2005) e as abordagens possibilísticas (Drougard et al., 2013, Sabbadin, 1999). Entretanto ambos os modelos não são probabilísticos. O Planejamento Contingente (Hoffmann e Brafman, 2005) é a tarefa de gerar um plano condicional dada a incerteza sobre o estado inicial e sobre os efeitos não-determinísticos das ações, porém com a habilidade de identificar alguns aspectos do estado inicial. As abordagens possibilísticas (Drougard et al., 2013, Sabbadin, 1999) têm uma visão mais forte sobre a incerteza das transições que somente possíveis transições (apesar de suas probabilidades relativas serem desconhecidas). Ambos podem ser formalizados como um MDP-IP com somente restrições de probabilidade 
entre $[0,1]$ nas transições probabilísticas, assim os algoritmos aplicados neste trabalho poderiam ser utilizados em tais traduções. Porém, este trabalho tenta lidar com o caso mais geral de restrições nas probabilidades de transição que somente $[0,1]$.

\subsubsection{Controle Robusto}

A área de Controle Robusto se preocupa em modelar um conjunto de probabilidades capazes de expressar a incerteza das transições de um determinado modelo de decisão, visto que pode ser difícil obter uma medida estatística acurada destas transições do sistema. Os trabalhos de Nilim e El Ghaoui (2005), Iyengar (2004) e Wiesemann et al. (2013) buscam principalmente modelar as imprecisões nas probabilidades dos problemas através de conjuntos de probabilidades obtidos por técnicas como regiões de verossimilhança e limites de entropia. Com a imprecisão uma vez modelada, o MDP é resolvido utilizando uma versão adaptada do algoritmo de programação dinâmica síncrona (equivalente ao algoritmo de Iteração de Valor para SSP MDP-IPs apresentado na Seção 4.2) que considera estes conjuntos através do critério maximin (para MDP-IPs que trabalham com recompensa) ou minimax (para MDP-IPs que trabalham com custo).

Outra abordagem de Controle Robusto utiliza a abordagem Bayesiana sobre os parâmetros $p_{i}$ de transição, onde é suposto que exista uma distribuição a priori sobre as probabilidades. Exemplos de aplicação desta abordagem podem ser vistas nos trabalhos de Delage e Mannor (2010) e Chen e Bowling (2012), que se focam em encontrar medidas de percentis que maximizem o valor a ser obtido dado um intervalo percentual de confiança. Estas abordagens são baseadas no conceito de risco, e tem sido chamadas de abordagem robusta "soft". 


\section{Capítulo 5}

\section{Processos de Decisão Markovianos com Probabilidades Imprecisas Fatorados}

Na prática um problema de planejamento probabilístico geralmente é descrito em termos de variáveis de estado. Para trabalhar com este modelo é preciso definir um modelo fatorado para os SSP MDP-IPs. Neste capítulo será apresentado este modelo, os SSP MDP-IPs fatorados, bem como o procedimento para gerar um SSP MDP-IP enumerativo a partir de um modelo fatorado. Também será mostrado o algoritmo Iteração de Valor para SSP MDP-IPs fatorados, com uma descrição detalhada da estrutura de dados utilizada no algoritmo, os PADDs.

\subsection{SSP MDP-IP Fatorado}

Um SSP MDP-IP fatorado é um SSP MDP-IP em que: i) os estados $\vec{x}$ são especificados como uma atribuição conjunta para um vetor $\vec{X}$ de $n$ variáveis de estado $\left(X_{1}, \ldots, X_{n}\right)$; ii) as redes credais dinâmicas (DCN, do inglês dynamic credal networks) (Cozman, 2000, 2005, Delgado et al., 2011) são utilizadas para representar a função de transição. $x_{i}$ é usado para representar o valor atribuído para uma variável de estado $X_{i}$. Neste trabalho é assumido que as variáveis são binárias para simplificar as notações, entretanto, estas definições podem ser facilmente estendidas para variáveis multivaloradas. A função custo também é dada em termos de um subconjunto de $\left\{X_{1}, \cdots, X_{n}\right\}$.

Nesta representação um estado abstrato pode ser considerado como uma atribuição parcial $\vec{w} \in\{0,1\}^{m}$, onde $m<n$. Por exemplo, para $n=3$, o estado abstrato $\vec{w}=\left\{X_{1}=1, X_{2}=0\right\}$ corresponde ao conjunto de estados $\left\{\left\{X_{1}=1, X_{2}=0, X_{3}=0\right\},\left\{X_{1}=1, X_{2}=0, X_{3}=1\right\}\right\}$.

Definição 5.1.1. (Conjunto credal de transição fatorado (Delgado et al., 2011))

Um conjunto credal contendo distribuições condicionais sobre os valores de uma variável $X_{i}$ dados os valores de pa $a_{a}\left(X_{i}\right)$ é referido como um conjunto credal de transição fatorado com respeito a ação $a \in A$ e é denotado por $K_{a}\left(x_{i} \mid p a_{a}\left(X_{i}\right), a\right)$.

Neste trabalho é assumido que as probabilidades imprecisas de um SSP MDP-IP fatorado são dadas em termos dos parâmetros de probabilidade sujeitas ao conjunto de restrições $\varphi$. Assim o conjunto credal de transição fatorado $K_{a}\left(x_{i} \mid p a_{a}\left(X_{i}\right), a\right)$, para $i=\{1, \cdots, n\}$ e $\forall a \in A$ satisfaz $\varphi$. Assim como na versão enumerativa, é assumido que todos os conjuntos credais de transição fatorados são fechados, convexos e não dependem do estágio $t$. 


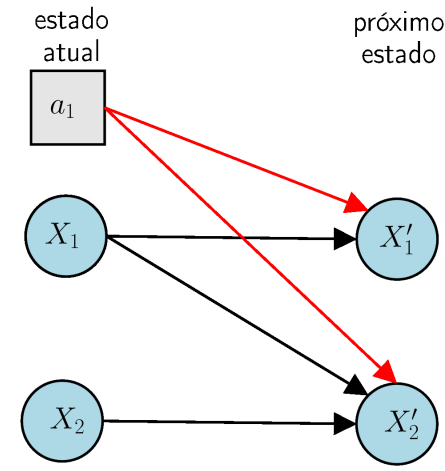

a)

CPT da ação $a_{1}$ para a variável $X_{1}^{\prime} \quad$ CPT da ação $a_{1}$ para a variável $X_{2}^{\prime}$

\begin{tabular}{c|l|l}
$X_{1}$ & $X_{1}^{\prime}=0$ & $X_{1}^{\prime}=1$ \\
\hline 0 & $p_{11}$ & $1-p_{11}$ \\
1 & $p_{12}$ & $1-p_{12}$
\end{tabular}

\begin{tabular}{cc|c|c}
$X_{1}$ & $X_{2}$ & $X_{2}^{\prime}=0$ & $X_{2}^{\prime}=1$ \\
\hline 0 & 0 & $p_{21}$ & $1-p_{21}$ \\
0 & 1 & $p_{22}$ & $1-p_{22}$ \\
1 & 0 & $p_{23}$ & $1-p_{23}$ \\
1 & 1 & $p_{24}$ & $1-p_{24}$
\end{tabular}

Conjunto de restrições $\varphi_{1}$ :

$0.3 \leq p_{11}$

$p_{12} \leq 0.8$

Conjunto de restrições $\varphi_{2}$

$0.5 \leq p_{21} \leq 0.7$

$0.3 \leq p_{22} \leq 0.5$

$0.25 \leq p_{23} \leq 0.35$

$0.1 \leq p_{24} \leq 0.2$

b)

c)

Figura 5.1: Rede Credal Dinâmica para a ação $a_{1} \in A$. a) Diagrama de dependência. b) CPT para a variável de estado $X_{1}^{\prime}$ e o conjunto de restrições $\varphi_{1}$. c) CPT para a variável de estado $X_{2}^{\prime}$ e o conjunto de restrições varphi $i_{2}$.

\section{Definição 5.1.2. (Rede Credal Dinâmica - DCN (Delgado et al., 2011))}

Uma Rede Credal Dinâmica é uma generalização de uma Rede Bayesiana Dinâmica (DBN, do inglês Dynamic Bayesian Network)) (Dean e Kanazawa, 1990) em que, dada uma ação a $\in A$, cada variável $X_{i}$ é associada com o conjunto credal de transição fatorado $K_{a}\left(x_{i} \mid p a_{a}\left(X_{i}\right), a\right)$ para cada valor de pa $a_{a}\left(X_{i}\right)$. Um DCN representa uma conjunção de conjuntos credais (Cozman, 2000, 2005) sobre todas as suas variáveis, consistindo em todas as distribuições que satisfazem:

$$
P\left(\vec{x}^{\prime} \mid \vec{x}, a\right)=\prod_{i=1}^{n} P\left(x_{i}^{\prime} \mid p a_{a}\left(X_{i}^{\prime}\right), a\right) .
$$

Para cada variável $X_{i}^{\prime}$ em uma DCN existe uma tabela de probabilidade condicional (CPT, do inglês conditional probability table) com probabilidades imprecisas, em que cada linha corresponde a uma possivel combinaçâo de valores de $p a_{a}\left(X_{i}^{\prime}\right)$, que definem um estado abstrato. As entradas de uma CPT são especificadas pelos parâmetros $p_{i j}$ ( $i$ para a variável $X_{i}^{\prime}, j$ para o j-ésimo parâmetro na CPT para $\left.X_{i}^{\prime}\right)$.

\section{Definição 5.1.3. (Stochastic Shortest Path com probabilidades imprecisas fatorado} (SSP MDP-IP fatorado))

Um MDP SSP-IP fatorado é definido por $\left\langle\vec{X}, A, C, \mathcal{K}, G, \overrightarrow{x_{0}}\right\rangle$, em que A é definido como qualquer SSP MDP-IP (Definição 4.1.3), $\vec{X}$ é um vetor de $n$ variáveis de estado $\left(X_{1}, \cdots, X_{n}\right), C$ é a função 
de custo fatorada, $\mathcal{K}$ é o conjunto de conjuntos credais de transição fatorados (Definição 5.1.1), $G$ é um conjunto de estados meta e $\overrightarrow{x_{0}}$ é o estado inicial.

A partir das Equações 4.1 e 5.1, a função valor ótima fatorada para SSP MDP-IPs é dada por:

$$
V^{*}(\vec{x})=\min _{a \in A}\left\{C(\vec{x}, a)+\max _{P \in K} \sum_{s^{\prime} \in S} \prod_{i=1}^{n} P\left(x_{i}^{\prime} \mid p a_{a}\left(X_{i}^{\prime}\right)\right) V^{*}\left(\vec{x}^{\prime}\right)\right\}, \forall \vec{x} \in\{0,1\}^{n} .
$$

Para resolver a Equação 5.2 primeiro é necessário calcular o produto (a probabilidade de transição conjunta) ou usar a eliminação de variáveis como o SPUDD-IP a ser explicado na Seção 5.2 .2 .

\subsubsection{Independência Par Estado abstrato-Ação e Número de Conjuntos Credais}

Para SSP MDP-IPs enumerativos foi assumido a independência do par estado-ação (Definição 4.1.2). No caso dos SSP MDP-IPs fatorados, ao invés de assumir independência sobre estados completos, a independência será assumida sobre estados abstratos, i.e., conjunto de estados que são definidos pela atribuição parcial de $p a_{a}\left(X_{i}^{\prime}\right)$.

Definição 5.1.4. (Independência do par estado abstrato-ação) As distribuições de probabilidade $P\left(X_{i}^{\prime} \mid p a_{a}\left(X_{i}^{\prime}\right), a\right)$ são independentes de um par estado abstrato-ação para outro.

Um exemplo de DCN é mostrado na Figura 5.1a. Os CPTs para as variáveis $X_{1}^{\prime}$ e $X_{2}^{\prime}$ são mostrados nas Figuras 5.1b e 5.1c. O conjunto de restrições $\varphi$ nestes $p_{i j}$ que são mostrados abaixo da CPT. O CPT da Figura 5.1b é definido por dois estados abstratos $\overrightarrow{w_{1}}=\left\{X_{1}=0\right\}$ e $\overrightarrow{w_{2}}=\left\{X_{1}=1\right\}$. Note que os parâmetros para as diferentes linhas dos CPTs (estados abstratos) são diferentes e as restrições também são independentes para diferentes estados abstratos.

O número de conjuntos credais $|\mathcal{K}|$ depende do número $n$ de variáveis de estado, no número de ações $|A|$ e da independência de variáveis de estado, i.e.:

$$
|\mathcal{K}| \leq|\mathcal{K}|_{\max }=n * 2^{\max _{a, X_{i}}\left|p a_{a}\left(X_{i}\right)\right|} *|A|,
$$

em que $\max _{a, X_{i}}\left|p a_{a}\left(X_{i}\right)\right|$ é o número máximo de pais em um problema para todas as variáveis de estado e ações. Por exemplo, para um domínio com 30 variáveis, $\max _{a, X_{i}}\left|p a_{a}\left(X_{i}\right)\right|=5$ e $|A|=10$, $|\mathcal{K}|_{\max }=300 \times 2^{5}$, enquanto a versão enumerativa tem $|\mathcal{K}|_{\max }=10 \times 2^{30}$.

\subsubsection{Gerando um SSP MDP-IP Enumerativo a partir de um SSP MDP-IP Fatorado}

Dado um SSP MDP-IP fatorado, é possível transforma-lo em seu correspondente enumerativo através do cálculo da probabilidade de transição como a conjunção de conjuntos credais de acordo com a Equação 5.1. A Figura 5.2 mostra o calculo da probabilidade conjunta para um SSP MDPIP fatorado dado na Figura 5.1. Por exemplo, para calcular a probabilidade de se estar no estado $\vec{x}^{\prime}=\left\{X_{1}^{\prime}=0, X_{2}^{\prime}=0\right\}$ dado que o estado atual do sistema é $\vec{x}=\left\{X_{1}^{\prime}=1, X_{2}^{\prime}=1\right\}$ e a ação $a_{1}$

foi tomada, é necessário multiplicar os parâmetros de probabilidade $p_{12}$ (Figura 5.1b) e $p_{24}$ (Figura 5.1c), i.e., $P\left(X_{1}^{\prime}=0, X_{2}^{\prime}=0 \mid X_{1}=1, X_{2}=1, a_{1}\right)=p_{12} p_{24}$, o que corresponde a $P\left(s_{0}^{\prime} \mid s_{3}, a_{1}\right)$ na matriz da Figura 5.2. Note que mesmo considerando os conjuntos de restrições lineares $\varphi_{1}$ e 


\begin{tabular}{|c|c|c|c|c|}
\hline$a_{1}$ & $s_{0}^{\prime}$ & $s_{1}^{\prime}$ & $s_{2}^{\prime}$ & $s_{3}^{\prime}$ \\
\hline$s_{0}$ & $p_{11} p_{21}$ & $p_{11}\left(1-p_{21}\right)$ & $\left(1-p_{11}\right) p_{21}$ & $\left(1-p_{11}\right)\left(1-p_{21}\right)$ \\
\hline$s_{1}$ & $p_{11} p_{22}$ & $p_{11}\left(1-p_{22}\right)$ & $\left(1-p_{11}\right) p_{22}$ & $\left(1-p_{11}\right)\left(1-p_{22}\right)$ \\
\hline$s_{2}$ & $p_{12} p_{23}$ & $p_{12}\left(1-p_{23}\right)$ & $\left(1-p_{12}\right) p_{23}$ & $\left(1-p_{12}\right)\left(1-p_{23}\right)$ \\
\hline$s_{3}$ & $p_{12} p_{24}$ & $p_{12}\left(1-p_{24}\right)$ & $\left(1-p_{12}\right) p_{24}$ & $\left(1-p_{12}\right)\left(1-p_{24}\right)$ \\
\hline & \multicolumn{2}{|c|}{$\begin{array}{l}\text { Conjunto de restrições } \varphi_{1} \text { : } \\
0.3 \leq p_{11} \\
p_{12} \leq 0.8\end{array}$} & \multicolumn{2}{|c|}{$\begin{array}{l}\text { Conjunto de restrições } \varphi_{2} \text { : } \\
0.5 \leq p_{21} \leq 0.7 \\
0.3 \leq p_{22} \leq 0.5 \\
0.25 \leq p_{23} \leq 0.35 \\
0.1 \leq p_{24} \leq 0.2\end{array}$} \\
\hline
\end{tabular}

Figura 5.2: Matriz de transição parametrizada completa $P\left(\cdot \mid s_{i}, a_{1}\right)$ calculada do DCN dado na Figura 5.1, onde $s_{0}=\left\{X_{1}=0, X_{2}=0\right\}, s_{1}=\left\{X_{1}=0, X_{2}=1\right\}, s_{2}=\left\{X_{1}=1, X_{2}=0\right\}$ e $s_{3}=\left\{X_{1}=1, X_{2}=1\right\}$.

$\varphi_{2}$ (Figura 5.2), agora existe um problema de otimização não-linear devido a multiplicação de parâmetros.

Assim, enquanto para um SSP MDP-IP enumerativo definido na Seção 4.1 as probabilidades de transição podem ser lineares (um único parâmetro de probabilidade $p_{i}$ para cada tripla $P\left(s^{\prime} \mid s, a\right)$ ), um SSP MDP-IP enumerativo gerado de um SSP MDP-IP fatorado é mais complexo, visto que agora existem parâmetros de probabilidades de transição conjuntas multilineares.

Note também que se as restrições são intervalos simples, como os das Figuras 5.1b e 5.1c , as mesmas podem definir um BMDP fatorado (Seção 4.3.1), porém quando são calculadas as probabilidades de transição conjuntas, não há mais um modelo baseado em intervalos e o algoritmo Iteração de Valor por Intervalos (Givan et al., 2000) para BMDPs não pode ser mais aplicado.

Como na prática os problemas são comumente descritos de maneira fatorada, pode-se assumir que qualquer SSP MDP-IP gerado de uma descrição fatorada terá que lidar com problemas de otimização não-lineares, mesmo se o conjunto de restrições sobre os parâmetros de probabilidades são intervalos simples.

\subsection{Diagramas de Decisão e SSP MDP-IPs Fatorados}

Uma extensão das ADDs, chamada de ADDs parametrizadas (PADDs, do inglês Parametrized $A D D s$ ), foi proposta por Delgado et al. (2011) para resolver de forma eficiente os MDP-IPs em um algoritmo chamado SPUDD-IP, que é mais rápido em duas ordens de magnitude em relação a Iteração de Valor utilizada para resolver os correspondentes MDP-IPs enumerativos com horizonte infinito.

\subsubsection{PADDs - ADDs Parametrizados}

Os PADDs (Delgado et al., 2011) são extensões dos ADDs utilizados para representar de forma compacta as funções $\{0,1\}^{n} \rightarrow \mathbb{E}$ sobre um conjunto de variáveis booleanas $Y$, onde $\mathbb{E}$ é o espaço de expressões sobre um conjunto de parâmetros $Z$, em que $Z_{i} \in Z(1 \leq i \leq k)$ pertence ao intervalo $[0,1]$ e com um conjunto de restrições sobre estes parâmetros. Por exemplo, as CPTs das Figuras 5.1b e 5.1c, podem ser representadas como PADDs (Figura 5.3) contendo nas folhas polinômios que envolvem os parâmetros de probabilidade $p_{i j}$ e o conjunto de restrições $\varphi$. 


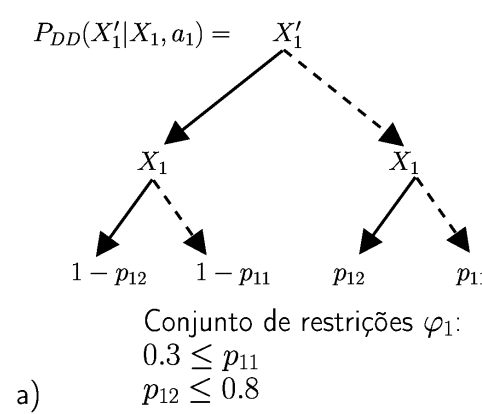

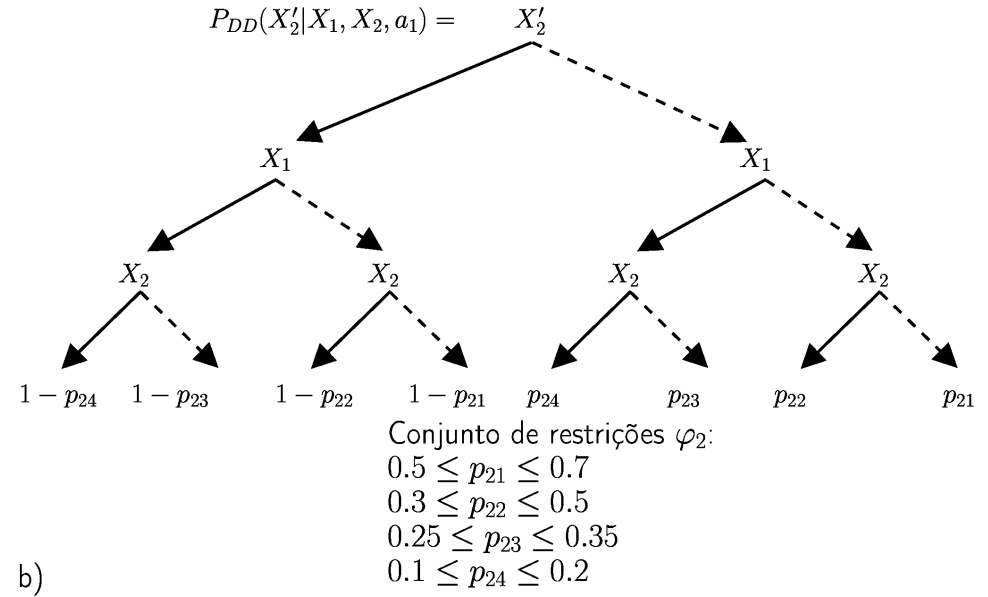

Figura 5.3: Representação usando PADDs das tabelas de probabilidade condicional da Figura $5.1 b$ e 5.1 c, denotadas por $P_{D D}\left(X_{1}^{\prime} \mid X_{1}, a_{1}\right)$ e $P_{D D}\left(X_{2}^{\prime} \mid X_{1}, X_{2}, a_{1}\right)$, respectivamente, e o conjunto de restrições $\varphi=$ $\varphi_{1} \cup \varphi_{2}$ sobre os parâmetros $p_{i j}$.

As expressões $\mathbb{E}$ podem ser restritas a polinômios, de forma que um PADD pode ser definido pela seguinte gramática BNF(Delgado et al., 2011):

$$
\begin{array}{ll}
F & ::=\operatorname{Poly} \mid \operatorname{se}\left(F_{\text {var }}=1\right) \text { então } F_{h} \text { senão } F_{l} \\
\text { Poly } & ::=\text { const } \mid Z_{i} * \text { Poly }+ \text { Poly }
\end{array}
$$

onde $Z_{i} \in Z$ está sujeita ao conjunto de restrições dado.

Note que um nó $F$ de um PADD pode ser um nó terminal com uma expressão do tipo Poly ou um nó de decisão com a variável de teste $F_{\text {var }}$ e duas ramificações do tipo não terminal $F$ (chamados de $F_{h}$ e $F_{l}$, respectivamente), onde $F_{h}$ é escolhido quando $F_{v a r}=1$ e $F_{l}$ é escolhido quando $F_{v a r}=0$.

As operações de ADDs podem ser facilmente estendidas e executas de forma eficiente em PADDs. As operações binárias: $\oplus$ (soma), $\ominus$ (subtração), e $\otimes$ (produto); as operações unárias: restrição, denotada por $\left.F\right|_{Y_{i}=y_{i}}$, pode ser calculada substituindo todos os nós de decisão da variável $Y_{i}$ com o ramo verdadeiro ou falso (veja um exemplo na Figura 5.4) e sum out (também chamada de marginalização) que elimina uma variável $Y_{i}$ do PADD através do cálculo $\sum_{y_{i} \in\{0,1\}} F=\left.F\right|_{Y_{i}=0}+\left.F\right|_{Y_{i}=1}$; e as operações n-árias: $\bigotimes_{i=1}^{n}$ (produto de uma sequência) e $\sum_{y_{1}, \cdots, y_{n} / y_{i} \in\{0,1\}}$ (múltipla marginalização que calcula $n$ sequências de operações sum out, i.e. $\left.\sum_{y_{1}, \cdots, y_{n}} F=\sum_{y_{n}}\left(\cdots\left(\sum_{y_{2}}\left(\sum_{y_{1}} F\right)\right) \cdots\right)\right)$ são fechadas para PADDs (i.e., estas operações em PADDs produzem novos PADDs com folhas que também são expressas como Poly). ${ }^{1}$

A seguir, cinco operações para PADDs serão definidas (Definições 5.2.1, 5.2.2, 5.2.3, 5.2.4 e 5.2.5) para serem utilizadas nos algoritmos fatorados que serão propostos no Capítulo 6. As descrições mais detalhadas das operações mais básicas em PADDs podem ser vistas em (Delgado et al., 2011).

Definição 5.2.1. (Avaliação de um PADD - EvalPADD $(F, \vec{y})$ )

Dado o conjunto de variáveis de teste $Y$ com a atribuição de variáveis $\vec{y} \in\{0,1\}^{n}$, o polinômio retornado pela operação de avaliação de um PADD, nomeada EvalPADD $(F, \vec{y})$, pode ser calculada

\footnotetext{
${ }^{1}$ Por questões de simplificação ao invés de $\sum_{y_{1}, \cdots, y_{n} / y_{i} \in\{0,1\}}$ será utilizado $\sum_{y_{1}, \cdots, y_{n}}$ e ao invés de $\sum_{y_{i} \in\{0,1\}}$ será utilizado $\sum_{y_{i}}$
} 


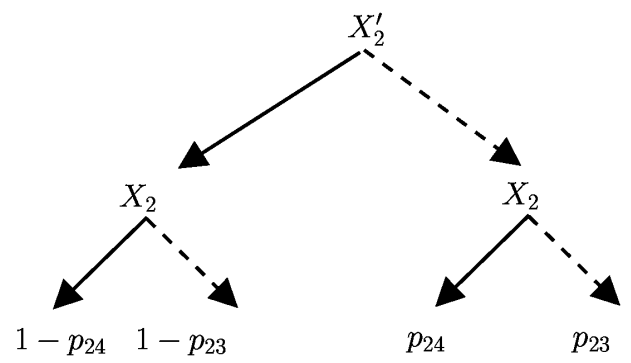

Figura 5.4: Um exemplo da operação de restrição sobre o PADD da Figura $5.3 b$ para a variável $X_{1}=1$.

a)

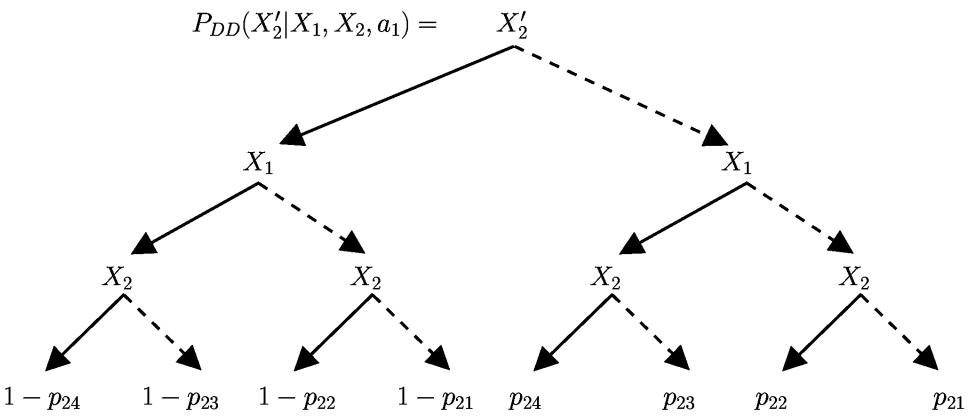

)

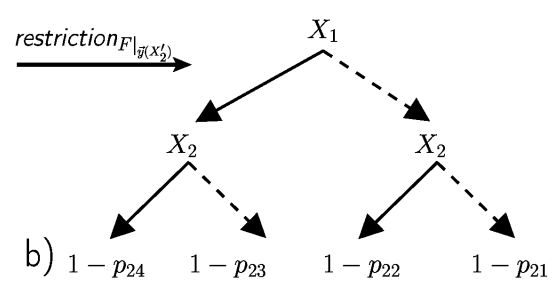

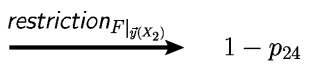

d)

Figura 5.5: Avaliação do PADD para a função $P_{D D}\left(X_{2}^{\prime} \mid X_{1}, X_{2}, a_{1}\right)$ da Figura $5.3 b$ com $\vec{y}=\left\{X_{1}^{\prime}=\right.$ $\left.1, X_{2}^{\prime}=1, X_{1}=1, X_{2}=1\right\}$. a) $O P A D D$ antes da avaliação. b) $O P A D D$ resultante após aplicar a restrição $\left(\left.F\right|_{X_{2}^{\prime}=\vec{y}\left(X_{2}^{\prime}\right)}\right)$. c) O PADD resultante após aplicar a restrição $\left(\left.F\right|_{X_{1}=\vec{y}\left(X_{1}\right)}\right)$. d) O PADD resultante após aplicar a restrição $\left(\left.F\right|_{X_{2}=\vec{y}\left(X_{2}\right)}\right)$.

recursivamente por:

$$
\operatorname{EvalPADD}(F, \vec{y})= \begin{cases}\text { Poly } & \text { se } F=\text { Poly } \\ \operatorname{EvalPADD}\left(F_{h}, \vec{y}\right) & \text { se } F \neq \text { Poly } \wedge \vec{y}\left(F_{\text {var }}\right)=1 \\ \operatorname{EvalPADD}\left(F_{l}, \vec{y}\right) & \text { se } F \neq \text { Poly } \wedge \vec{y}\left(F_{\text {var }}\right)=0 .\end{cases}
$$

em que $\vec{y}\left(F_{\text {var }}\right)$ é o valor de $F_{\text {var }}$ na atribuição de variáveis $\vec{y}$.

$\operatorname{EvalP} A D D(F, \vec{y})$ pode ser calculada aplicando a restrição para $\left.\left(\left(\left.\left(\left.F\right|_{Y_{1}=\vec{y}\left(Y_{1}\right)}\right)\right|_{Y_{2}=\vec{y}\left(Y_{2}\right)}\right) \ldots\right)\right|_{Y_{n}=\vec{y}\left(Y_{n}\right)}$ resultando em um nó terminal Poly.

A Figura 5.5 dá um exemplo de $\operatorname{EvalPADD}(F, \vec{y})$, em que $F$ é a função de transição probabilística $P_{D D}\left(X_{2}^{\prime} \mid X_{1}, X_{2}, a_{1}\right), n=4$ e $\vec{y}$ é uma atribuição para as variáveis de estado $X_{1}^{\prime}, X_{2}^{\prime}, X_{1}$ e $X_{2}$. O resultado é o polinômio $1-p_{24}$.

Definição 5.2.2. (Avaliação parcial de um PADD - pEvalPADD $(F, \vec{w})$ )

Dado uma atribuição parcial de variáveis $\vec{w} \in\{0,1\}^{m}, m<n$ com $W \subset Y$, a avaliação parcial de um PADD, nomeada pEvalPADD $(F, \vec{w})$, retorna um PADD (ao invés de um polinômio) e é calculada por $\left.\left(\left(\left.\left(\left.F\right|_{W_{1}=\vec{w}\left(W_{1}\right)}\right)\right|_{W_{2}=\vec{w}\left(W_{2}\right)}\right) \ldots\right)\right|_{W_{m}=\vec{w}\left(W_{m}\right)}$. 


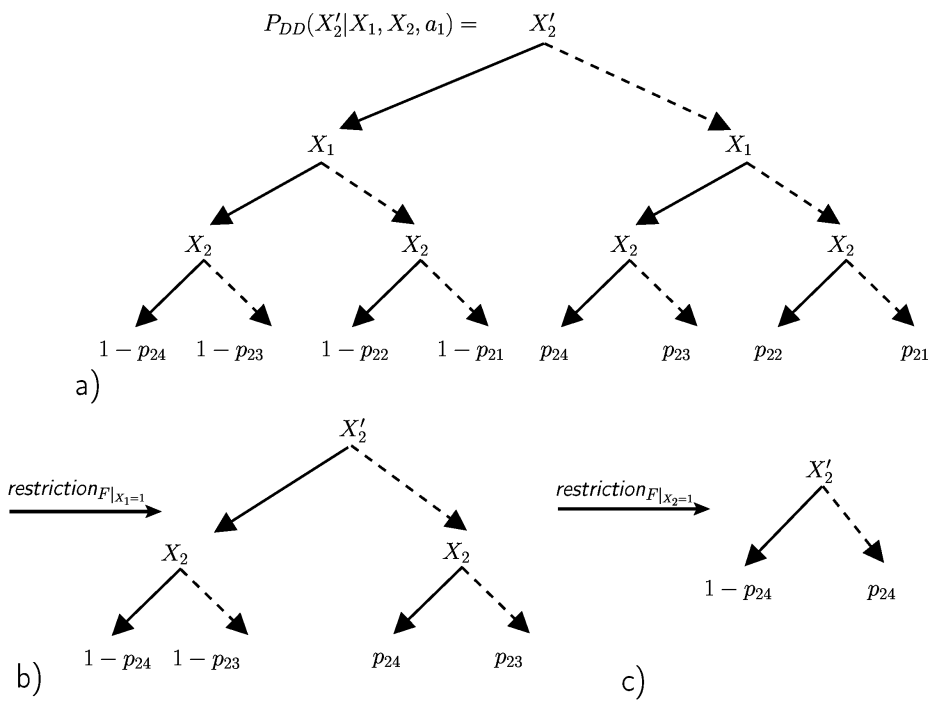

Figura 5.6: Avaliação parcial do PADD $P_{D D}\left(X_{2}^{\prime} \mid X_{1}, X_{2}, a_{1}\right)$ da Figura $5.3 b$ com $\vec{w}=\left\{X_{1}=1, X_{2}=1\right\}$. a) $O P A D D$ antes da avaliação. b) $O P A D D$ resultante após aplicar a restrição $\left(\left.F\right|_{X_{1}=\vec{w}\left(X_{1}\right)}\right)$. c) $O P A D D$ resultante após aplicar a restrição $\left(\left.F\right|_{X_{2}=\vec{w}\left(X_{2}\right)}\right)$.

A Figura 5.6 exemplifica esta operação, em que $F$ é a função de transição probabilística $P_{D D}\left(X_{2}^{\prime} \mid X_{1}, X_{2}, a_{1}\right)$ e $\vec{w}$ é uma atribuição para as variáveis de estado $X_{1}$ e $X_{2}$, que resulta em um PADD com a variável $X_{2}^{\prime}$.

Definição 5.2.3. (Valor da folha de um PADD - ValPoly $(P o l y, \vec{z})$ )

Dado um polinômio Poly (i.e. uma folha de um PADD) contendo um conjunto de parâmetros $Z$ e uma atribuição $\vec{z} \in[0,1]^{k}$ sobre $Z$, o valor de Poly de acordo com $\vec{z}$, nomeado $\operatorname{ValPoly}($ Poly, $\vec{z})$ pode ser definido recursivamente como:

$$
\operatorname{ValPoly}(\text { Poly }, \vec{z})=\left\{\begin{array}{ll}
\text { const } & \text { se Poly }=\text { const } \\
\vec{z}\left(Z_{i}\right) * \operatorname{ValPoly}\left(\text { Poly }_{1}, \vec{z}\right)+\operatorname{ValPoly}\left(\text { Poly }_{2}, \vec{z}\right) & \text { se Poly } \neq \text { const }
\end{array} .\right.
$$

em que $\vec{z}\left(Z_{i}\right)$ é o valor de $Z_{i}$ na atribuição de parâmetros $\vec{z}$.

Por exemplo, dado $k=4$, um polinômio Poly $=p_{12} p_{22}$ e $\vec{z}=\left\{p_{11}=0.5, p_{12}=0.3, p_{21}=\right.$ $\left.0.65, p_{22}=0.4\right\}, \operatorname{ValPoly}($ Poly, $\vec{z})$ será 0.12 .

Definição 5.2.4. (Todos os valores das folhas de um PADD - ValPADDLeaves $(F, \vec{z})$ )

Dado um PADD F contendo um conjunto de $k$ parâmetros $Z$ e uma atribuição $\vec{z} \in[0,1]^{k}$ sobre $Z$, a função ValPADDLeaves $(F, \vec{z})$ retorna um ADD calculando o valor de cada folha de $F$ de acordo com $\vec{z}$, aplicando ValPoly(Poly, $\vec{z})$ (Equação 5.6).

A função $\operatorname{ValPADDLeaves}(F, \vec{z})$ pode ser recursivamente calculada construindo um ADD canônico, começando dos nós folha terminais (substituídos pelo valor retornado por $\operatorname{ValPoly}(\operatorname{Poly}, \vec{z}))$ ) até o nó raiz.

Um exemplo desta função é dado na Figura 5.7 em que $k=4, \vec{z}=\left\{p_{11}=0.5, p_{12}=0.3, p_{21}=\right.$ $\left.0.65, p_{22}=0.4\right\}$ e $F$ é a função de transição probabilística $P_{D D}\left(X_{1}^{\prime} \mid X_{1}, a_{1}\right)$, que resulta no PADD da Figura 5.7b.

Definição 5.2.5. (MaxParameterOut - MaxParameterOut $(F, \varphi)$ (Delgado et al., 2011)) Dado um PADD F com um conjunto de parâmetros $Z$ tal que $|Z|=k$ e o conjunto de restriçôes $\varphi$ 


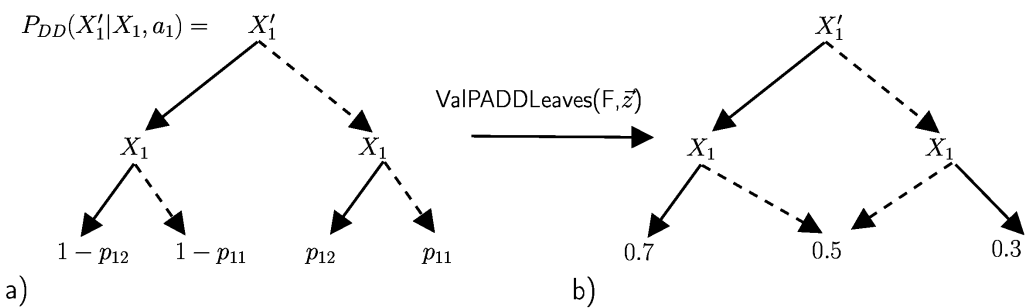

Figura 5.7: Substituição de parâmetros do PADD para a função $P_{D D}\left(X_{1}^{\prime} \mid X_{1}, a_{1}\right)$ da Figura $5.3 a$ com $k=4$ parâmetros, $\vec{z}=\left\{p_{11}=0.5, p_{12}=0.3, p_{21}=0.65, p_{22}=0.4\right\}$. a) PADD antes da avaliação. b) $P A D D$ resultante depois de aplicar ValPADDLeaves $(F, \vec{z})$.

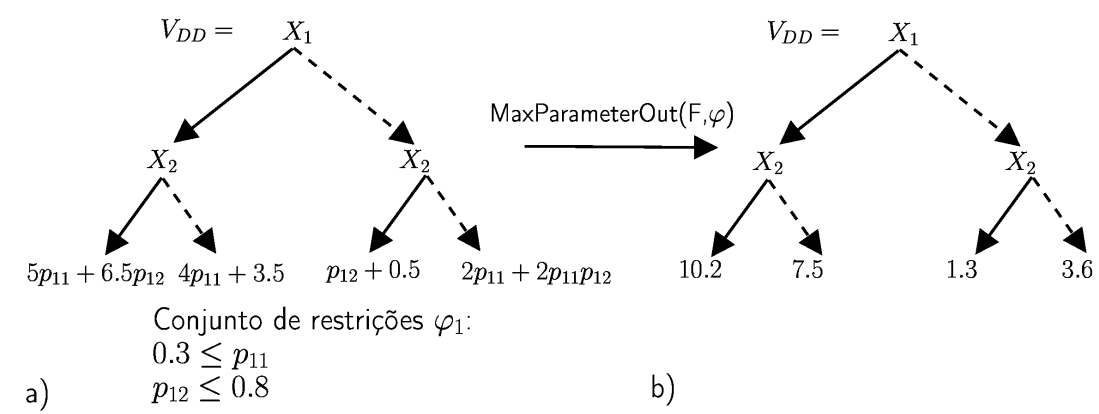

Figura 5.8: Exemplo da função MaxParameterOut. a) PADD $V_{D D}$ antes de aplicar a função e o conjunto de restrições $\varphi_{1}$. b) ADD resultante após aplicar MaxParameterOut $(F, \varphi)$.

sobre $Z$, MaxParameterOut $(F, \varphi)$ retorna um ADD, onde cada folha polinomial Poly é substituída pelo valor retornado pela chamada de um solver não-linear com $\max _{Z_{1}, \cdots, Z_{k}}$ Poly s.t. $\varphi$.

A função MaxParameterOut $(F, \varphi)$ pode ser calculada recursivamente de forma similar a função $\operatorname{ValPADDLeaves}(F, \vec{z})$.

Um exemplo da função MaxParameterOut é dado na Figura 5.8 onde $F$ é a função valor $V_{D D}$ e $\varphi_{1}$ é o conjunto de restrições, que resultam no ADD da Figura 5.8b.

\subsubsection{O Algoritmo SPUDD-IP}

Satia e Lave Jr.(1970) foram os primeiros a propor um algoritmo exato de Iteração de Valor para MDP-IPs enumerativos de horizonte infinito. Uma solução eficiente foi proposta por Delgado et al. (2011), para resolver MDP-IPs utilizando PADDs em todas as etapas do algoritmo Iteração de Valor, chamado SPUDD-IP. Esse algoritmo adaptado para resolver SSP MDP-IP define as Equações (4.2) e (4.3) usando uma representação fatorada da função de transição probabilística (Equação (5.1)) e da função de custo. Assim um Bellman update fatorado para todos os estados é dado por ${ }^{2}$ :

$$
V_{D D}^{t+1}(\vec{X})=\min _{a \in A}\left\{Q_{D D}^{t+1}(\vec{X}, a)\right\}
$$

\footnotetext{
${ }^{2}$ Neste trabalho, a notação $D D$ será utilizada em funções representadas por ADDs e PADDs.
} 


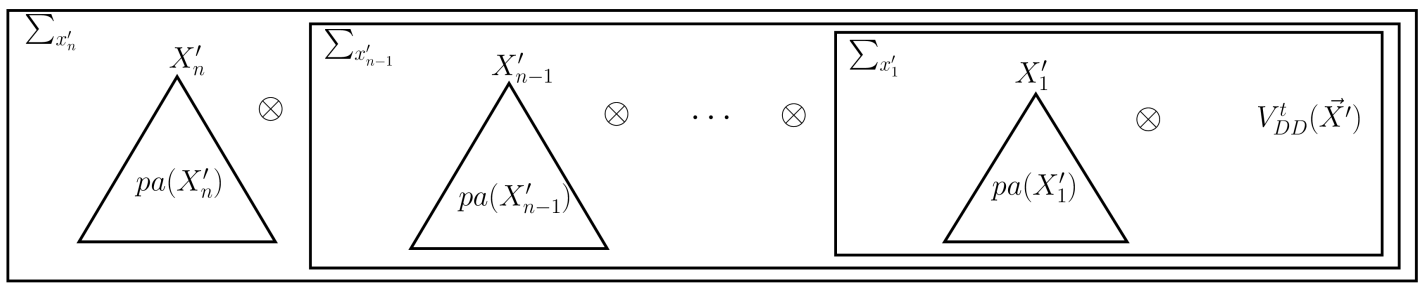

Figura 5.9: Eliminação de variáveis no SPUDD-IP. O triângulo representa $P_{D D}\left(X_{i}^{\prime} \mid p a_{a}\left(X_{i}^{\prime}\right), a\right)$. Dentro da caixa com o triângulo para $X_{i}^{\prime}$, a operação sum out é realizada sobre os valores de $X_{i}$.

em que,

$$
\begin{aligned}
& Q_{D D}^{t+1}(\vec{X}, a)=C_{D D}(\vec{X}, a) \oplus \\
& \max _{\vec{p} \in K_{a}} \sum_{x_{1}^{\prime}, x_{n}^{\prime}} \bigotimes_{i=1}^{n}\left(P_{D D}\left(X_{i}^{\prime} \mid p a_{a}\left(X_{i}^{\prime}\right), a\right)\right) \otimes V_{D D}^{t}\left(\vec{X}^{\prime}\right) .
\end{aligned}
$$

Todas as operações nas Expressões (5.7) e (5.8) podem ser calculadas utilizando operações de diagramas de decisão em que a função custo e a função valor para todos os estados são ADDs (denotados por $C_{D D}$ e $V_{D D}^{t}$ ); e as funções de transição (CPTs) são representadas como PADDs, denotados por $P_{D D}\left(X_{i}^{\prime} \mid p a_{a}\left(X_{i}^{\prime}\right), a\right)$, uma para cada par da variável $X_{i}^{\prime}$ e ação $a$. Note que a maximização $\max _{\vec{p} \in K_{a}}$ na Equação 5.8 é realizada aplicando a operação MaxParameterOut em um PADD, i.e. chamando um solver não linear cada folha do PADD, resultando em um ADD (esta operação corresponde a escolher o pior modelo para um par estado abstrato-ação). Portanto $Q_{D D}^{t+1}$ também é um ADD. Isto é importante porque a operação $\min _{a \in A}{ }^{3}$ na Equação 5.7 só pode ser realizada entre ADDs. Usando PADDs, o SPUDD-IP realiza operações sobre um conjunto de estados (i.e., estados abstratos) que tem o mesmo valor e precisam somente chamar um solver não-linear para cada folha do PADD, não necessariamente para todos os estados. Esta é a chave para melhorar a eficiência em ordens de magnitude. Para realizar cálculos eficientes, o SPUDD-IP explora o algoritmo de eliminação de variável (Zhang e Poole, 1994) utilizando a operação sum out sobre os PADDs (expressão no retângulo na Equação (5.8)). A idéia é que se uma variável $X_{i}^{\prime}$ não depende de todas as outras variáveis $X_{j}^{\prime}$, é possível empurrar a operação sum out $\sum_{X_{i}^{\prime}}$ dentro de $\bigotimes_{i=1}^{n}$. A Figura 5.9 mostra como a eliminação de variáveis pode ser realizada para todas as variáveis $X_{i}^{\prime}$ se a ordem topológica for escolhida baseando-se nas DCNs da ação a. Nesta figura, o triângulo representa o PADD $P_{D D}\left(X_{i}^{\prime} \mid p a_{a}\left(X_{i}^{\prime}\right), a\right)$ e as caixas aninhadas indicam a sequência de eliminação de variáveis (iniciando da caixa mais interna para a mais externa). Note que desde que $V_{D D}^{t}\left(\vec{X}^{\prime}\right)$ tem somente variáveis "linha" e a sequência de sum outs é feita também para todas as variáveis "linhas", o resultado da expressão no retângulo da Equação 5.8 é um PADD com variáveis não "linha". O critério de parada para o SPUDD-IP é quando o resíduo de Bellman $B E_{I P}$ fica menor que um limite $\epsilon$ (Equação 4.5).

Duas extensões da Iteração de Valor foram propostas: (i)APRICODD-IP, um extensão direta da versão aproximada do SPUDD (APRICODD (St-Aubin et al., 2000)), para MDP-IPs e (ii) Objective-IP (Delgado et al., 2011), que tenta converter as folhas polinomiais em constantes de forma a atacar a fonte principal de complexidade de tempo para a resolução de um MDP-IP,

\footnotetext{
${ }^{3} \min _{a \in A}$ pode ser calculada aplicando uma sequência de operações binárias $\min (\cdot, \cdot)$.
} 
i.e., o número de chamadas para o solver não linear durante a iteração de valor em um MDPIP (Delgado et al., 2011).

Apesar destas soluções serem o estado-da-arte para MDP-IPs de horizonte infinito, elas podem não ser a melhor solução para SSP MDP-IPs. Assim, baseado na comparação entre os resultados das soluções para MDP-IPs com horizonte infinitos fatorados e MDP-IPs com horizonte infinitos enumerativos (Delgado et al., 2011), serão propostas as soluções assíncronas enumerativas (Seção 6.1) e fatoradas (Seção 6.3) para SSP MDP-IPs. 


\section{Capítulo 6}

\section{Programação Dinâmica Assíncrona para SSP MDP-IPs}

Neste capítulo são propostas as primeiras soluções assíncronas para SSP MDP-IP: o algoritmo RTDP-IP, que envolve problemas de otimização, baseado no algoritmo RTDP (apresentado na Seção 3.2.1) para SSP MDPs (Barto et al., 1995), sua extensão, o LRTDP-IP, baseado no algoritmo LRTDP para SSP MDPs (Bonet e Geffner, 2003) e duas versões fatoradas destes algoritmos chamados de factRTDP-IP e factLRTDP-IP. Além disso, adaptações dos algoritmos SSiPP e LSSiPP são propostas, chamadas de SSiPP-IP e LSSiPP-IP.

\subsection{O Algoritmo RTDP-IP}

O algoritmo RTDP-IP se baseia nas idéias do algoritmo RTDP para MDPs enumerativos (apresentado na Seção 3.2.1), modificando o algoritmo nos seguintes pontos: (i) o Bellman update e (ii) a escolha do próximo estado.

Assim como no algoritmo RTDP (Algoritmo 4), o RTDP-IP (Algoritmo 12) inicializa a função valor com uma função valor inicial (Algoritmo 12, linha 3). O algoritmo também inicializa as tabelas hash utilizadas para a amostragem do próximo estado (Algoritmo 12, linhas 4 a 9) a serem detalhados posteriormente nesta seção. Os trials são realizados pelo algoritmo (Algoritmo 12, linhas 13 a 19) e visam explorar o espaço de estados a partir do estado inicial $s_{0}$, até alcançar um estado meta (Algoritmo 12, linha 13).

A cada visita a um estado $s$, o algoritmo executa um Bellman update no estado com as Equações 4.2 e 4.3 (Algoritmo 12, linha 15) que são calculadas pelo método RTDP-IP-UPDATE (Algoritmo 13). Note que, em relação a Iteração de Valor, este Bellman update considera apenas o estado atual que está sendo visitado no trial. Nesta atualização o estado $s^{\prime}$ é considerado como um sucessor do estado $s$, de acordo com a ação gulosa $a$, se o parâmetro probabilístico $p_{i}=P\left(s^{\prime} \mid s, a\right)$ pode assumir pelo menos um valor maior que 0 . Os estados sucessores são obtidos através da chamada do método SuccessorStates-IP (Algoritmo 13, linha 4).

Após o Bellman uptade, o algoritmo realiza uma "simulação" da execução de uma ação, escolhendo a ação gulosa $a$ e realizando um sorteio do próximo estado $s^{\prime}$ considerando as probabilidades imprecisas, isto é, dada uma ação gulosa, primeiro os valores para cada $p_{i}$ são escolhidos, sujeitos ao conjunto de restrições $\varphi$, para depois fazer a escolha real (Algoritmo 12, linha 19). O algoritmo 

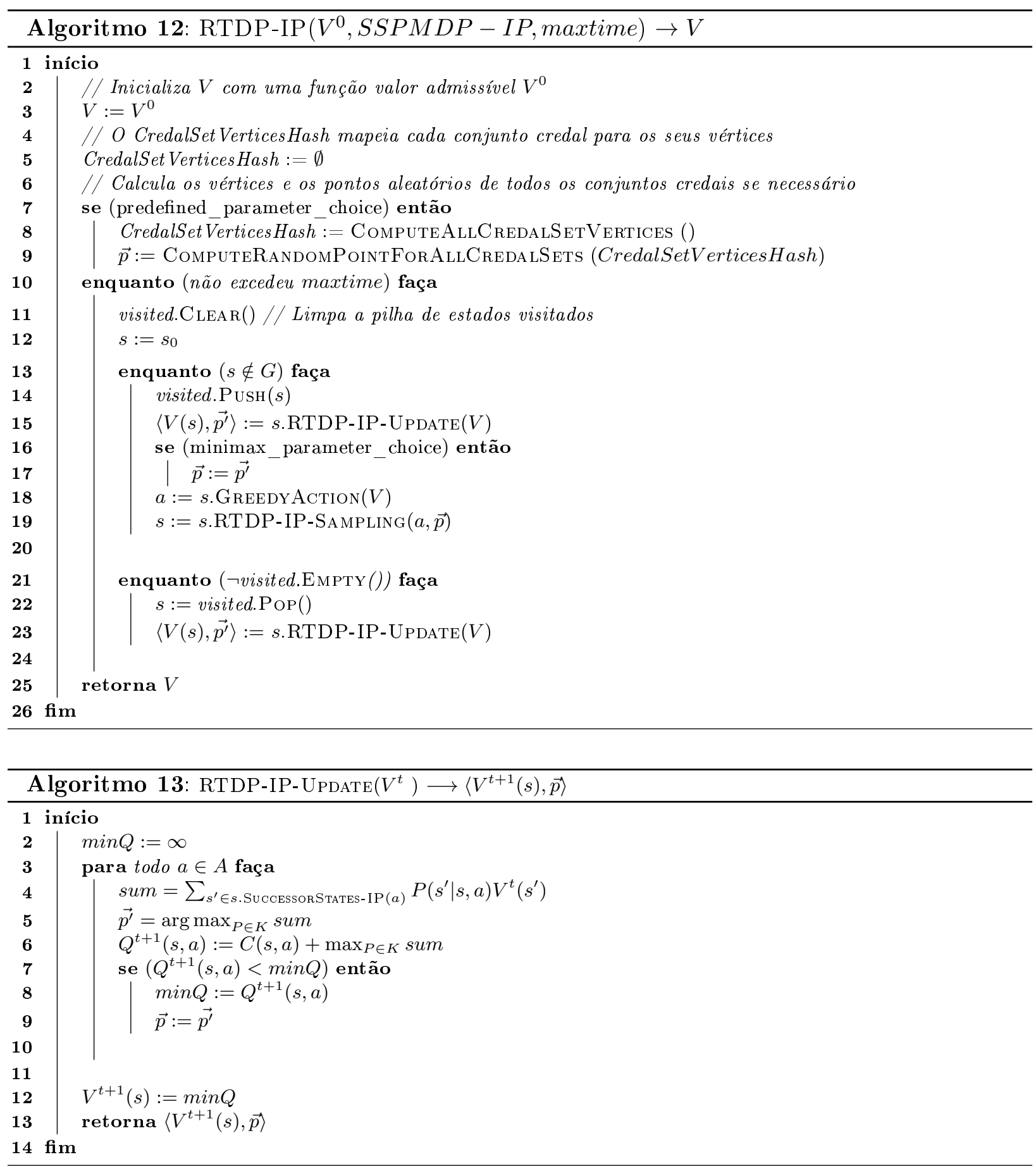

RTDP-IP-SAMPLing (Algoritmo 14) será explicado na próxima subseção.

Por fim, assim como no RTDP, o RTDP-IP realiza uma atualização da função valor para os estados visitados (Linhas 21 a 23) a fim de refinar a função valor para estes estados.

\subsubsection{Amostragem de Estados no RTDP-IP}

Para escolher um valor para cada parâmetro probabilístico $p_{i}$, sujeito ao conjunto de restrições $\varphi$, três métodos são considerados: (1) escolher o mesmo valor computado pelo Bellman update (método minimax_parameter_choice); (2) calcular um valor aleatório válido a cada visita de um estado durante o trial (método rand_parameter_choice); (3) calcular um valor válido pré determinado 


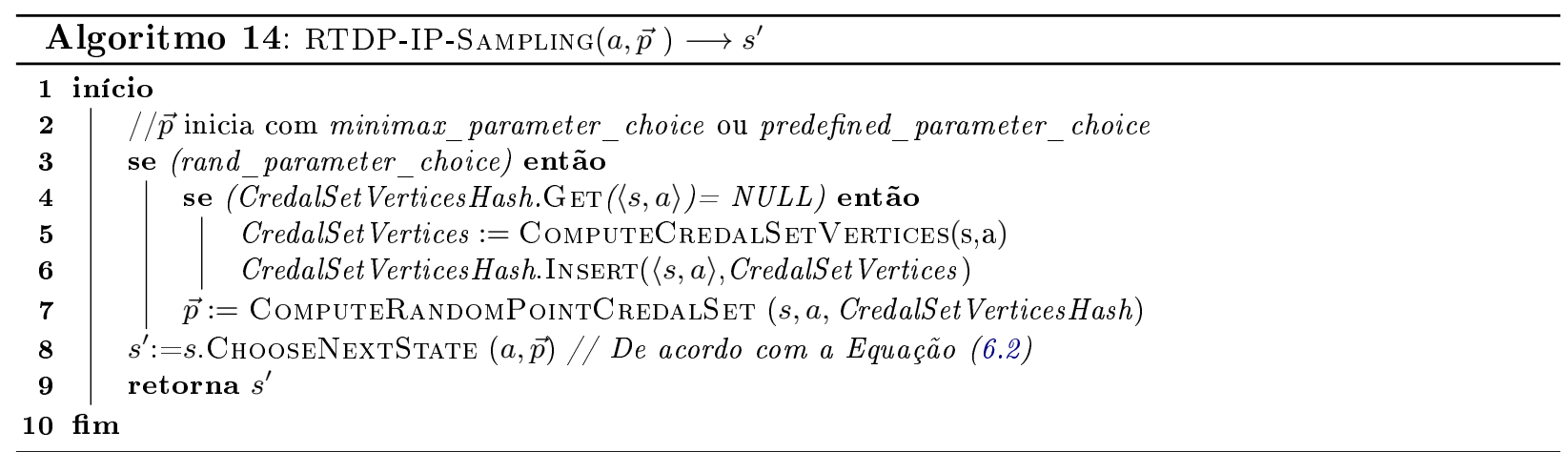

apenas uma vez no início do algoritmo (método predefined_parameter_choice).

O método minimax_parameter_choice utiliza o mesmo $\vec{p} \in K$ calculado na Linha 15 (Algoritmo 12) pelo método RTDP-IP-UPDATE. Neste método, $\vec{p}$ pode ser utilizado para ambos os propósitos, tanto para atualizar o valor de um estado quanto para amostrar o próximo estado.

Os métodos predefined_parameter_choice e rand_parameter_choice calculam distribuições de probabilidade válidas sob o conjunto de conjuntos credais. Assumindo que os conjuntos credais dos SSP MDP-IPs são fechados e convexos, é possível obter o vértices $\overrightarrow{u_{0}}, \cdots, \overrightarrow{u_{l}}$ de cada conjunto credal $K(\cdot \mid s, a)$ (cada vértice é uma distribuição sobre $S$ ) utilizando o software LRS (Avis, 2000) que enumera os vértices de um politopo definido pelo conjunto de restrições $\varphi$.

Por exemplo, considere um problema em que para o estado $s_{0}$ e ação $a_{2}$ existem apenas três estados sucessores possíveis $s_{3}, s_{4}$ e $s_{5} \mathrm{com}$ as probabilidades de transição dadas pelos parâmetros $p_{1}, p_{2}$ e $p_{3}$, i.e., $P\left(s_{3} \mid s_{0}, a_{2}\right)=p_{1}, P\left(s_{4} \mid s_{0}, a_{2}\right)=p_{2}$ e $P\left(s_{5} \mid s_{0}, a_{2}\right)=p_{3}$, definidas com as seguintes restrições: $\varphi_{1}=\left\{p_{1} \leq 2 / 3, p_{3} \leq 2 / 3,2 p_{1} \geq p_{2}, p_{1}+p_{2}+p_{3}=1\right\}$. A região onde todas as medidas de probabilidade que satisfazem $\varphi_{1}$ é mostrada na Figura 6.2 onde os vértices do politopo (região em cinza) são: $\overrightarrow{u_{0}}=\{1 / 3,0,2 / 3\}, \overrightarrow{u_{1}}=\{0,1 / 3,2 / 3\}, \overrightarrow{u_{2}}=\{1 / 3,2 / 3,0\}, \overrightarrow{u_{3}}=\{2 / 3,1 / 3,0\}$ e $\overrightarrow{u_{4}}=\{2 / 3,0,1 / 3\}$. Uma distribuição de probabilidade válida (i.e., um ponto dentro da região em cinza) pode ser $\vec{p}=\{0.5,0.2,0.3\}$, que é uma combinação linear de $\overrightarrow{u_{j}}$.

Assim, os vértices do politopo definidos para cada conjunto credal $K(\cdot \mid s, a)$ pode ser utilizado para gerar um ponto aleatório $\vec{p} \in K(\cdot \mid s, a)$ como uma combinação linear de $\overrightarrow{u_{j}}$ (Devroye, 1986), que é:

$$
\sum_{j=0}^{l} w_{j} * \overrightarrow{u_{j}},
$$

em que $w_{j}$ é gerado por uma amostragem aleatória sobre $[0,1]$ e depois normalizado para garantir que $\sum_{j=0}^{l} w_{j}=1$.

O método predefined_parameter_choice primeiro calcula todos os vértices dos conjuntos credais (Algoritmo 12, Linha 8) e depois usa a função ComputeRandomPointForAllCRedalSets para calcular os pontos aleatórios $\vec{p} \in K$ (Equação (6.1)) para todos os conjuntos credais (Algoritmo 12, Linha 9), i.e. para os conjuntos credais $K(\cdot \mid s, a), \forall s \in S$ e $\forall a \in A$. Esse método calcula esses pontos aleatórios antes de iniciar os trials e usa o mesmo $\vec{p}$ para cada par estado-ação.

O método rand_parameter_choice calcula um ponto aleatório (Equação (6.1)) para o conjunto credal $K(\cdot \mid s, a)$, onde $s$ é o estado atual no trial e a é a ação gulosa (Algoritmo 14, Linhas 3-7). Assim, enquanto o método predefined_parameter_choice calcula $\vec{p}$ para cada par estado-ação antes 


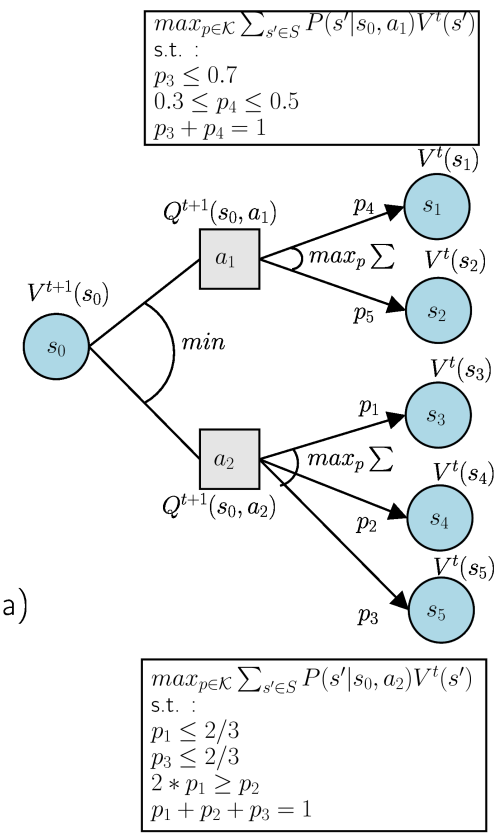

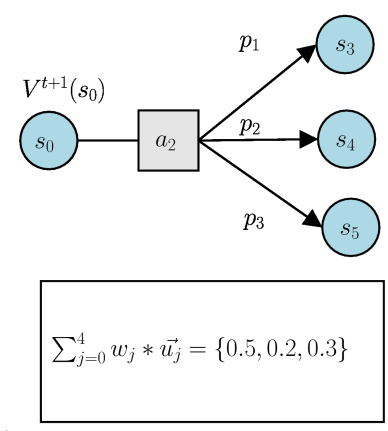

b)

Figura 6.1: Bellman update do RTDP-IP e a amostragem do próximo estado utilizando o método rand_parameter_choice. a) O RTDP-IP chama duas vezes o solver linear, para as ações $a_{1}$ e $a_{2}$ e depois escolhe a ação gulosa $a_{2}$; b) $O$ método rand_parameter_choice que calcula a Equação (6.1) para escolher um ponto aleatório do politopo mostrado na Figura 6.2, i.e., $p_{1}=0.5 p_{2}=0.2$ e $p_{3}=0.3$; c) A amostragem do próximo estado para o RTDP-IP.

de iniciar os trials, o método rand_parameter_choice calcula $\vec{p}$ somente para o estado visitado e a ação gulosa correspondente. Note que os vértices $\overrightarrow{u_{0}}, \cdots, \overrightarrow{u_{l}}$ para o conjunto credal $K(\cdot \mid s, a)$ são armazenados em uma tabela hash chamada CredalSetVerticesHash (Algoritmo 14, Linha 6). Estes vértices podem ser reusados se $s$ é revisitado e $a$ continua sendo a ação gulosa para calcular o novo $\vec{p}$.

Após escolher os valores para $\vec{p}$, com um dos três métodos apresentados, pode-se fazer a amostragem do próximo estado da seguinte maneira:

$$
\text { s.ChoosenextState }(a, \vec{p})=s^{\prime} \sim P(\cdot \mid s, a),
$$

em que $P(\cdot \mid s, a)$ é completamente conhecido, i.e., todos os parâmetros de probabilidade, $\vec{p}$, são conhecidos.

A Figura 6.1 ilustra um exemplo de um Bellman update para um SSP MDP-IP e a amostragem do próximo estado. Para o Bellman update, a Figura 6.1a mostra duas chamadas para o solver linear que irão escolher a medida de probabilidade que maximiza o custo futuro do agente seguindo a ação $a_{1}$ ou $a_{2}$, sendo a ação $a_{2}$ a que minimiza os resultados. A Figura $6.1 \mathrm{~b}$ ilustra o cálculo de $\vec{p}$ usando o método rand_parameter_choice com a ação gulosa $a_{2}$ que resulta em $p_{1}=0.5, p_{2}=0.2$ e $p_{3}=0.3$. Finalmente, com os valores dos parâmetros $p_{1}, p_{2}$ e $p_{3}$ escolhidos, o RTDP-IP amostra o próximo estado (Figure 6.1c).

\subsubsection{Convergência do RTDP-IP}

Para a prova de convergência do RTDP-IP, este trabalho seguirá a mesma linha da prova do trabalho de Buffet e Aberdeen (2005) para o RTDP robusto, visto que a imprecisão pode ser referida como um conjunto de possíveis modelos em um politopo definido por um conjunto credal. 


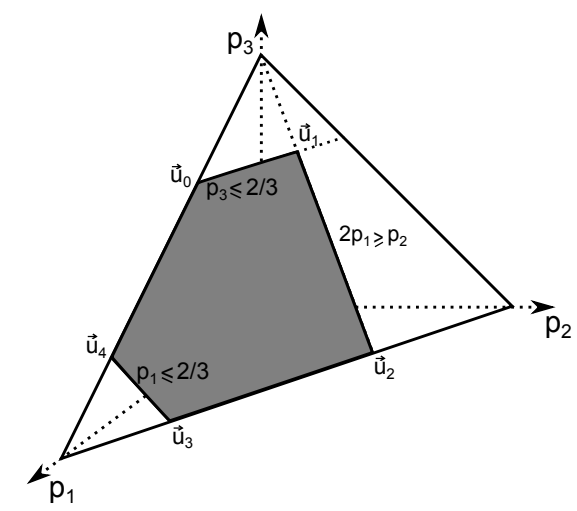

Figura 6.2: Um exemplo de conjunto credal de transição representado pela área em cinza.

Entretanto, dois pontos precisam ser esclarecidos nesta prova:

1. A convergência da programação dinâmica assíncrona para SSP MDP-IPs, para ser completa, deve se basear no fato que o operador $\mathrm{T}$ da Equação 4.2 é uma contração (Teorema 6.1.1).

2. A preservação da admissibilidade da função valor, que não é provada por Buffet (Proposição $6.1 .2)$.

Teorema 6.1.1. A programação dinâmica assíncrona para SSP MDP-IP converge para $V^{*}$ se o valor de cada estado é atualizado infinitas vezes.

Prova: A prova segue diretamente do fato que o operador de atualização para jogos SSP sequenciais e em particular o operador T dos SSP MDP-IPs (Equação 4.2) é uma contração a respeito da norma do supremo (sup-norm, Lema 4.1 em Patek e Bertsekas (1999)). Isto é uma condição suficiente para a convergência da programação dinâmica assíncrona para SSP MDP-IPs (Bertsekas, 1982).

Em segundo lugar, é mostrado que a admissibilidade da função valor de um SSP MDP-IP é preservada durante os trials do RTDP-IP.

Proposição 6.1.2. Se o valor inicial $V^{0}$ é admissivel, a admissibilidade da função valor é preservada durante a execução do RTDP-IP, i.e., se $V^{0}(s) \leq V^{*}(s), \forall s \in S$ então $V^{t}(s) \leq V^{*}(s), \forall s \in S$ e $t \in \mathbb{N}^{*}$.

Prova: Seja $s_{t}$ o estado atual, $a_{t}$ a ação gulosa e $V^{t}$ a função valor no instante de tempo $t$, gerada pela execução do RTDP-IP começando de um estado inicial arbitrário. É verdade que $V^{t}(s) \leq V^{*}(s), \forall s \in S$ pois $V^{t}(s)=V^{t-1}(s), \forall s \neq s_{t}$ e por indução se $V^{t-1}\left(s^{\prime}\right) \leq V^{*}\left(s^{\prime}\right), \forall s^{\prime} \in S$, então para todo $t$ :

$$
\begin{aligned}
V^{t}\left(s_{t}\right) & =\min _{a \in A}\left\{C\left(s_{t}, a\right)+\max _{P \in K}\left\{\sum_{s^{\prime} \in S} P\left(s^{\prime} \mid s_{t}, a\right) V^{t-1}\left(s^{\prime}\right)\right\}\right\} \\
& \leq \min _{a \in A}\left\{C\left(s_{t}, a\right)+\max _{P \in K}\left\{\sum_{s^{\prime} \in S} P\left(s^{\prime} \mid s_{t}, a\right) V^{*}\left(s^{\prime}\right)\right\}\right\}=V^{*}\left(s_{t}\right) .
\end{aligned}
$$


Note que a única diferença em relação a prova de Barto é a adição de $\max _{P \in K}$ na Equação (6.3), que também implica na mesma inequalidade. Assim, a prova do RTDP de Barto para a preservação da admissibilidade foi facilmente estendida para o RTDP-IP.

Para completar a prova de convergência, é necessário garantir que todos os estados relevantes serão visitados infinitas vezes. Para isto é necessário garantir que o método que escolhe $P \in K$ para amostrar o próximo estado nunca elimina nenhum estado que possa eventualmente ser visitado. Nesta proposta, visto que rand_parameter_choice escolhe um $P \in K$ diferente para cada amostragem do próximo estado, ele eventualmente ira visitar todos os estados alcançáveis. Entretanto, para os outros métodos (minimax_parameter_choice e predefined_parameter_choice) é necessário evitar a escolha de uma probabilidade $P\left(s^{\prime} \mid s, a\right) \in K(\cdot \mid s, a)$ igual a 0 . Por exemplo, o método predefined_parameter_choice pode escolher $P\left(s^{\prime} \mid s, a\right) \in K(\cdot \mid s, a)$ igual a 0 para algum $s^{\prime} \in S$ dado $(s, a)$ e utilizá-lo depois, assim nunca seria possível visitar $s^{\prime}$. No pior caso, o mesmo pode acontecer se o método minimax_parameter_choice escolhe $P\left(s^{\prime} \mid s, a\right) \in K(\cdot \mid s, a)$ igual a 0 para algum $s^{\prime} \in S$ a cada vez que visita o estado $s$ com a ação $a$. Para garantir a convergência utilizando estes dois métodos é necessário escolher outro $P\left(s^{\prime} \mid s, a\right) \in K(\cdot \mid s, a)$ diferente de 0 utilizando a Equação 6.1 .

Assim, o teorema de convergência do RTDP-IP é definido como:

Teorema 6.1.3. (Convergência do RTDP-IP com diferentes métodos de amostragem para o próximo estado) Em um SSP MDP-IP, repetidos trials do RTDP-IP eventualmente irão gerar valores ótimos sobre todos os estados relevantes se: (i) a função valor inicial é admissivel, i.e., $V^{0}(s) \leq V^{*}(s)$ e (ii) o método de escolha de $P\left(s^{\prime} \mid s, a\right) \in K(\cdot \mid s, a)$ para amostrar o próximo estado garante que $P\left(s^{\prime} \mid s, a\right) \in K(\cdot \mid s, a)$ não será igual a 0 , para algum $s^{\prime} \in S$ dado $(s, a)$.

\subsection{O Algoritmo LRTDP-IP}

Para melhorar a convergência, é possível modificar o Algoritmo 12 para o Labeled RTDP-IP, chamado LRTDP-IP (Algoritmo 15). Diferente do ChEckSolved (Bonet e Geffner, 2003) para SSP MDP, a função CHEckSolved-IP (chamada no Algoritmo 15, linha 27) calcula o grafo guloso considerando todos os estados que podem ser alcançados com a política gulosa com probabilidade maior que 0 .

\subsection{O Algoritmo factRTDP-IP}

Baseado nas soluções assíncronas fatoradas para MDPs (Holguin, 2013), o algoritmo factRTDPIP combina as idéias do SPUDD-IP (Delgado et al., 2011) (Seção 5.2.2) e do RTDP-IP (Seção 6.1).

factRTDP-IP Update Assim como o RTDP, o Bellman update é realizado somente para o estado que está sendo visitado no trial, conforme a seguinte Equação:

$$
V^{t+1}(\vec{x})=\min _{a \in A}\left\{Q^{t+1}(\vec{x}, a)\right\}
$$




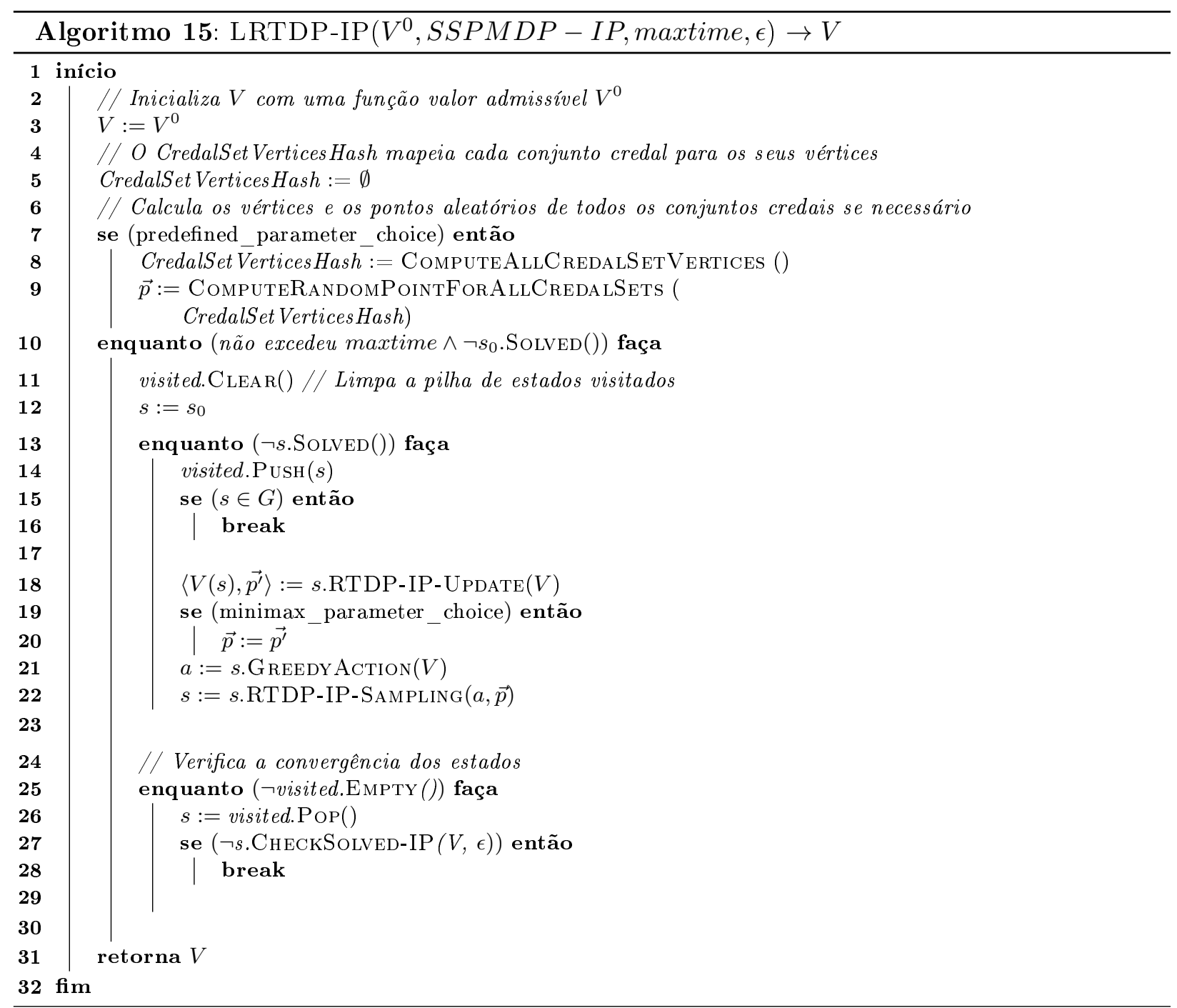

em que

$$
\begin{aligned}
& Q^{t+1}(\vec{x}, a)=\operatorname{EvalPADD}\left(C_{D D}(\vec{X}, a), \vec{x}\right) \oplus \\
& \max _{\vec{p} \in K_{a}} \sum_{x_{1}^{\prime}, \cdots x_{n}^{\prime}} \bigotimes_{i=1}^{n}\left(p E v a l P A D D\left(P_{D D}\left(X_{i}^{\prime} \mid p a_{a}\left(X_{i}^{\prime}\right), a\right), \vec{x}\right)\right) \otimes V_{D D}^{t}\left(\vec{X}^{\prime}\right)
\end{aligned}
$$

Note que, após calcular a Equação $(6.5) Q^{t+1}(\vec{x}, a)$ e $V^{t+1}(\vec{x})$ viram valores reais, de forma diferente da ADD resultante calculada pelo backup do SPUDD-IP (Equações 5.7 e 5.8), em que $Q_{D D}^{t+1}(\vec{X}, a)$ e $V_{D D}^{t+1}(\vec{X})$ são ADDs representando os valores correspondentes para o conjunto completo de estados. Isto ocorre devido ao algoritmo explorar o espaço de estados em trials e realizar Bellman updates somente para o estado atualmente visitado, fazendo com que seja obtido somente o valor constante $V^{t+1}$ para o estado $\vec{x}$.

Como no SPUDD-IP, o cálculo da Equação (6.5) pode ser realizada de forma eficiente eliminando uma variável de cada vez, desde que ela não dependa de outra variável. Por exemplo, se $X_{1}^{\prime}$ não é dependente de outra $X_{i}^{\prime}, \forall i \neq 1$ utilizando a ação $a$, é possível "empurrar" a soma sobre $X_{1}^{\prime}$ para dentro, expandindo a Equação (6.5) em: 


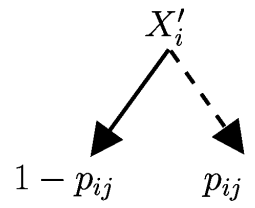

Figura 6.3: $P A D D$ resultante da operação pEvalP $A D D\left(P_{D D}\left(X_{i}^{\prime} \mid p a_{a}\left(X_{i}^{\prime}\right), a\right), \vec{x}\right)$.

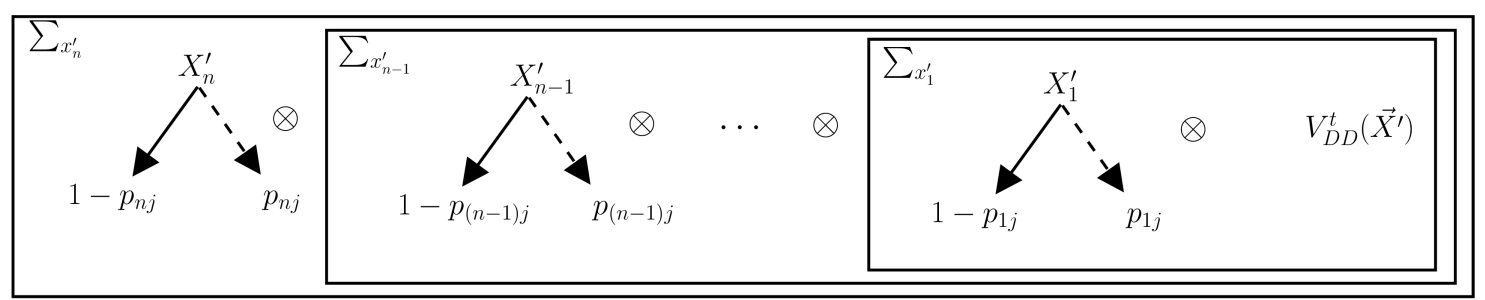

Figura 6.4: Eliminação de variáveis no factRTDP-IP.

$$
\begin{aligned}
Q^{t+1}(\vec{x}, a) & =\operatorname{EvalPADD}\left(C_{D D}(\vec{X}, a), \vec{x}\right) \oplus \\
& \max _{\vec{p} \in K_{a}} \sum_{x_{1}^{\prime}, \cdots, x_{n}^{\prime}} \bigotimes_{i=2}^{n} p \operatorname{EvalPADD}\left(P_{D D}\left(X_{i}^{\prime} \mid p a_{a}\left(X_{i}^{\prime}\right), a\right), \vec{x}\right) \otimes \\
& \sum_{\overrightarrow{x_{1}}{ }^{\prime}} p \operatorname{EvalP} A D D\left(P_{D D}\left(X_{1}^{\prime} \mid p a_{a}\left(X_{1}^{\prime}\right), a\right), \vec{x}\right) \otimes V_{D D}^{t}\left(\vec{X}^{\prime}\right),
\end{aligned}
$$

que pode ser repetida para todas as variáveis, obtendo:

$$
\begin{aligned}
Q^{t+1}(\vec{x}, a) & =p \operatorname{EvalPADD}\left(C_{D D}(\vec{X}, a), \vec{x}\right) \oplus \\
& \max _{\vec{p} \in K_{a}} \sum_{x_{n}} p \operatorname{EvalPADD}\left(P_{D D}\left(X_{n}^{\prime} \mid p a_{a}\left(X_{n}^{\prime}\right), a\right), \vec{x}\right) \otimes \\
& \ldots \\
& \sum_{\overrightarrow{x_{2}}} p \operatorname{EvalPADD}\left(P_{D D}\left(X_{2}^{\prime} \mid p a_{a}\left(X_{2}^{\prime}\right), a\right), \vec{x}\right) \otimes \\
& \sum_{\vec{x}^{\prime}} p E v a l P A D D\left(P_{D D}\left(X_{1}^{\prime} \mid p a_{a}\left(X_{1}^{\prime}\right), a\right), \vec{x}\right) \otimes V_{D D}^{t}\left(\vec{X}^{\prime}\right) .
\end{aligned}
$$

Como $X_{i}^{\prime}$ representa uma variável $X_{i}$ no próximo estado e $p a_{a}\left(X_{i}^{\prime}\right)$ os pais de $X_{i}^{\prime}$, $p E v a l P A D D\left(P_{D D}\left(X_{i}^{\prime} \mid p a_{a}\left(X_{i}^{\prime}\right)\right), \vec{x}\right)$, que aplica a operação de restrição para cada variável de estado, resulta em um PADD mostrado na Figura 6.3, com uma única variável $X_{i}^{\prime}$.

A Figura 6.4 mostra como a eliminação de variável ocorre (considerando a ordem topológica das variáveis $X_{i}^{\prime}$ ). Um exemplo desta operação pode ser visto na Figura 6.5 para o estado visitado $\vec{x}=\left\{X_{1}=1, X_{2}=1\right\}$ e a ação $a$. A Figura 6.5 a mostra o produto entre a primeira função valor $V_{D D}^{0}\left(\vec{X}^{\prime}\right)$ (obtida após colocar "linha" as variáveis de $V_{D D}^{0}(\vec{X})$ ) e as probabilidades de transição para $X_{1}^{\prime}$ dado o estado atual $\vec{x}$ (i.e., o PADD resultante após aplicar a operação pEvalPADD $\left.\left(P_{D D}\left(X_{1}^{\prime} \mid p a_{a}\left(X_{1}^{\prime}\right), a\right), \vec{x}\right)\right)$; Na Figura $6.5 \mathrm{~b}$, com o resultado do último produto, a variável $X_{1}^{\prime}$ é eliminada aplicando a operação de marginalização sobre $x_{1}^{\prime}$. Após esta operação, $X_{2}^{\prime}$ 
a)

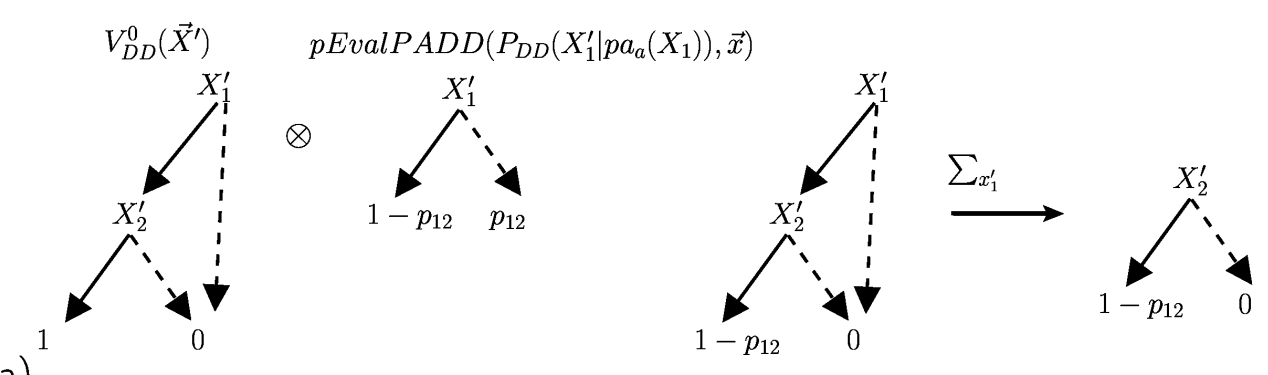

b)

$p \operatorname{EvalPADD}\left(P_{D D}\left(X_{2}^{\prime} \mid p a_{a}\left(X_{2}\right)\right), \vec{x}\right)$

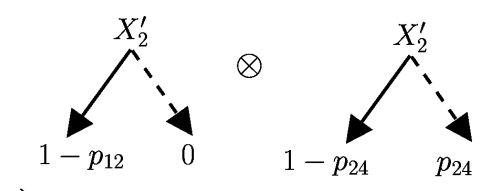

c)

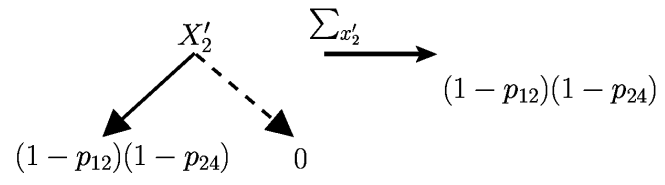

d)

Figura 6.5: Exemplo das operações de PADD para calcular a Equação (6.7) para o estado visitado $\vec{x}=$ $\left\{X_{1}=1, X_{2}=1\right\}$ e a ação $a$.

pode ser eliminada multiplicando o resultado anterior pelas probabilidades de transição de $X_{2}^{\prime}$ dado o estado atual $\vec{x}$ e a ação $a$ (Figura $6.5 \mathrm{c}$ ). Finalmente, na Figura 6.5d a marginalização sobre $x_{2}^{\prime}$. é aplicada. Note que a PADD resultante corresponde a um polinômio multilinear. Isso é importante pois para realizar otimizações multilineares existem implementações eficientes que podem ser utilizadas na prática.

Em seguida, para realizar a maximização sobre os parâmetros na Equação (6.5), a operação MaxParameterOut é aplicada. Note que maximizar os parâmetros em PADDs é equivalente a maximizar parâmetros nas folhas. Ela é realizada através da chamada de um solver não linear para uma folha no PADD, que resulta em um ADD com uma folha, i.e., que corresponde a um valor real. Para o exemplo mostrado na Figura 6.5, o solver é chamado para $\left(1-p_{12}\right)\left(1-p_{24}\right)$ (Figura 6.5d) e para o conjunto de restrições $\varphi$, retornando os valores $\left\{p_{11}=0, p_{12}=0.8, p_{21}=0.5, p_{22}=0.5, p_{23}=0.25, p_{24}=0.15\right\} \mathrm{e}$ o valor do polinômio. Esse valor é usado com $\operatorname{EvalP} A D D\left(C_{D D}(\vec{X}, a), \vec{x}\right)$ para calcular $Q^{t+1}(\vec{x}, a)$.

Finalmente, na Equação (6.4) a minimização sobre os valores de $Q^{t+1}$ para todas as ações é realizada, resultando na constante $V^{t+1}$, que o factRTDP-IP insere na função valor atual $V_{D D}^{t}$ para obter $V_{D D}^{t+1}$. Esta inserção é feita construindo um ADD de função indicador $\vec{x}_{D D}$ que tem o valor 1 para o estado $\vec{x}$ e 0 para os outros, i.e., dado a atribuição de variáveis $\vec{v}$ :

$$
\operatorname{Eval} A D D\left(\vec{x}_{D D}, \vec{v}\right)= \begin{cases}1 & \text { if } \vec{v}=\vec{x} \\ 0 & \text { caso contrário. }\end{cases}
$$

Uma maneira de construir a função indicadora $\vec{x}_{D D}$ é utilizar a operação de produto sequencial $\bigotimes_{i=1}^{n} X_{i D D}$, em que $X_{i D D}$ é o PADD de uma variável:

$$
\left(x_{i}\right)^{X_{i}}\left(1-x_{i}\right)
$$

que associa $x_{i}$ para a ramificação verdadeira e $\left(1-x_{i}\right)$ para a ramificação falsa.

Assim, o valor $V^{t+1}(\vec{x})$ pode ser "mascarado" na antiga função valor $V_{D D}^{t}(\vec{X})$ para se obter a função atualizada $V_{D D}^{t+1}(\vec{X})$ através do cálculo $V_{D D}^{t} \oplus\left(\left(V^{t+1}(\vec{x})-V^{t}(\vec{x})\right) \otimes \vec{x}_{D D}\right)$. Um exemplo é dado 


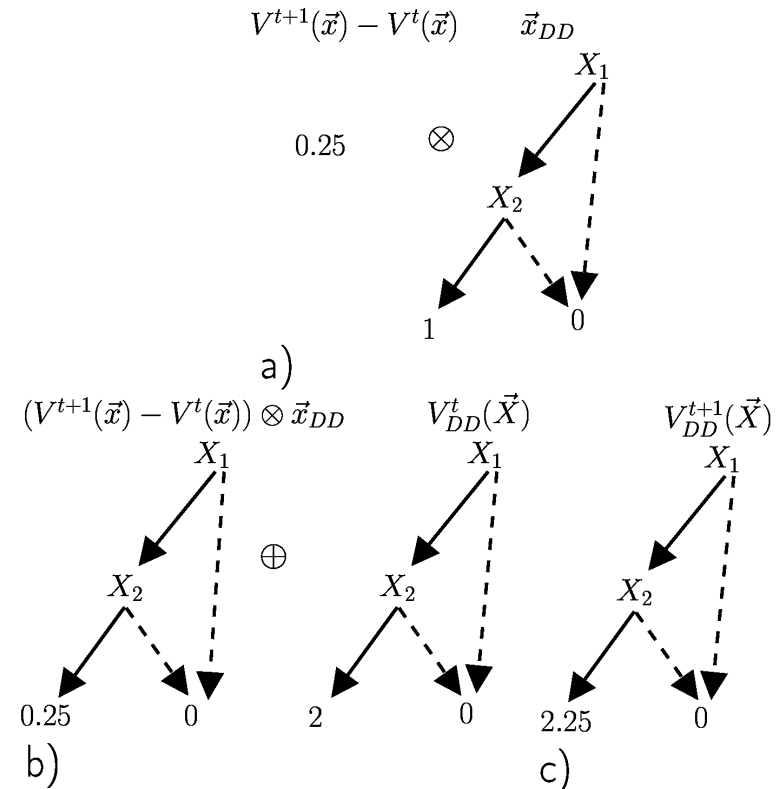

Figura 6.6: Inserção de $V^{t+1}(\vec{x})$ em $V_{D D}^{t}(\vec{X})$ : o novo valor 2.25 para o estado $\vec{x}=\left\{X_{1}=1, X_{2}=1\right\}$ calculado pela Equação (6.4), dado que o antigo valor era 2. a) Calcula o produto da diferença entre $V^{t+1}(\vec{x})$ e $V^{t}(\vec{x})$ e a função indicadora $\vec{x}_{D D}$ que tem o valor 1 para o estado $\vec{x}$ e 0 para os demais. b) Obtém o resultado e soma com a antiga função valor $V_{D D}^{t}(\vec{X})$. c) Por fim, $V_{D D}^{t+1}(\vec{X})$, o ADD resultante de calcular $V_{D D}^{t} \oplus\left(\left(V^{t+1}(\vec{x})-V^{t}(\vec{x})\right) \otimes \vec{x}_{D D}\right)$.

na Figura 6.6. Nesta notação é utilizado $D D$ para funções representadas por ADDs e por PADDs; $\vec{x}$ para representar um estado e $\vec{X}$ para representar um vetor com variáveis de estado binárias.

Amostragem de estados no factRTDP-IP Nesta operação, os métodos apresentados na Seção 6.1 são novamente considerados para se escolher o valor dos parâmetros de probabilidade, i.e., minimax_parameter_choice, predefined_parameter_choice e rand_parameter_choice. Note que para o segundo e o terceiro método, como feito na Seção 6.1.1, é necessário calcular os vértices dos conjuntos credais de transição fatorados. Após obter os valores para $\vec{p}$, o factRTDP-IP realiza a amostragem de cada variável $X_{i}^{\prime}$ do próximo estado baseados no CPT da ação $a$ escolhida, i.e.:

$$
\vec{x} \cdot \operatorname{NeXtStateVar}\left(X_{i}^{\prime}, a, \vec{p}\right)=x_{i}^{\prime} \sim P(\cdot \mid \vec{x}, a),
$$

em que a distribuição de probabilidades $P(\cdot \mid \vec{x}, a)$ é obtida a partir das folhas do ADD retornado por ValPADDLeaves $\left(p E v a l P A D D\left(P_{D D}\left(X_{i}^{\prime} \mid p a_{a}\left(X_{i}^{\prime}\right), a\right), \vec{x}\right), \vec{p}\right)$.

Note que este ADD tem uma única variável $X_{i}^{\prime}$ (i.e., o resultado da avaliação de $P_{D D}$ com as atribuições de variáveis $\vec{x}$ e os parâmetros de probabilidade $\vec{p}$ ). A Figura 6.7 mostra um exemplo do processo de escolha do próximo estado $\vec{x}^{\prime}$, dado que o estado atual é $\vec{x}=\left\{X_{1}=1, X_{2}=1\right\}$ e os valores de parâmetros escolhidos são $p_{11}=0, p_{12}=0.8, p_{21}=0.5, p_{22}=0.5, p_{23}=0.25$ e $p_{24}=0.15$. A Figura 6.7 mostra como amostrar as variáveis $X_{1}^{\prime}$ e $X_{2}^{\prime}$.

O algoritmo FACTRTDP-IP (Algoritmo 16) inicializa $V_{D D}$ como um ADD com uma função valor admissível e realiza uma série de trials que vão até atingir um estado meta. Para cada estado visitado, o algoritmo FACTRTDP-IP-UPDATE é chamado (Algoritmo 16, Linha 16) retornando a função valor atualizada e os valores dos parâmetros que podem ser reusados pelo método minimax_parameter_choice. Após escolher a ação gulosa (Algoritmo 16, Linha 19), a amostragem do 
a)
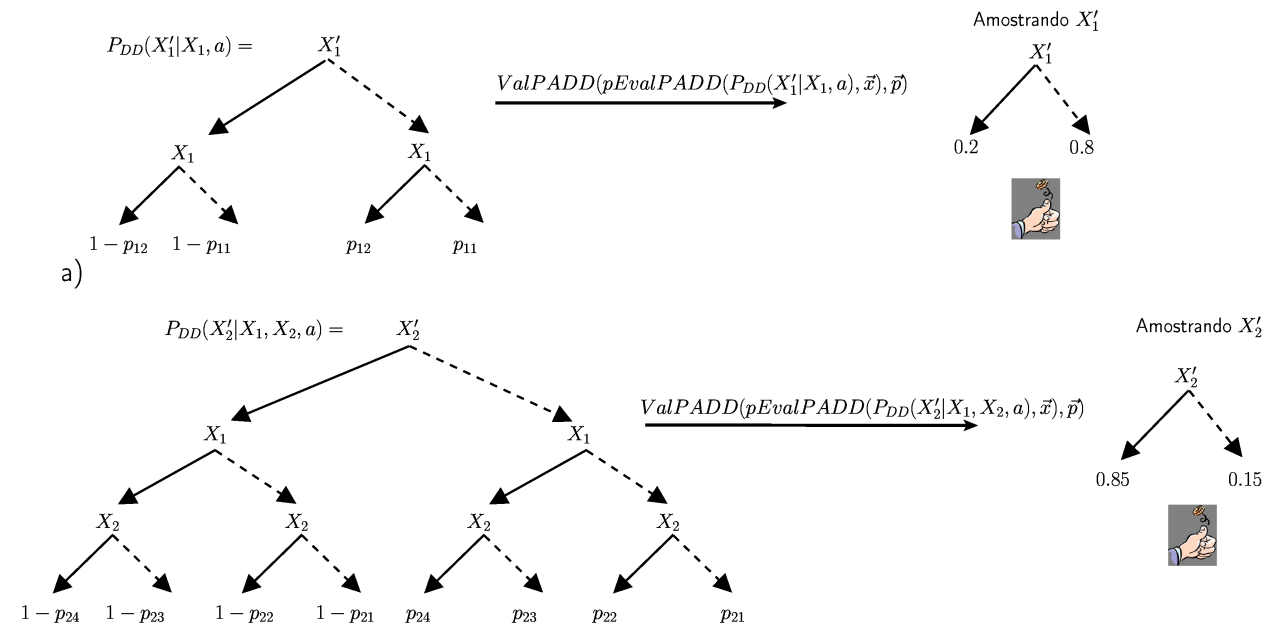

b)

Figura 6.7: Amostragem do factRTDP-IP para as variáveis de estado $X_{1}^{\prime}$ e $X_{2}^{\prime}$ dado o estado atual visitado $\vec{x}=\left\{X_{1}=1, X_{2}=1\right\}$ e os valores para os parâmetros $\vec{p}$. a) Aplicação de $\operatorname{ValPADD}\left(p\right.$ EvalPADD $\left.\left(P_{D D}\left(X_{1}^{\prime} \mid X_{1}, a_{1}\right), \vec{x}\right), \vec{p}\right)$ e amostragem do valor para $X_{1}^{\prime}$ com 0.2 para $X_{1}^{\prime}=1$ e 0.8 para $X_{1}^{\prime}=0$. b) ValPADD $\left(p\right.$ EvalPADD $\left.\left(P_{D D}\left(X_{2}^{\prime} \mid X_{1}, X_{2}, a_{1}\right), \vec{x}\right), \vec{p}\right)$ e amostragem de $X_{2}^{\prime}$ com 0.85 para $X_{1}^{\prime}=1$ e 0.15 para $X_{2}^{\prime}=0$.

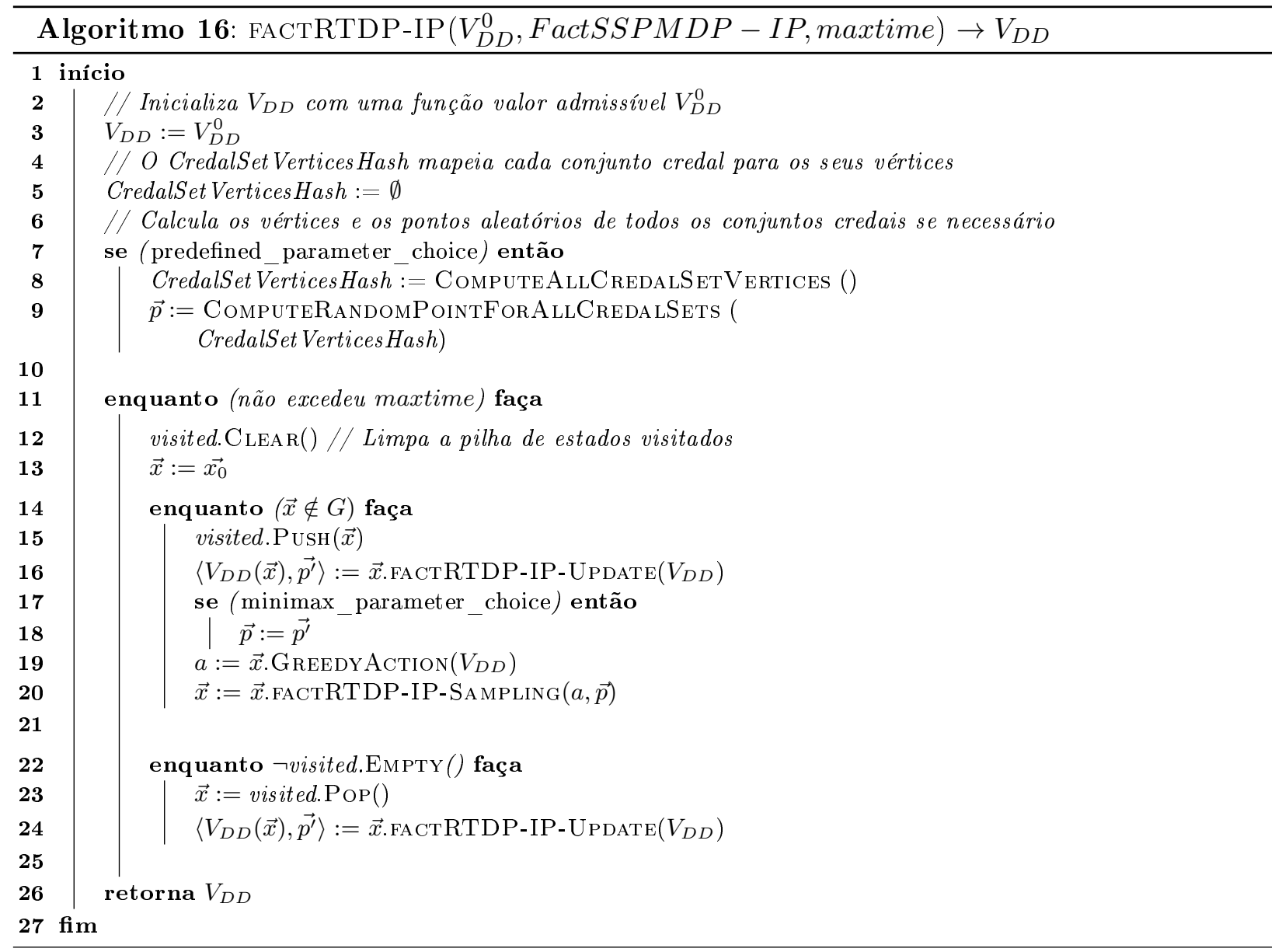

próximo estado é realizada (Algoritmo 16, Linha 20). Da Linha 22 a 25, o algoritmo atualiza todos os estados visitados em um trial em ordem reversa (do estado final ao estado inicial).

Já o algoritmo FACTRTDP-IP-UPDATE (Algoritmo 17) atualiza a função valor fatorada para 

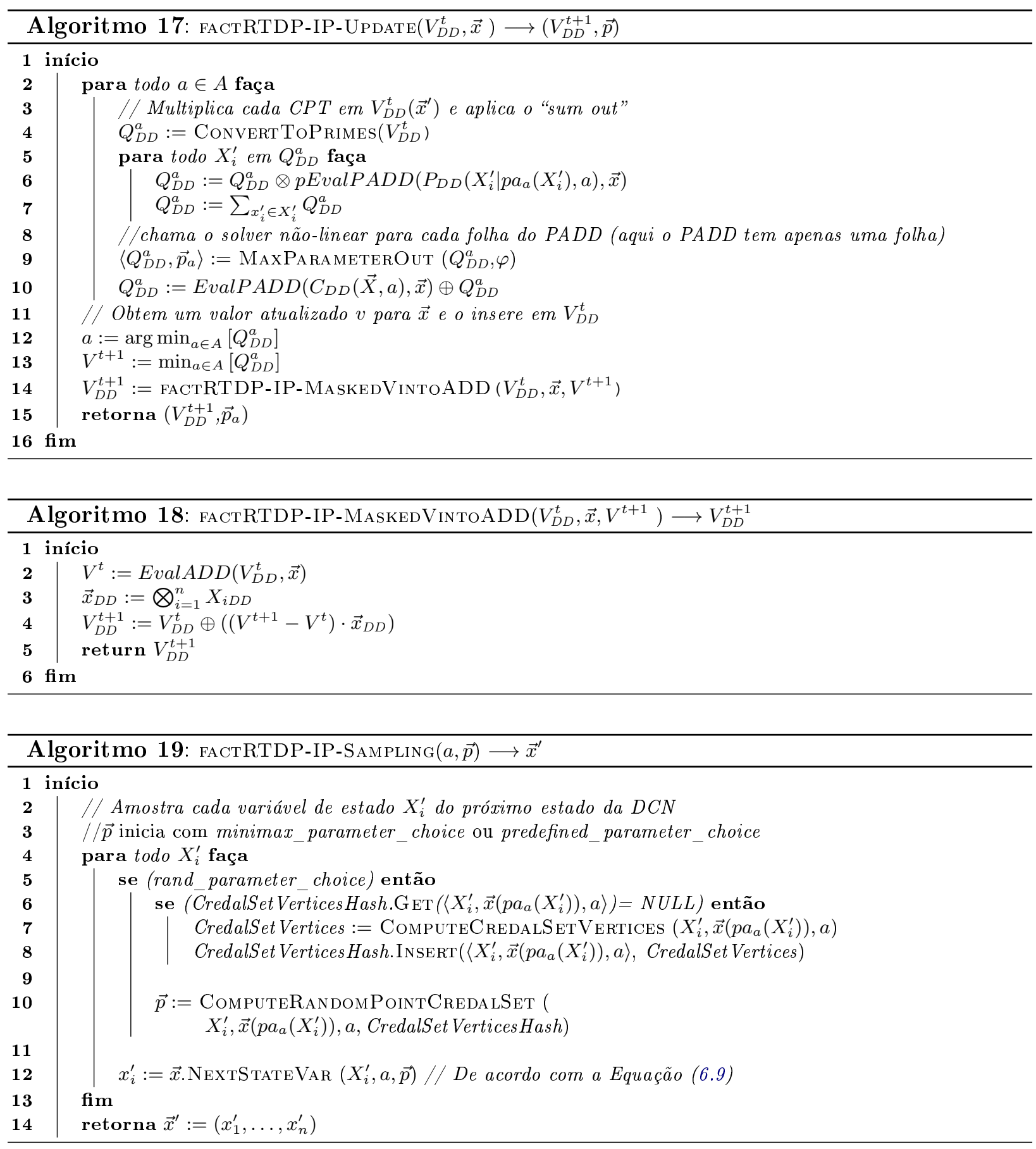

o estado $\vec{x}$ da seguinte forma: após colocar "linha" as variáveis de $V_{D D}^{t}$ (Algoritmo 17, Linha 4 , que converte cada $X_{i}$ em $\left.X_{i}^{\prime}\right), Q_{D D}^{a}$ é derivado através do cálculo da Equação (6.5) em termos de operações de PADD (Algoritmo 17, Linhas 5-7). Após aplicar a operação MaxParameterOut (Algoritmo 17, Linha 9) o resultado obtido é um valor real. O cálculo de $V^{t+1}(\vec{x})$ produz uma constante $V^{t+1}$ para um $\vec{x}$ conhecido (Algoritmo 17, Linha 13), então o factRTDP-IP insere esta nova constante $V^{t+1}$ na função valor atual $V_{D D}^{t}$ para obter $V_{D D}^{t+1}$ chamando o método FACTRTDPIP-MASKEDVINTOADD (Algoritmo 18).

O algoritmo FACTRTDP-IP-SAMPLing (Algoritmo 19) amostra o próximo estado $\vec{x}^{\prime}$ dado o estado atual $\vec{x}$, a ação $a$ e o parâmetro $\vec{p}$. Para escolher os valores para os parâmetros $\vec{p}$ um dos seguintes métodos é utilizado: minimax_parameter_choice, predefined_parameter_choice ou 
rand_parameter_choice.

Caso a escolha seja o método minimax_parameter_choice, $\vec{p}$ terá os mesmos valores que foram calculados para a ultima atualização. Caso seja o método predefined_parameter_choice, $\vec{p}$ é calculado somente uma vez no inicio do Algoritmo 16 (Linhas 7-9) calculado primeiro os vértices de todos os conjuntos credais (armazenados em uma tabela hash chamada CredalSetVerticesHash), e então utilizando estes vértices para gerar os pontos aleatórios. Por fim, caso o método rand_parameter_choice tenha sido escolhido, $\vec{p}$ é calculado para as tuplas $\left\langle X_{i}^{\prime}, \vec{x}\left(p a_{a}\left(X_{i}^{\prime}\right)\right), a\right\rangle$ com $i=\{1, \cdots, n\}$, onde $a$ é a ação gulosa atual e $\vec{x}\left(p a_{a}\left(X_{i}^{\prime}\right)\right)$ indica os valores dos pais de $X_{i}^{\prime}$ na atribuição de variáveis $\vec{x}$, i.e., a configuração dos pais de $X_{i}^{\prime}$ no estado atual $\vec{x}$ (Algoritmo 19 , Linhas 5-10). Note que o tamanho de CredalSetVerticesHash será o número de conjuntos credais para o método predefined_parameter_choice e será menor ou igual a este número para o método rand_parameter_choice considerando que nem todos os estados serão visitados. Assim, no pior caso o tamanho será igual a $|\mathcal{K}|_{\max }$ (Equação 5.3). Considerando que estão sendo utilizadas variáveis binárias e assumindo conjuntos credais fechados e convexos, o número de vértices para cada conjunto credal é igual a 2. Assim o espaço requerido pela tabela CredalSetVerticesHash é proporcional a uma constante vezes $|\mathcal{K}|_{\max }$.

Após definir os valores para $\vec{p}$ a amostragem é realizada para cada variável $X_{i}^{\prime}$ do próximo estado (Algoritmo 19, Linha 12). Isto é feito utilizando o ADD (da variável $X_{i}^{\prime}$ ) retornada por ValPADDLeaves $\left(p E v a l P A D D\left(P_{D D}\left(X_{i}^{\prime} \mid p a_{a}\left(X_{i}^{\prime}\right), a\right), \vec{x}\right), \vec{p}\right)$, em que estão as probabilidades para se realizar a amostragem.

\subsection{O Algoritmo factLRTDP-IP}

O algoritmo factLRTDP-IP (Algoritmo 20) é similar ao factRTDP-IP, com as seguintes diferenças: (i) na condição de parada do trial (Algoritmo 20, Linha 14), é verificado se o estado $\vec{x}$ está marcado como resolvido; (ii) no fim de cada trial (Algoritmo 20, Linhas 25 a 29) ao invés de realizar o FACTRTDP-IP-UPDATE em cada estado, é verificado se o estado pode ser rotulado como resolvido através do método FACTCHECKSOLVED-IP, que é semelhante ao CHECKSolVED (Algoritmo 6), com o uso do método FACtSucCessorStates-IP no lugar de SuccessorSTAtes. Visto que $\vec{x}$ é um estado fatorado no método faCtSuCCESSORStates-IP, é necessário encontrar o PADD de probabilidade conjunta para o estado $\vec{x}$ e a ação $a$ através da operação $\bigotimes_{i=1}^{n} p E v a l P A D D\left(P_{D D}\left(X_{i}^{\prime} \mid p a_{a}\left(X_{i}^{\prime}\right), a\right), \vec{x}\right)$ e então enumerar todos os caminhos que levam a uma folha que não seja 0 . Com estas operações, todos os estados sucessores de $\vec{x}$ são obtidos.

\subsection{Os Algoritmos SSiPP-IP e LSSiPP-IP}

Para adaptar os algoritmos SSiPP e LSSiPP (apresentados na Seção 3.2.3) para resolver SSP MDP-IPs foram feitas algumas alterações. Antes de apresenta-las, na Seção a seguir será mostrado como criar Short-Sighted SSP MDP-IPs a partir de SSP MDP-IPs.

\subsubsection{Short-Sighted SSP MDP-IPs}

Para se trabalhar com Short-Sighted SSP MDPs no contexto de probabilidades imprecisas, a métrica que define um Short-Sighted SSP MDP (Equação 2.8) foi adaptada para considerar todos 


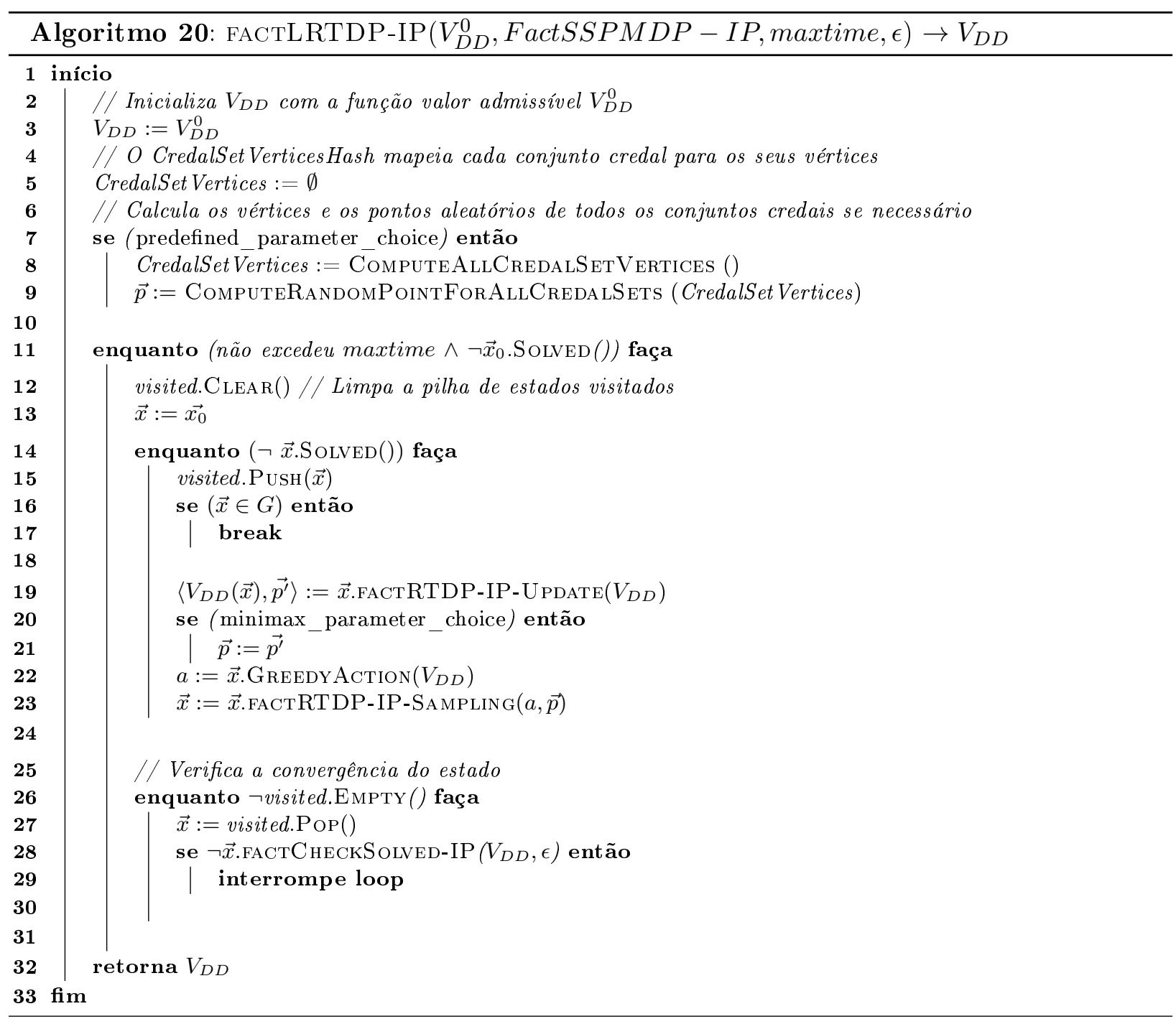

os estados sucessores de um dado estado $s$ dado qualquer distribuição de probabilidade $P$ tal que $P \in K(\cdot \mid s, a)$ para todo $a \in A$. Assim, a métrica agora é definida por:

$$
\delta_{I P}\left(s, s^{\prime}\right)= \begin{cases}0 & \text { se } s=s^{\prime} \\ 1+\min _{a \in A} \min _{\hat{s}: P(\hat{s} \mid s, a)>0 \forall P \in K(\cdot \mid s, a)} \delta_{I P}\left(\hat{s}, s^{\prime}\right) & \text { caso contrário }\end{cases}
$$

Esta métrica continua representando a distância não simétrica "em ações" de $s$ a $s^{\prime}$.

\section{Definição 6.5.1. (Short-Sighted Stochastic Shortest Path MDPs com probabilidades imprecisas (Short-Sighted SSP MDP-IP))}

Dado um SSP MDP-IP definido pela tupla $\mathcal{S}=\left\{S, A, C, \mathcal{K}, G, s_{0}\right\}$ é possivel ter um ShortSighted SSP MDP-IP centrado em um estado $s \in S$ e com profundidade $t \in N^{*}$ definido pela tupla $\mathcal{S}_{s, t}=\left\{S_{s, t}, A, C_{s, t}, \mathcal{K}, G_{s, t}, s\right\}$, em que:

- $S_{s, t}$ e $G_{s, t}$ são respectivamente o conjunto de estados resultantes da poda e o conjunto de estados meta artificiais, definidos em função de $\delta_{I P}$ como:

$$
S_{s, t}=\left\{s^{\prime} \in S \mid \delta_{I P}\left(s, s^{\prime}\right) \leq t\right\}
$$




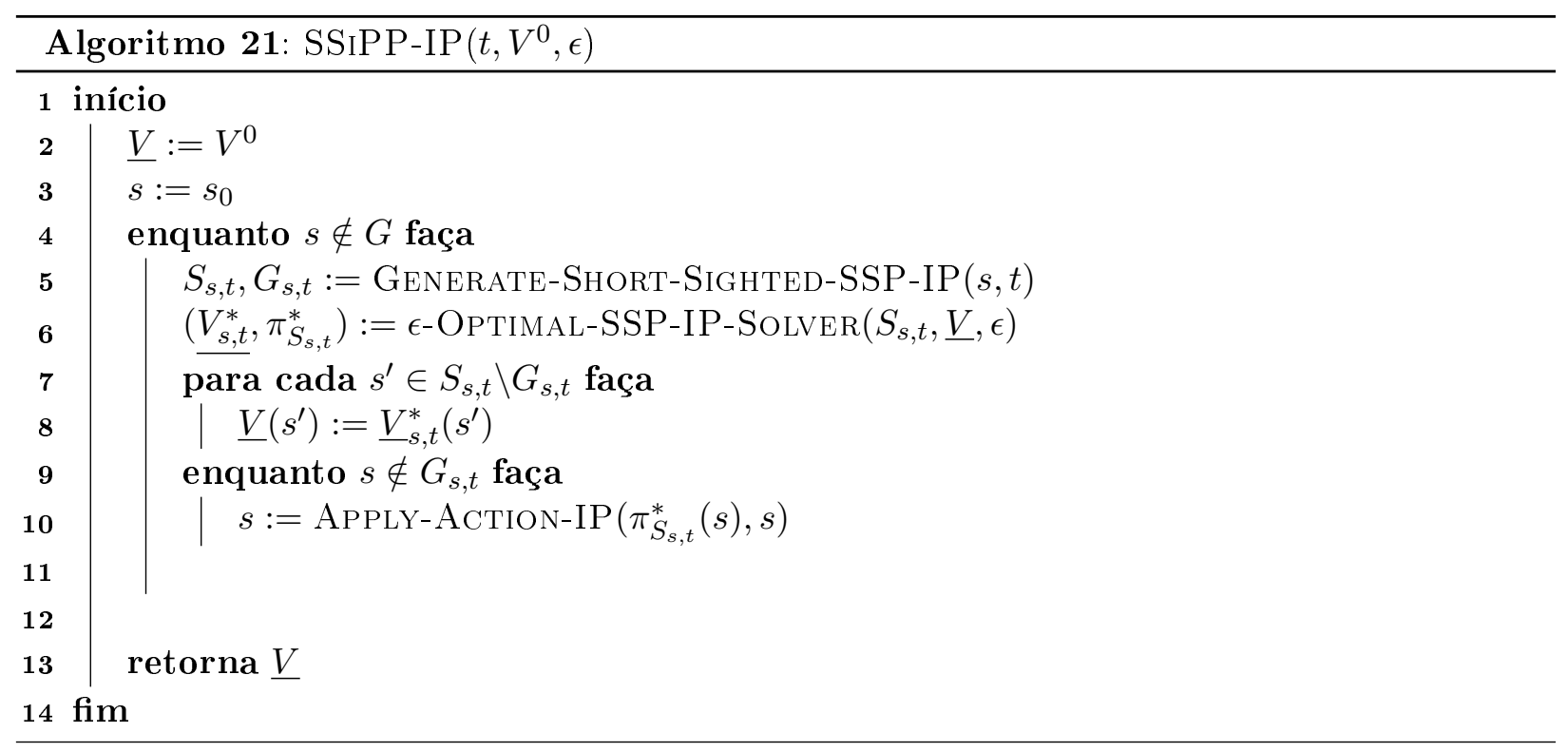

$$
G_{s, t}=\left\{s^{\prime} \in S \mid \delta_{I P}\left(s, s^{\prime}\right)=t\right\} \cup\left(G \cap S_{s, t}\right)
$$

- $C_{s, t}\left(s^{\prime}, a, s^{\prime \prime}\right)$ e $H$ seguem a mesma definição dos seus respectivos pares em um Short-Sighted SSP MDP. Assim como no Short-Sighted SSP MDP, neste trabalho será considerado que o custo depende apenas de $s$ e a, i.e., $C_{s, t}(s, a)$ e $H\left(s^{\prime \prime}\right)=0$.

Para gerar Short-Sighted SSP MDP-IPs o algoritmo GenerAte-ShorT-Sighted-SSP, agora chamado Generate-ShorT-Sighted-SSP-IP foi alterado considerando a distância $\delta_{I P}$ para gerar um subproblema. Além disso, o método que gera os estados sucessores (SucCessorStates), utilizado por ele para gerar os estados sucessores foi alterado para considerar todas as transições maiores que zero ou com parâmetros. Este método agora é chamado de SucCessorStATES-IP.

\subsubsection{SSiPP-IP e LSSiPP-IP}

O algoritmo SSiPP-IP (Algoritmo 21) chama o método Generate-ShorT-Sighted-SSP-IP (Algoritmo 21, Linha 5) para gerar um Short-Sighted SSP MDP-IP e chama um planejador $\epsilon$-ótimo externo para resolver-lo, através do método $\epsilon$-Optimal-SSP-IP-Solver (Algoritmo 21, Linha 6). Além disso o método Apply-ACtion-IP (Algoritmo 21, Linha 10) é chamado para simular a execução de uma ação. Este método necessita amostrar um próximo estado usando esta ação. Assim como no factRTDP-IP este método precisa considerar um dos métodos apresentados na Seção 6.1 para escolher o valor dos parâmetros de probabilidade.

No algoritmo Labeled SSiPP-IP (LSSiPP-IP, Algoritmo 22) foi feita adicionalmente uma mudança. Nesse algoritmo é chamado agora o CheckSolved-IP (Algoritmo 22, Linha 21). 


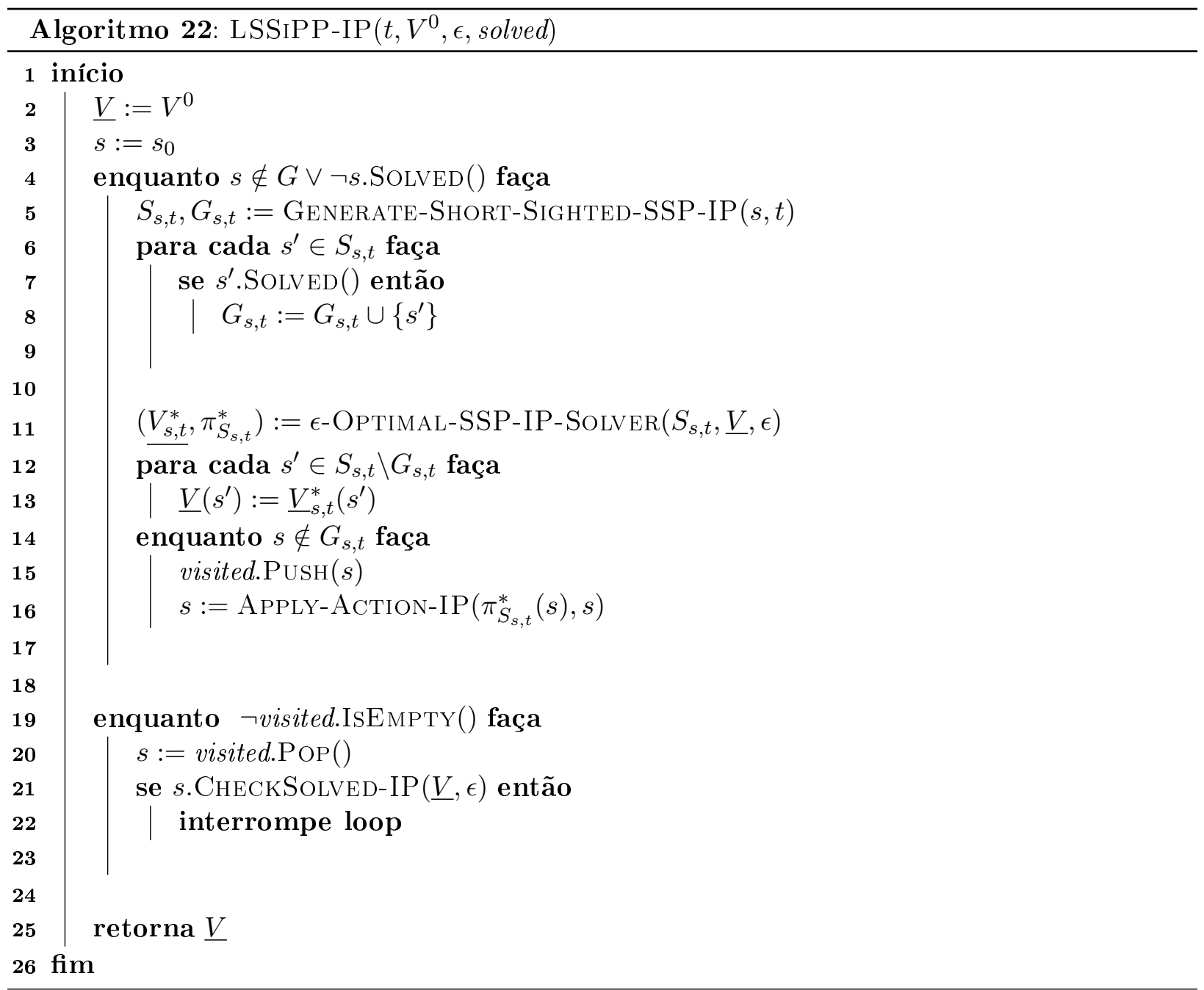




\section{Capítulo 7}

\section{Experimentos}

Neste capítulo, a análise empírica dos algoritmos propostos será dividida em duas partes. Na primeira parte será realizada uma comparação entre os algoritmos assíncronos propostos, RTDPIP, o LRTDP-IP, o factRTDP-IP e o factLRTDP-IP com o algoritmo SPUDD-IP (Delgado et al., 2011), que é um algoritmo síncrono que calcula políticas ótimas (para mais detalhes ver a Seção $5.2 .2)$.

Na segunda parte, uma comparação entre os algoritmos LRTDP-IP e LSSiPP-IP será realizada.

\subsection{Domínios}

Os algoritmos foram executados em três domínios de planejamento bem conhecidos: o SysAdmin, proposto por Guestrin et.al. (2003); Navigation e Triangle Tireworld, ambos do International Probabilistic Planning Competition (IPPC).

Para gerar problemas SSP MDP-IPs a partir destes domínios, os mesmos foram modificados adicionando parâmetros na função de transição de probabilidades e um conjunto de restrições como descritos a seguir.

\subsubsection{Domínio Navigation}

O domínio Navigation foi extraído do IPPC-2011. Nesse domínio um robô deve se movimentar em um grid de $n \times m$ posições e encontrar um caminho para alcançar uma posição destino, evitando posições "perigosas", que podem fazer o robô desaparecer com determinada probabilidade. O robô pode andar uma posição por vez em uma das quatro direções (norte, sul, leste e oeste) ou pode se manter no mesmo lugar (noop). O problema é representado de forma fatorada com $n \times m$ variáveis, onde cada variável representa se o robô está em uma determinada posição do grid. A ação de andar para uma posição é determinística, porém existe uma probabilidade do robô desaparecer dada pelo parâmetro $p_{i}$ sujeito a $b_{j} \leq p_{i} \leq b_{k}+0.1$, em que $b_{j}$ e $b_{k}$ são valores limites que variam de acordo com a coluna do grid, em que quanto mais próxima a coluna estiver da posição destino, maiores serão estes valores, indicando que, quanto mais próximo do destino, maior a probabilidade do robô desaparecer. O robô tem custo 0 (zero) caso esteja na posição destino ou 1 caso esteja em qualquer outra posição do grid. Este é um problema esparso, visto que para qualquer ação, o número de estados alcançáveis é uma constante para todas as instâncias. 


\subsubsection{Domínio Triangle Tireworld}

O domínio Triangle Tireworld (Little e Thiébaux, 2007) é baseado no domínio utilizado no IPPC-2005, que tem como idéia controlar a movimentação de um carro, que deve ir de uma localização inicial a uma localização de destino escolhendo uma série de estradas. Cada estrada conecta duas localizações. Neste problema, ao trafegar em uma estrada, o pneu do carro tem uma determinada probabilidade de furar. Nesta situação o agente pode trocar o pneu furado por um estepe. Além disso, ao se trafegar por certas localizações, caso o carro não tenha estepe reserva, o agente pode recarregá-lo uma vez para usar em situações futuras caso a localização tenha estepe (essa versão do domínio é chamada de NoRelaxed Triangle Tireworld). A representação fatorada neste problema se dá por uma variável para cada possível localização do carro no mapa, uma variável indicando se há estepe em uma localização, uma variável indicando se o carro tem um pneu furado e uma variável indicando se o carro tem pneu reserva. A probabilidade de um pneu do carro furar é dada pela restrição $b_{j} \leq p_{i} \leq b_{k}+0.1$, em que $b_{j}$ e $b_{k}$ são valores limites que variam de acordo com a posição no mapa, quanto mais próxima a localização estiver do destino, maiores serão estes valores, indicando que quanto mais próximo do destino, maior a probabilidade do pneu furar. O agente tem custo 0 (zero) caso esteja na localização destino ou 1 caso esteja em qualquer outra localização.

O domínio Relaxed Triangle Tireworld é uma ligeira variação do domínio NoRelaxed Triangle Tireworld, em que o carro pode recarregar o estepe mais de uma vez em uma mesma localização. Com isto não há necessidade de ter variáveis para representar se o estepe único existe em uma determinada localização ou não, o que diminui a quantidade de variáveis, removendo cerca de $30 \%$ das variáveis do problema original.

Este domínio também é considerado esparso, i.e., o número de estados alcançáveis em um passo é uma constante.

\subsubsection{Domínio SysAdmin}

No domínio SysAdmin (Guestrin et al., 2003) existem $n$ computadores $c_{1}, \ldots, c_{n}$ conectados entre si com a topologia de um anel unidirecional. Neste domínio exitem $n+1$ ações que pode ser executadas: reboot $\left(c_{1}\right), \ldots$, reboot $\left(c_{n}\right)$ e notreboot (i.e. nenhuma máquina precisa ser reiniciada). Os parâmetros de probabilidade $p_{i 1}$ e $p_{i 2}$ são utilizados para definir a probabilidade de cada computador $i$ manter-se funcionando (ou entrar em estado de falha) no próximo estado. Estes parâmetros têm a seguintes restrição: $0.85+p_{i}^{\prime} \leq p_{i} \leq 0.95$. Neste problema não existe estado meta, assim ele é um problema MDP-IP de horizonte infinito. Ele pode ser considerado complexo, visto que é um domínio denso e com muitas transições com probabilidades imprecisas. Para converter este problema em um SSP MDP-IP, o método de conversão descrito em (Bertsekas e Tsitsiklis, 1996) foi utilizado. Dado o fator de desconto $\gamma=0.9$, um estado meta que é alcançado por todo estado do problema com probabilidade $1-\gamma$ foi adicionado no problema. No estado inicial, o problema começa com metade dos computadores rodando, o custo é 0 para o estado meta e $C(\vec{x})=\sum_{i=1}^{n} x_{i}$ para os outros estados.

\subsection{Configuração dos Experimentos}

Para todos os experimentos foi utilizada uma máquina virtual Linux que contém dois processadores de $3.4 \mathrm{GHz}, 5$ GB de memória com o sistema operacional Ubuntu 12.10 e o ambiente Java 6 
1. Para as análises, o SPUDD-IP, LRTDP-IP, o factLRTDP-IP e o LSSiPP-IP utilizam o erro de convergência $\epsilon=0.01$ para todos os domínios. Os algoritmos que não conseguiram executar uma instância do problema em duas horas ou excederam o uso de memória foram marcados como DNF (do inglês, Did Not Finish). Em todas as análises foi usada a função valor inicial $V^{0}=\min _{s \in S} C(s)=0$. Para o cálculo da maximização dos parâmetros no Bellman Backup de todos os algoritmos, o solver MINOS (Murtagh e Saunders, 1978) é utilizado.

A identificação de deadends foi implementada em todos os algoritmos. Nos domínios Navigation e Triangle Tireworld um deadend é um estado onde nenhuma ação está disponível (i.e. qualquer ação aplicada leva sempre ao mesmo estado). Nos algoritmos assíncronos, caso um deadend seja encontrado, sua busca será interrompida (execução de um trial no caso dos algoritmos baseados no RTDP e execução de uma rodada no caso dos algoritmos baseados no SSiPP) e o estado terá o valor atualizado para $\infty$. No CheckSolved-IP, se um deadend é encontrado, o valor dele também é atualizado para $\infty$ e o mesmo é ignorado nas buscas do algoritmo.

A análise dos algoritmos em ambos os casos foi realizada em termos de: taxa de convergência do estado inicial e tempo de convergência para os estados. Os algoritmos também foram analisados em relação ao número de chamadas ao solver multilinear na resolução dos problemas.

\subsection{Comparação entre SPUDD-IP, RTDP-IP, LRTDP-IP, factRTDP- IP e factLRTDP-IP}

\subsubsection{Taxa de Convergência do Estado Inicial}

Para analisar o comportamento de convergência do SPUDD-IP, RTDP-IP, LRTDP-IP, factRTDP$I P$ e factLRTDP-IP, o valor do estado inicial $s_{0}$ é observado durante o tempo até alcançar a convergência para três problemas de planejamento: a instância 18 do domínio Navigation, a instância 17 do domínio Relaxed Triangle Tireworld e a instância 6 do domínio SysAdmin ${ }^{2}$. Cada um destes problemas corresponde à maior instância resolvida em cada domínio pelos cinco algoritmos (como será visto na Seção 7.3.2).

Os algoritmos foram executados considerando os três diferentes métodos de se escolher a probabilidade de amostrar o próximo estado, i.e. os métodos minimax_parameter_choice, predefined_parameter_choice e rand_parameter_choice. Intuitivamente, era esperado que o método minimax_parameter_choice auxiliasse $V\left(s_{0}\right)$ a convergir mais rápido. Entretanto, a convergência de $V\left(s_{0}\right)$ se manteve semelhante para os três métodos. Nesta seção apenas os resultados para o método minimax_parameter_choice são mostrados, enquanto a comparação dos três métodos em termos de tempo será realizada na próxima seção.

Na Figura 7.1 é possível ver que para os domínios esparsos (Navigation e Relaxed Triangle Tireworld), os algoritmos assíncronos (e.g. com o método minimax_parameter_choice) foram muito melhor que o SPUDD-IP. Na instância do domínio Navigation pode-se observar que o RTDP-IP, $L R T D P$-IP , factRTDP-IP e o factLRTDP-IP alcançam um valor próximo do ótimo rapidamente, nos primeiros 4 segundos, enquanto o SPUDD-IP leva mais de 5000 segundos. Um comportamento similar pode ser visto para a instância do Relaxed Triangle Tireworld.

\footnotetext{
${ }^{1}$ Os fontes utilizados para a execução dos algoritmos estão disponíveis em https://code.google.com/p/mdp-ip/

${ }^{2} \mathrm{O}$ número da instância representa o número de variáveis em um problema, assim o número de estados do problema é $2^{n}$ onde $n$ é o número de variáveis.
} 

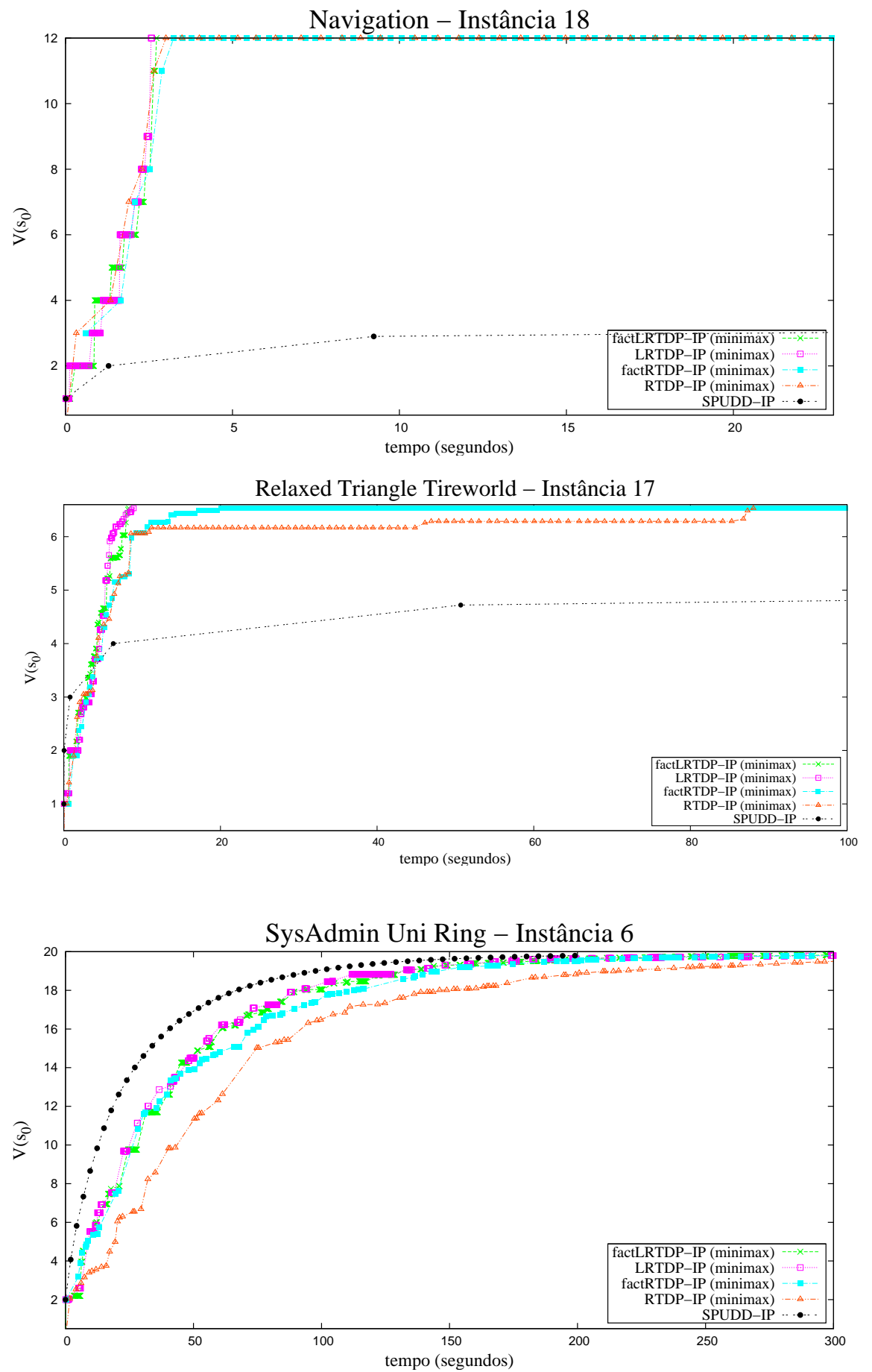

Figura 7.1: Valor do estado inicial para as instâncias 18 do dominio Navigation, 17 do domínio Relaxed Triangle Tireworld e 6 do dominio SysAdmin para os algoritmos SPUDD-IP, RTDP-IP, LRTDP-IP, factRTDP-IP e factLRTDP-IP. 
Entretanto, para a instância do domínio SysAdmin (Figura 7.1, parte de baixo), que é um domínio denso, o SPUDD-IP tem uma taxa de convergência melhor do que a dos algoritmos assíncronos, RTDP-IP, LRTDP-IP, factRTDP-IP $e$ factLRTDP-IP. Note que neste domínio, devido a eventos exógenos, todos os estados são alcançáveis e assim os algoritmos assíncronos não tem nenhum ganho de conhecer o estado inicial. Neste caso, os algoritmos assíncronos gastam mais tempo atualizando estado por estado que o SPUDD-IP, que atualiza todos os estados em cada passo. Também, com o intuito de rotular estados resolvidos, o LRTDP-IP e o factLRTDP-IP exploram a estrutura de grafo guloso enraizado a partir do estado inicial, operação que pode ser muito custosa quando o grafo é denso. Nesta situação, o método CHeCkSolved apenas desperdiça tempo visto que todos os estados do problema irão convergir juntos. Outro detalhe a ser observado é o fato que, devido ao SPUDD-IP realizar as atualizações de forma síncrona, $V\left(s_{0}\right)$ é alterado de forma uniforme a cada iteração (veja a curva do SPUDD-IP na Figura 7.1), enquanto no caso da programação dinâmica assíncrona, o valor de $s_{0}$ pode não mudar durante certos intervalos de tempo (dependendo de quais estados foram atualizados durante os trials).

Como o LRTDP-IP e o factLRTDP-IP convergiram mais rápido que o RTDP-IP e o factRTDP$I P$ para todos os domínios e instâncias, nas próximas sub-seções serão apenas comparados os resultados do LRTDP-IP e do factLRTDP-IP com os do SPUDD-IP.

\subsubsection{Tempo de Convergência por Instância de Problema}

A Figura 7.2 mostra o tempo de convergência global do SPUDD-IP, do LRTDP-IP e do factLRTDP-IP para diferentes instâncias dos domínios Navigation, Relaxed Triangle Tireworld e SysAdmin. Nela também é mostrada a comparação entre minimax_parameter_choice (abreviado como minimax), predefined_parameter_choice (pre) e rand_parameter_choice (rand).

Para dominios esparsos como o Navigation e Relaxed Triangle Tireworld, as soluçôes assíncronas LRTDP-IP $e$ factLRTDP-IP mostram uma melhora de até três ordens de magnitude em relação a solução de programação dinâmica síncrona SPUDD-IP. Adicionalmente, enquanto o SPUDD-IP não é capaz de retornar políticas para instâncias com mais de 20 variáveis, $o$ LRTDP-IP $e$ factLRTDPIP retornam políticas ótimas para instâncias com até 121 variáveis no domínio Navigation e com até 80 variáveis no domínio Relaxed Triangle Tireworld. Como esperado, isto ocorre porque nestes domínios eles realizam Bellman backups somente no conjunto de estados alcançáveis a partir do estado inicial, enquanto o SPUDD-IP precisa atualizar todos os estados em cada iteração. O LRTDPIP tem uma melhora de convergência em relação ao factLRTDP-IP de 2 vezes, o que não é uma diferença significante.

Em todos os gráficos da Figura 7.2, o tempo de convergência do factLRTDP-IP é similar para os três métodos. Uma explicação para este fato é que nos três domínios analisados, $\max _{a, X_{i}}\left|p a_{a}\left(X_{i}\right)\right|=$ 2 ou $3 ;|K| \leq|K|_{\max }=n \times 2^{3} \times|A|$ (Equação 5.3) e o número de vértices de cada conjunto credal é no máximo 2 (devido ao fato de se estar trabalhando com variáveis booleanas). Assim, não existe custo adicional significativo para calcular os vértices do conjunto credal e para gerar pontos aleatórios utilizando a Equação 6.1 para os métodos predefined_parameter_choice e rand_parameter_choice, o que não causa diferença no tempo de amostragem em relação aos métodos minimax_parameter_choice, predefined_parameter_choice e rand_parameter_choice. Para domínios com uma grande dependência de variáveis e um grande número de vértices para os conjuntos credais é possível que rand_parameter_choice apresente um pior desempenho. 
Já em domínios densos como o SysAdmin, os resultados não são promissores. Nele pode-se ver que o LRTDP-IP e o factLRTDP-IP levam mais tempo para convergir (aproximadamente $4 \mathrm{x}$ mais) em comparação ao SPUDD-IP. Como já mencionado na seção anterior, isto se deve ao fato de, em um domínio denso, a convergência depender do valor de todos os estados. Desta forma os algoritmos de programação dinâmica síncronas se saem melhor neste tipo de domínio.

\subsubsection{Número de Chamadas ao Solver Multilinear}

Para mostrar a relação entre o número de chamadas do solver multilinear e o tempo de convergência dos problemas foi feita uma comparação entre o SPUDD-IP, o LRTDP-IP e o factLRTDP-IP com o método minimax_parameter_choice para escolher valores para cada $p_{i}$ em diferentes instâncias do domínio Relaxed Triangle Tireworld.

A Figura 7.3 mostra que para cada uma das abordagens analisadas existe uma forte correlação entre o tempo de convergência e as chamadas do solver multilinear, indicando que o número de chamadas ao solver domina o tempo de execução do algoritmo. Também pode-se notar que o número de chamadas ao solver multilinear para o SPUDD-IP cresce mais rápido que o número de chamadas ao solver multilinear para o $L R T D P$-IP e o factLRTDP-IP. Isto se deve ao fato de o SPUDD-IP atualizar todos os estados enquanto o LRTDP-IP e o factLRTDP-IP atualizam somente os estados alcançáveis, e o número de chamadas ao solver multilinear ser proporcional ao número de Bellman updates realizados.

\subsection{Comparação entre o LRTDP-IP e o LSSiPP-IP}

Uma vez que nos experimentos anteriores o LRTDP-IP teve um melhor desempenho em domínios esparsos, nestes experimentos o LSSiPP-IP será comparado apenas com o LRTDP-IP em três domínios deste tipo: Navigation, NoRelaxed Triangle Tireworld e Relaxed Triangle Tireworld. A comparação será feita em termos de comportamento de convergência do estado inicial, de tempo de convergência e de número de chamadas ao solver multilinear. O LSSiPP-IP foi executado considerando três valores de profundidade $t: 1,3$ e 5 e usando como planejador externo o LRTDP-IP.

Na Figura 7.4 é mostrado o valor de convergência do estado inicial ao longo do tempo para as instâncias 18 do Navigation, 18 do NoRelaxed Triangle Tireworld e 17 do Relaxed Triangle Tireworld. O valor do estado inicial usando o LRTDP-IP converge mais rápido para todos os problemas que o LSSiPP-IP.

Na Figura 7.5 é mostrado o tempo de convergência dos algoritmos. Nos três domínios o LRTDPIP é melhor resolvendo os problemas mais rápido enquanto as diferentes configurações do LSSiPPIP tem desempenho variável, sendo a configuração $t=1$ melhor no domínio Navigation, $t=3$ no Relaxed Triangle Tireworld e $t=5$ no NoRelaxed Triangle Tireworld.

Na Figura 7.6, percebe-se também que existe uma correlação entre o tempo de execução do algoritmo e o número de chamadas ao solver multilinear, com o LRTDP-IP fazendo menos chamadas que todas as configurações do LSSiPP-IP.

Nos experimentos para SSP MDPs realizados em Trevizan (2013), o LSSiPP apresenta um melhor desempenho que o LRTDP em alguns domínios. Porém o comportamento não é o mesmo para SSP MDP-IPs. Um possível motivo é que o LSSiPP-IP pode realizar mais chamadas ao solver para resolver o problema (como pode ser observado nas Figura 7.6) o que pode indicar que a 

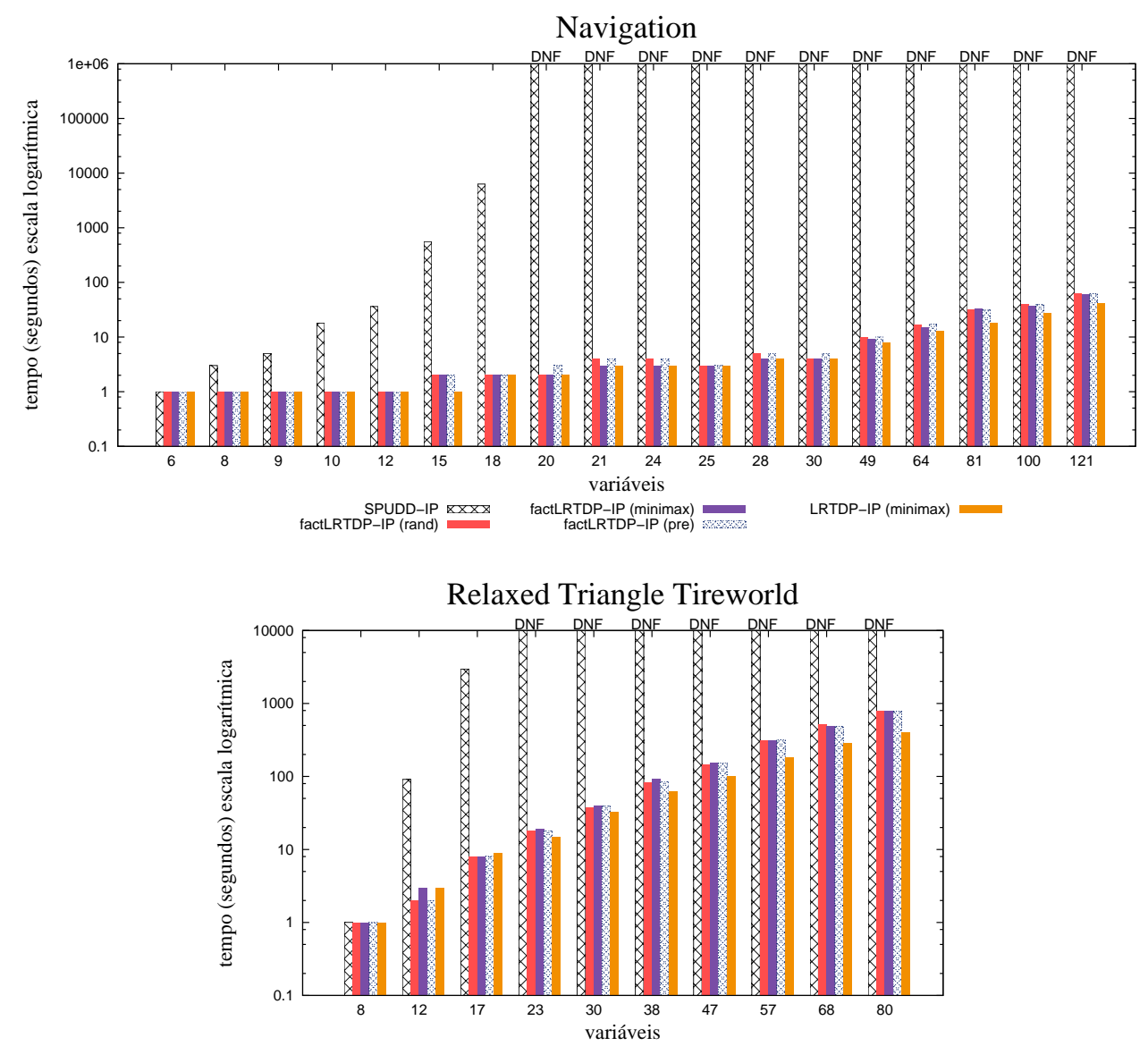

SactLRPUDD-IP
XXXX
factLRTDP-IP (minimax)

SysAdmin Uni Ring

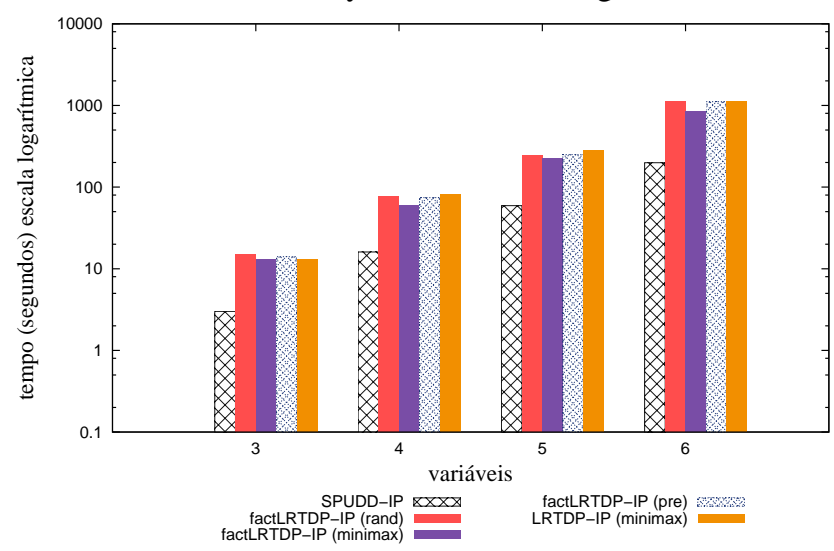

Figura 7.2: Tempo de convergência do SPUDD-IP, LRTDP-IP e o factLRTDP-IP em escala logarítmica, para os domínios Navigation, Relaxed Triangle Tireworld e SysAdmin. 


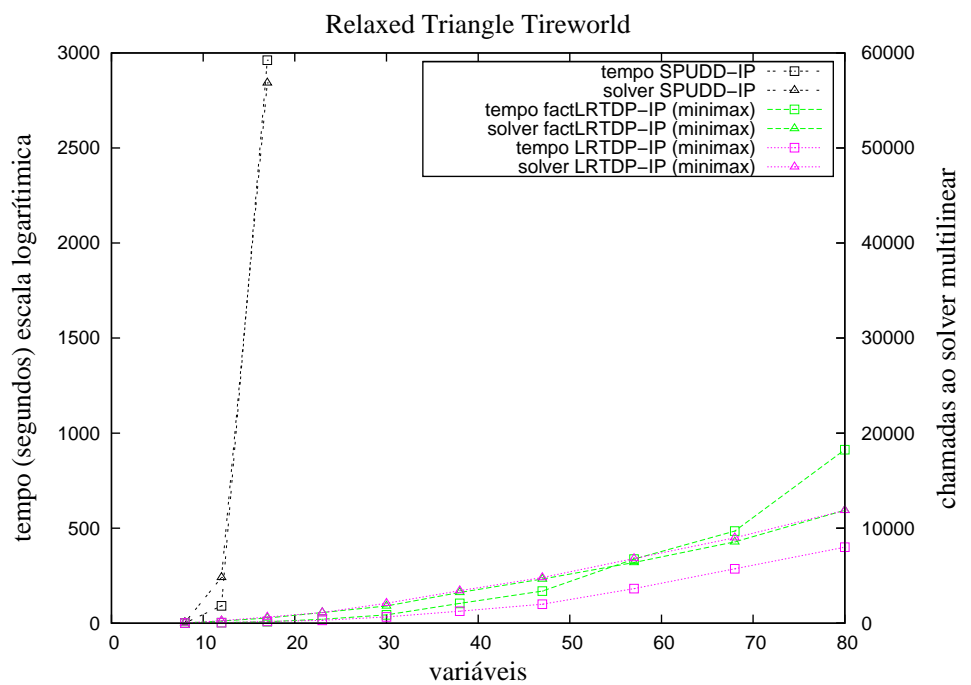

Figura 7.3: Tempo de convergência e número de chamadas ao solver multilinear para os algoritmos SPUDDIP, o LRTDP-IP e o factLRTDP-IP com o método minimax_parameter_choice.

estratégia de subdividir um SSP MDP-IP em vários subproblemas e resolve-los pode não ser tão vantajoso quanto resolver o problema como um todo. Na Figura 7.7, pode-se ver que o tempo de chamada ao solver do LSSiPP-IP $(t=5)$ corresponde a quase todo o tempo de execução do LRTDPIP, para o LSSiPP-IP o tempo do solver representa aproximadamente $60 \%$ do tempo total de execução do algoritmo, o que indica que não são apenas as chamadas do solver que são custosas no algoritmo. Outro ponto a ser observado é que foi usada uma função inicial muito simples. O projeto de melhores funções valor iniciais, de diminuição das chamadas ao solver e demais melhorias ao LSSiPP-IP serão deixados para trabalhos futuros. 

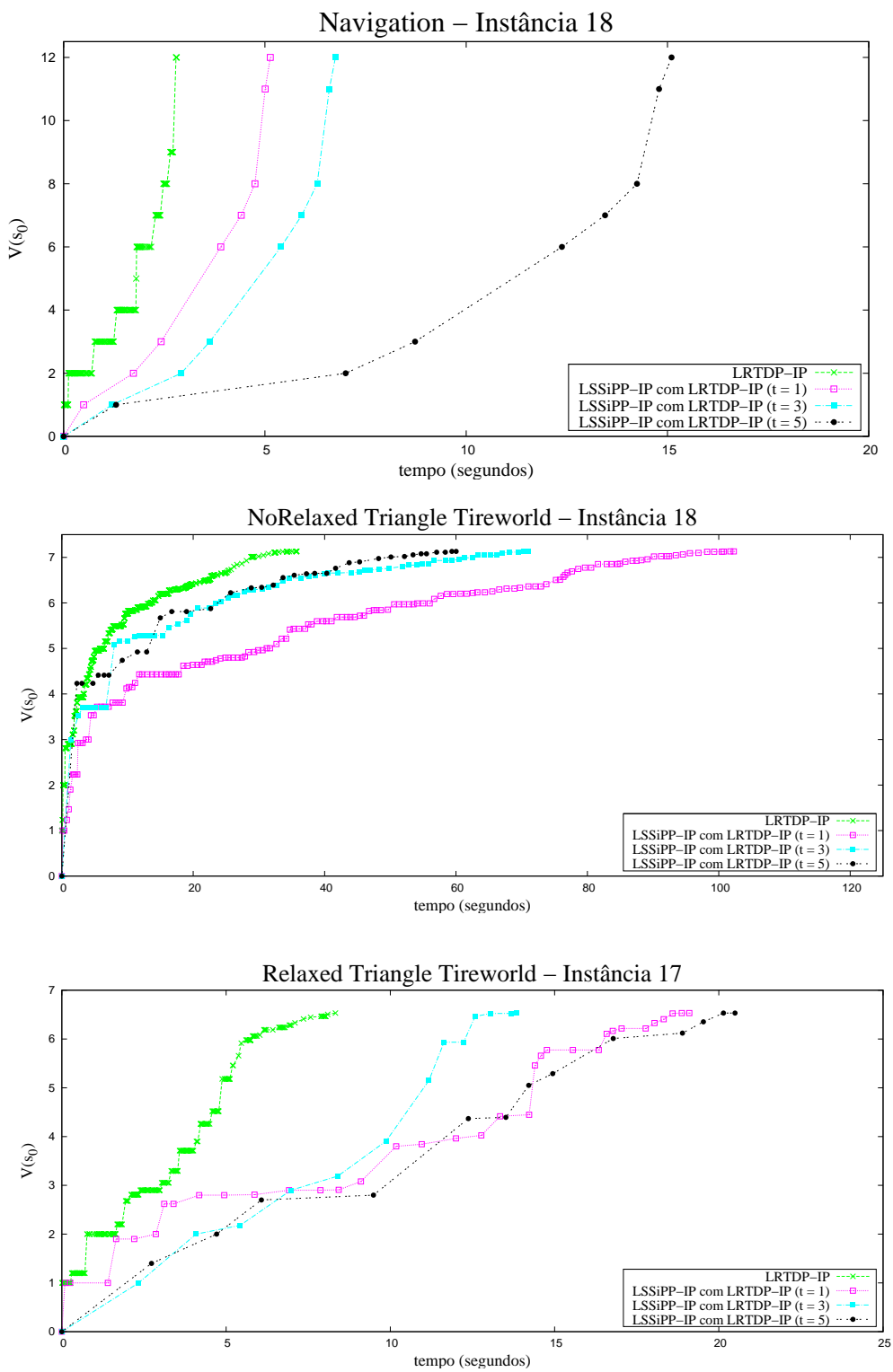

Figura 7.4: Valor do estado inicial para as instâncias 18 do Navigation, 18 do NoRelaxed Triangle Tireworld e 17 do Relaxed Triangle Tireworld para os algoritmos do LSSiPP-IP e LRTDP-IP. 

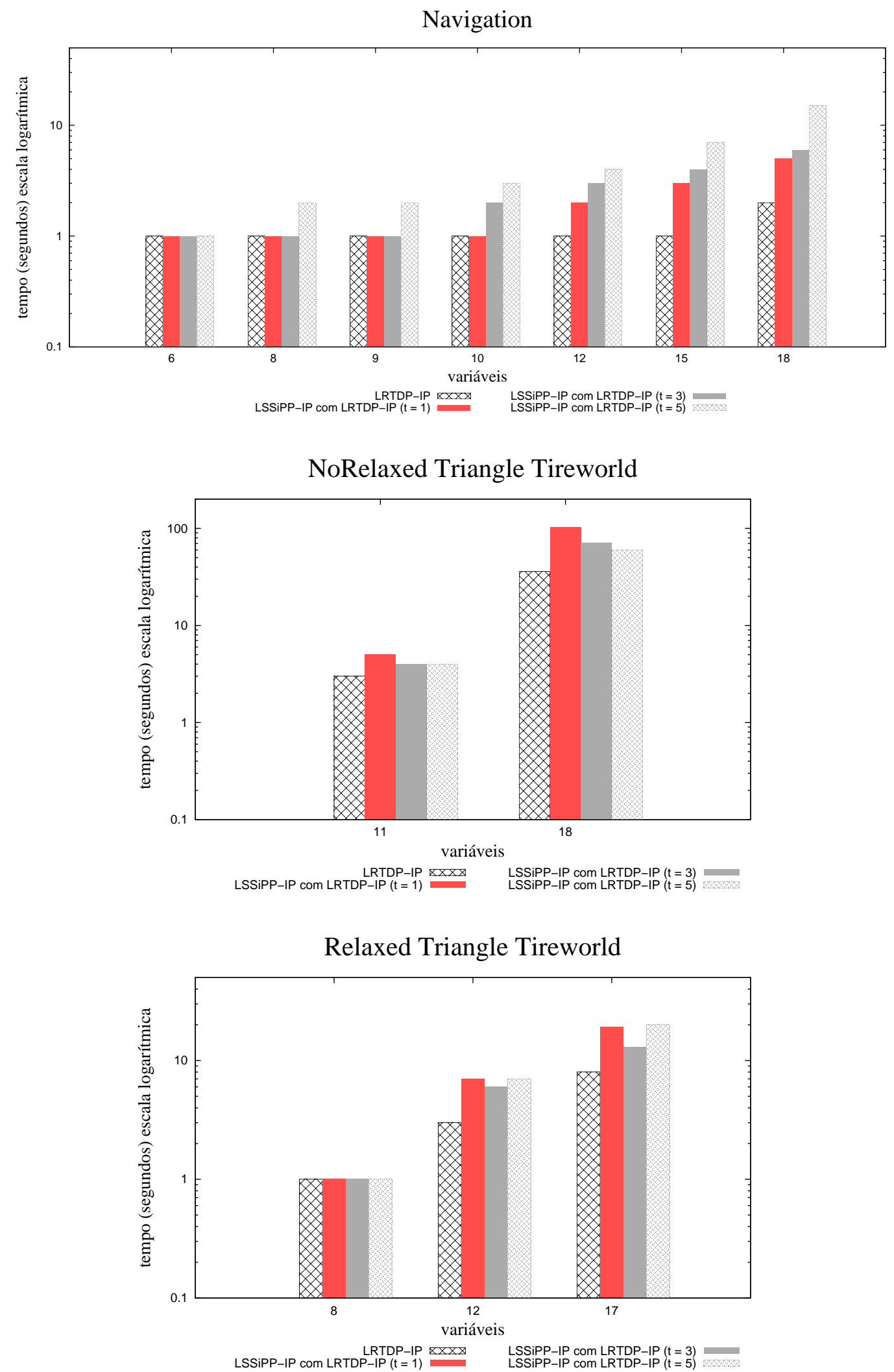

Figura 7.5: Tempo de convergência do LSSiPP-IP e LRTDP-IP em escala logarítmica para os domínios Navigation, NoRelaxed Triangle Tireworld e Relaxed Triangle Tireworld. 

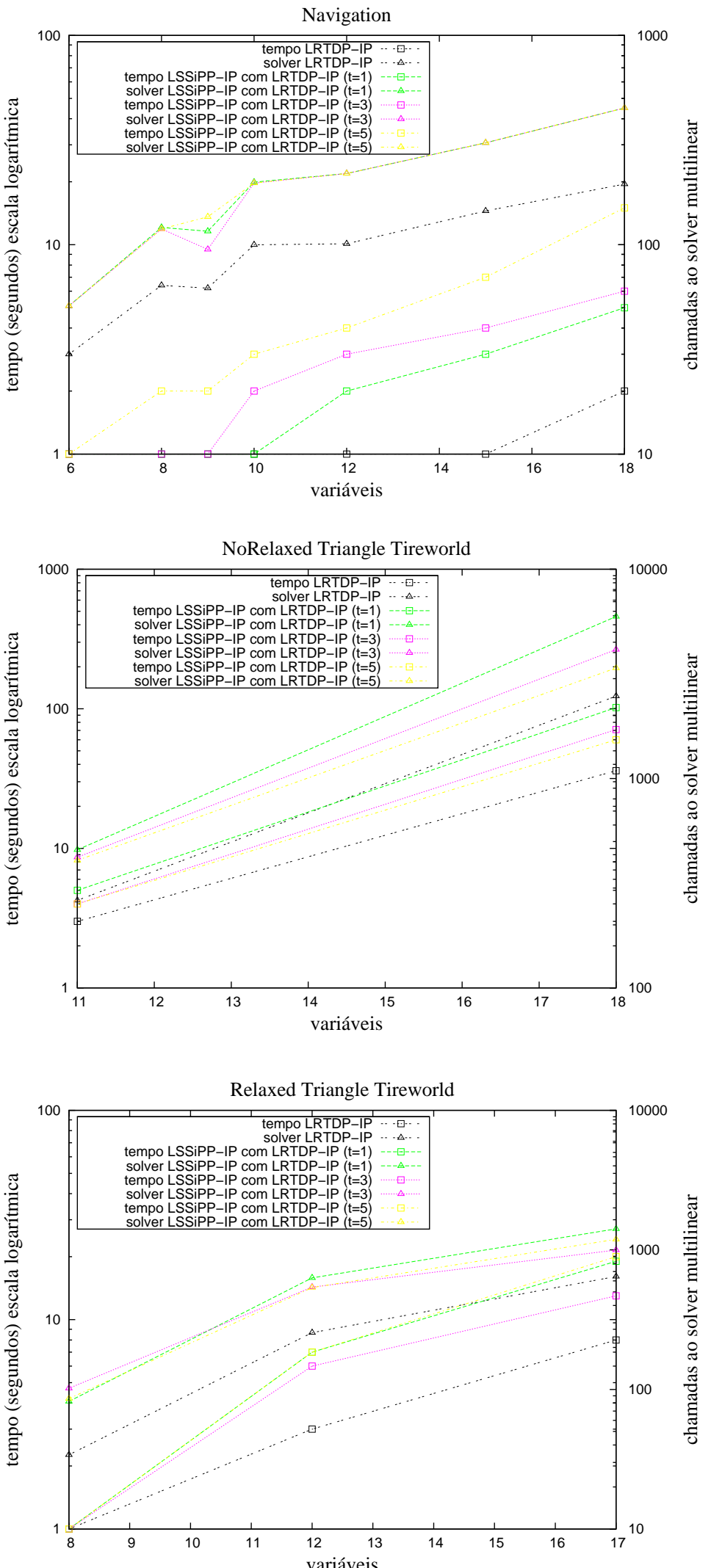

Figura 7.6: Tempo de convergência e nümero de chamadas ao solver multilinear para os algoritmos LSSiPPIP e LRTDP-IP. 
NoRelaxed Triangle Tireworld

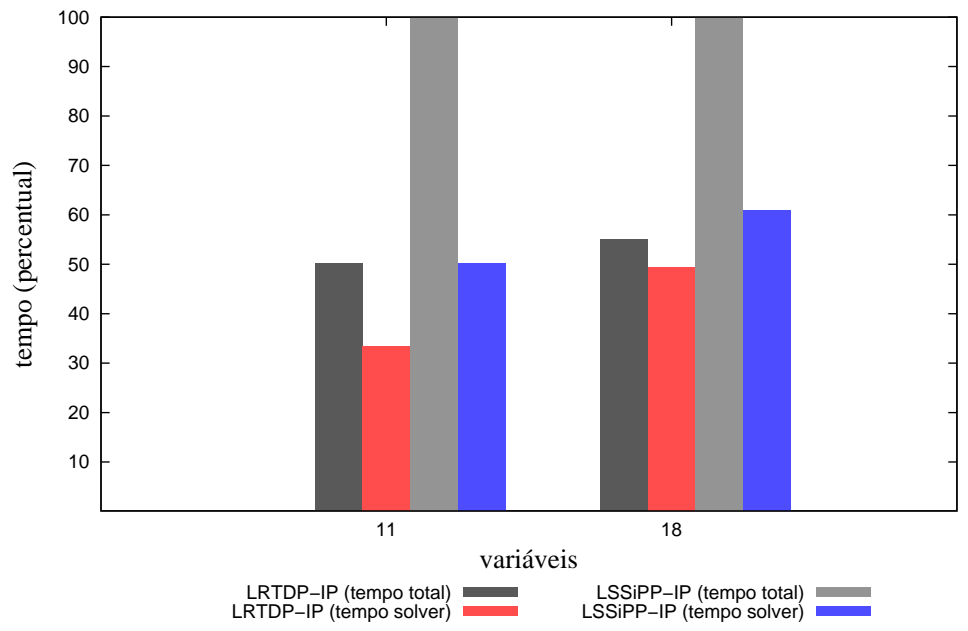

Figura 7.7: Tempo percentual de chamadas ao solver multilinear em relação ao tempo total para os algoritmos LSSiPP-IP $(t=5)$ e LRTDP-IP para o dominio NoRelaxed Triangle Tireword. 


\section{Capítulo 8}

\section{Conclusão e Trabalhos Futuros}

Neste trabalho foi proposto um algoritmo de programação dinâmica em tempo real que explora o conhecimento do estado inicial de um SSP MDP-IP especificado em termos de restrições gerais que podem também ser aplicados em SSP MDP-IPs fatorados. Observando a literatura atual, até então nenhum algoritmo assíncrono para os MDP-IPs havia sido proposto. Inicialmente, foi proposto um algoritmo enumerativo chamado RTDP-IP, e foi provado que o mesmo converge para valores ótimos, se o valor inicial de todos os estados é admissível e o método de amostragem não evita nenhum estado.

Também foi definido o algoritmo LRTDP-IP e motivados pela eficiência do SPUDD-IP, também foram propostas duas versões fatoradas do algoritmo RTDP-IP, chamadas de factRTDP-IP e factLRTDP-IP. Estes algoritmos assíncronos fatorados combinam o melhor do SPUDD-IP e do $R T D P-I P$ enumerativo: eles representam a função valor de forma eficiente e visitam somente os estados alcançáveis a partir do estado $s_{0}$. Porém nos experimentos o LRTDP-IP mostrou ser duas vezes mais rápido que o factLRTDP-IP.

Para domínios esparsos como o Navigation e Triangle Tireworld, o LRTDP-IP e o factLRTDP$I P$ são mais rápidos em até três ordens em relação ao SPUDD-IP. Adicionalmente, enquanto o SPUDD-IP não é capaz de devolver uma política para instâncias com mais de 20 variáveis, $o$ LRTDP-IP e o factLRTDP-IP devolvem políticas ótimas para instâncias com até 121 variáveis no dominio Navigation e com até 80 variáveis no dominio Triangle Tireworld.

Outro resultado importante para o factLRTDP-IP é que se o número máximo de pais nas redes credais dinâmicas é pequeno e o número de vértices de cada conjunto credal é pequeno, não existe nenhum custo significativo extra para calcular os vértices dos conjuntos credais e gerar os pontos aleatórios para os métodos predefined_parameter_choice e rand_parameter_choice, o que resulta em nenhuma diferença no tempo de execução em relação a amostragem utilizando os métodos minimax_parameter_choice, predefined_parameter_choice ou rand_parameter_choice.

Como esperado, em domínios densos o SPUDD-IP tem uma taxa de convergência melhor comparada aos algoritmos assíncronos propostos. Isto se deve ao fato de, em domínios densos, um número grande de estados são alcançados a partir do estado inicial, fazendo com que não haja vantagens em explorar o espaço de estados de forma assíncrona.

No caso dos algoritmo LSSiPP-IP, o mesmo apresenta pior desempenho que o LRTDP-IP, não replicando o comportamento do LSSiPP em relação ao LRTDP para SSP MDPs. Uma possível razão é que esta versão realiza mais chamadas ao solver que o LRTDP-IP, consumindo mais tempo. Outra possível razão pode ser o fato de ter sido utilizada uma função valor inicial muito simples. 


\subsection{Resumo das Contribuições}

Dada a motivação inicial de resolver problemas reais modelando-os como SSP MDP-IPs, este trabalho resultou em uma série de contribuições para a área de Inteligência Artificial, em especial na subárea de Planejamento Probabilístico:

- Na Seção 6.1, foi proposta a primeira solução assíncrona para SSP MDP-IPs, chamado RTDPIP. É mostrado, teoricamente (Seção 6.1.2) e empiricamente (Seção 7.3), que apesar de todas as imprecisões sobre as probabilidades de transição, o algoritmo RTDP-IP também converge para uma solução robusta;

- Foi proposta uma extensão do RTDP-IP, chamada LRTDP-IP (Seção 6.2) também baseado em uma solução existente para SSP MDP que melhora o tempo de convergência em relação ao RTDP-IP;

- Foram propostas duas versões simbólicas dos algoritmos: o factRTDP-IP e o factLRTDP-IP (Seções 6.3 e 6.4), que usam diagramas de decisão parametrizados (PADDs) para representar e atualizar eficientemente a função valor.

- Foi proposto um algoritmo assíncrono para Short-Sighted SSP MDP-IPs, chamado SSiPPIP e uma extensão deste algoritmo, chamado LSSiPP-IP (Seção 6.5), ambos baseados em versões para Short Sighted SSP MDPs. Neles, um SSP MDP-IP é particionado em diversos subproblemas e resolvido em partes, um subproblema de cada vez;

- No Capítulo 7, é mostrado empiricamente que os algoritmos LRTDP-IP e factLRTDP-IP tem uma velocidade de convergência em três ordens de magnitude maior que as soluções exatas estado-da-arte para domínios com matrizes de transição que não são densas e que permitem que instâncias grandes de problemas sejam resolvidas, algo que os algoritmos síncronos não eram capazes de fazer. Também é mostrado empiricamente que o LSSiPP-IP não consegue superar o LRTDP-IP.

\subsection{Trabalhos Futuros}

Entre os possíveis trabalhos futuros estão:

- Adaptação dos algoritmos para considerar dead-ends genéricos: um conceito muito comum em problemas de planejamento é o conceito de deadends (beco sem saída), um conjunto de estados em que, caso o agente os alcance, não poderá alcançar mais os estados meta. Se o problema tem becos sem saída, algoritmos como o SPUDD-IP não convergem para o valor ótimo e algoritmos como o RTDP-IP e factLRTDP-IP correm o risco de ficar infinitamente em um trial e não terminar (Kolobov et al., 2010). Note que, dois dos domínios usados nos experimentos neste trabalho tem becos sem saída, porém eles podem ser identificados facilmente verificando se ao executar uma ação no estado, é possível ou não ir para um outro estado. Para domínios com becos sem saída mais complexos, esta verificação é insuficiente. Portanto há necessidade de estudar outros métodos de deteç̧ão de dead-ends como os propostos por (Kolobov et al., 2010); 
- Propor novas funções valor admissíveis para Short-Sighted SSP MDP-IPs: com essas novas funções valor podem ser realizados novos experimentos com o LSSiPP-IP em diferentes domínios e com diferentes parâmetros $t$, para determinar se é possível encontrar um ganho na resolução de SSP MDP-IPs através do particionamento em Short-Sighted SSP MDP-IPs.

- Adaptar outros algoritmos assíncronos de SSP MDPs para os SSP MDP-IPs: assim como neste trabalho foi proposto uma extensão do (L)RTDP para SSP MDP-IPs chamada (L)RTDP-IP, posteriormente outras extensões podem ser propostas, baseadas por exemplo, no BRTDP e o no LAO*.

- Investigar abordagens Bayesianas para SSP MDP-IPs: recentemente apareceram alguns trabalhos que usam o conceito de robustez "soft" (Chen e Bowling, 2012, Delage e Mannor, 2010) e que adotam algum conhecimento sobre a distribuição dos conjuntos de probabilidades (abordagem Bayesiana). É interessante analisar algoritmos propostos nesta área para identificar pontos que possam ser usados nos algoritmos propostos neste trabalho de forma a melhorá-los. 


\section{Referências Bibliográficas}

Avis(2000) D. Avis. LRS: A revised implementation of the Reverse Search Vertex Enumeration algorithm, páginas 177-198. Birkhauser-Verlag.

Bahar et al.(1993) R. Iris Bahar, Erica A. Frohm, Charles M. Gaona, Gary D. Hachtel, Enrico Macii, Abelardo Pardo e Fabio Somenzi. Algebraic decision diagrams and their applications. Em Proceedings of 1993 IEEE/ACM International Conference on Computer-Aided Design, páginas 188-191. ISBN 0-8186-4490-7.

Barto et al.(1995) Andrew G. Barto, Steven J. Bradtke e Satinder P. Singh. Learning to act using real-time dynamic programming. Artificial Intelligence, 72:81 - 138. ISSN 0004-3702.

Bertsekas(1982) Dimitri P. Bertsekas. Distributed dynamic programming. IEEE Transactions on Automatic Control, 27:610-617.

Bertsekas e Tsitsiklis(1991) Dimitri P. Bertsekas e John N. Tsitsiklis. An analysis of stochastic shortest path problems. Math. Oper. Res., 16(3):580-595. ISSN 0364-765X.

Bertsekas e Tsitsiklis(1996) Dimitri P. Bertsekas e John N. Tsitsiklis. Neuro-Dynamic Programming. Athena Scientific Belmont, MA.

Bonet e Geffner(2003) B. Bonet e H. Geffner. Labeled RTDP: Improving the convergence of real-time dynamic programming. Proceedings of 2003 International Conference on Automated Planning and Scheduling, páginas 12-21.

Boutilier et al.(1996) Craig Boutilier, Nir Friedman, Moises Goldszmidt e Daphne Koller. Context-specific independence in Bayesian networks. Em Proceedings of the Twelfth International Conference on Uncertainty in artificial intelligence, páginas 115-123.

Boutilier et al.(1999) Craig Boutilier, Steve Hanks e Thomas Dean. Decision-theoretic planning: Structural assumptions and computational leverage. Journal of Artificial Intelligence Research, 11:1-94. ISSN 1076-9757.

Bryant(1992) Randal E. Bryant. Symbolic Boolean manipulation with ordered binary-decision diagrams. ACM Computing Surveys, 24:293-318.

Bryce et al.(2010) Daniel Bryce, Michael Verdicchio e Seungchan Kim. Planning interventions in biological networks. ACM Transactions on Intelligent Systems and Technology, 1(2):1-11.

Buffet e Aberdeen(2006) O. Buffet e D. Aberdeen. Policy-gradient for robust planning. Em Proceedings of the ECAI'06 Workshop on Planning, Learning and Monitoring with Uncertainty and Dynamic Worlds (PLMUDW'06).

Buffet e Aberdeen(2005) Olivier Buffet e Douglas Aberdeen. Robust planning with LRTDP. Em Proceedings of 2005 International Joint Conference on Artificial Intelligence, páginas 1214-1219.

Chen e Bowling(2012) Katherine Chen e Michael Bowling. Tractable objectives for robust policy optimization. Em Advances in Neural Information Processing Systems, páginas 2069-2077. 
Cozman(2000) F. G. Cozman. Credal networks. Artificial Intelligence, 120:199-233.

Cozman(2005) F. G. Cozman. Graphical models for imprecise probabilities. International Journal of Approximate Reasoning, 39(2-3):167-184.

Cui et al.(2006) Shulin Cui, Jigui Sun, Minghao Yin e Shuai Lu. Solving Uncertain Markov Decision Problems: An Interval-Based Method. Em Proceedings ICNC (2), páginas 948-957.

Cunha(2000) M. Cristina C. Cunha. Métodos Numéricos. Editora Unicamp.

Datta et al.(2003) Aniruddha Datta, Ashish Choudhary, Michael L. Bittner e Edward R. Dougherty. External control in Markovian genetic regulatory networks. Machine Learning, 52 (1-2):169-191.

Dean e Kanazawa(1990) Thomas Dean e Keiji Kanazawa. A model for reasoning about persistence and causation. Computational Intelligence, 5:142-150. ISSN 0824-7935.

Delage e Mannor(2010) Erick Delage e Shie Mannor. Percentile optimization for Markov decision processes with parameter uncertainty. Operations research, 58(1):203-213.

Delgado et al.(2010) Karina Valdivia Delgado, Cheng Fang, Scott Sanner e Leliane Nunes de Barros. Symbolic bounded real-time dynamic programming. Em SBIA, páginas 193-202.

Delgado et al.(2011) Karina Valdivia Delgado, Scott Sanner e Leliane Nunes de Barros. Efficient solutions to factored MDPs with imprecise transition probabilities. Artificial Intelligence, 175: 1498 - 1527. ISSN 0004-3702.

Devroye(1986) Luc Devroye. Non-Uniform Random Variate Generation. Springer-Verlag.

Drougard et al.(2013) Nicolas Drougard, Florent Teichteil-Konigsbuch, Jean-Loup Farges e Didier Dubois. Qualitative possibilistic mixed-observable MDPs. Em Proceedings of the Conference on Uncertainty in Artificial Intelligence (UAI), páginas 192-201. Association for Uncertainty in Artificial Intelligence.

Feng e et al.(2002) Zhengzhu Feng e et al. Symbolic LAO* search for factored Markov decision processes. Em Proceedings of the AIPS-02 Workshop on Planning via Model Checking, páginas $49-53$.

Feng et al.(2003) Zhengzhu Feng, Eric A. Hansen e Shlomo Zilberstein. Symbolic generalization for on-line planning. Em Proceedings of the 19th International Conference on Uncertainty in Artificial Intelligence, páginas 209-216. URL http://rbr.cs.umass.edu/shlomo/papers/FHZuai03.html.

Givan et al.(2000) R. Givan, S. Leach e T. Dean. Bounded-parameter Markov decision processes. Artificial Intelligence, 122:71-109(39).

Guestrin et al.(2003) Carlos Guestrin, Daphne Koller, Ronald Parr e Shobha Venkataraman. Efficient solution algorithms for factored MDPs. Journal of Artificial Intelligence Research, 19: 399-468.

Hoey et al.(1999) Jesse Hoey, Robert St-Aubin, Alan Hu e Craig Boutilier. SPUDD: Stochastic Planning using Decision Diagrams. Em Proceedings of the Fifteenth Conference on Uncertainty in Artificial Intelligence, páginas 279-288.

Hoffmann e Brafman(2005) Jörg Hoffmann e Ronen I. Brafman. Contingent planning via heuristic forward search witn implicit belief states. Em Proceedings of the Fifteenth International Conference on Automated Planning and Scheduling (ICAPS), páginas 71-80.

Holguin(2013) Mijail Gamarra Holguin. Planejamento probabilístico usando programação dinâmica assíncrona e fatorada. Dissertação de Mestrado, IME-USP. 
Howard(1960) Ronald A. Howard. Dynamic Programming and Markov Process. The MIT Press. ISBN 0262080095 .

Iyengar(2004) G. Iyengar. Robust dynamic programming. Mathematics of Operations Research, $30: 257-280$.

Kolobov et al.(2010) Andrey Kolobov, Mausam e Daniel Weld. Sixthsense: Fast and reliable recognition of dead ends in MDPs. Em Twenty-Fourth AAAI Conference on Artificial Intelligence.

Kolobov et al.(2012) Andrey Kolobov, Peng Dai, Mausam e Daniel Weld. Reverse iterative deepening for finite-horizon MDPs with large branching factors. Proceedings of the 2012 International Conference on Automated Planning and Scheduling.

Little e Thiébaux(2007) Iain Little e Sylvie Thiébaux. Probabilistic planning vs. replanning. Em ICAPS Workshop on IPC: Past, Present and Future.

McMahan et al.(2005) H. Brendan McMahan, Maxim Likhachev e Geoffrey J. Gordon. Bounded Real-Time Dynamic Programming: RTDP with monotone upper bounds and performance guarantees. Em Proceedings of the 22nd International Conference on Machine Learning, páginas 569-576. ISBN 1-59593-180-5.

Murtagh e Saunders(1978) Bruce A Murtagh e Michael A Saunders. Large-scale linearly constrained optimization. Mathematical programming, 14(1):41-72.

Nilim e El Ghaoui(2005) Arnab Nilim e Laurent El Ghaoui. Robust control of Markov decision processes with uncertain transition matrices. Operations Research, 53(5):780-798.

Pal et al.(2008) R. Pal, A Datta e E.R. Dougherty. Robust intervention in probabilistic Boolean networks. IEEE Transactions on Signal Processing, 56(3):1280-1294.

Patek e Bertsekas(1999) Stephen D Patek e Dimitri P Bertsekas. Stochastic shortest path games. SIAM Journal on Control and Optimization, 37(3):804-824.

Puterman(1994) Martin L. Puterman. Markov Decision Processes: Discrete Stochastic Dynamic Programming. John Wiley \& Sons.

Roy(2004) Benjamin Van Roy. Approximate Dynamic Programming. Class lecture from University of Washington, 2004.

Sabbadin(1999) Régis Sabbadin. A possibilistic model for qualitative sequential decision problems under uncertainty in partially observable environments. Em Proceedings of the Conference on Uncertainty in Artificial Intelligence (UAI), páginas 567-574.

Sanner et al.(2009) Scott Sanner, Robby Goetschalckx, Kurt Driessens e Guy Shani. Bayesian real-time dynamic programming. Em Proceedings of 2009 International Joint Conference in Artificial Intelligence, páginas 1784-1789.

Satia e Lave Jr.(1970) Jay K. Satia e Roy E. Lave Jr. Markovian decision processes with uncertain transition probabilities. Operations Research, 21:728-740.

Smith e Simmons(2006) Trey Smith e Reid G. Simmons. Focused real-time dynamic programming for MDPs: Squeezing more out of a heuristic. Em Proceedings of the National Conference on Artificial Intelligence (AAAI).

St-Aubin et al.(2000) Robert St-Aubin, Jesse Hoey e Craig Boutilier. APRICODD: Approximate policy construction using decision diagrams. Em Proceedings of Advances in Neural Information Processing Systems, páginas 1089-1095. 
Sutton e Barto(1998) Richard S. Sutton e Andrew G. Barto. Introduction to Reinforcement Learning. MIT Press, Cambridge, MA, USA, 1st edição.

Trevizan(2013) Felipe W Trevizan. Short-sighted Probabilistic Planning. Tese de Doutorado, Carnegie Melon.

Trevizan et al.(2007) Felipe W. Trevizan, Fabio G. Cozman e Leliane N. de Barros. Planning under risk and Knightian uncertainty. Em Proceedings of International Joint Conferences on Artificial Intelligence, páginas 2023-2028, Hyderabad, India.

White III e El-Deib(1994) Chelsea C. White III e Hank K. El-Deib. Markov decision processes with imprecise transition probabilities. Operations Research, 42:739-749.

Wiesemann et al.(2013) Wolfram Wiesemann, Daniel Kuhn e Berç Rustem. Robust Markov decision processes. Mathematics of Operations Research, 38(1):153-183.

Witwicki et al.(2013) Stefan J. Witwicki, Francisco S. Melo, Jesus Capitan e Matthijs T. J. Spaan. A flexible approach to modeling unpredictable events in MDPs. Em Proceedings of the Twenty-Third International Conference on Automated Planning and Scheduling, ICAPS, páginas 260-268.

Yin et al.(2007) Minghao Yin, Jianan Wang e Wenxiang Gu. Solving Planning Under Uncertainty: Quantitative and Qualitative Approach. Em Proceedings IFSA (2), páginas 612-620.

Zhang e Poole(1994) N. L. Zhang e D. Poole. A simple approach to Bayesian network computations. Em Proceedings of the Tenth Canadian Conference on Artificial Intelligence, páginas 171-178. 


\section{Apêndice I - Convergência do RTDP-IP para MDP-IPs de Horizonte Infinito}

Neste apêndice será mostrada a prova de convergência do algoritmo RTDP-IP para MDP-IPs de horizonte infinito. Esta prova também é baseada na prova de Barto et al. (1995) (Seção 3.2.1) e nas notas de aula de Roy (2004).

Neste caso, as funções $Q(s, a)$ e $V(s)$ são representadas como:

$$
V^{t+1}(s)=\max _{a \in A}\left\{Q^{t+1}(s, a)\right\}
$$

em que:

$$
Q^{t+1}(s, a)=R(s, a)+\gamma \min _{P \in K} \sum_{s^{\prime} \in S} P\left(s^{\prime} \mid s, a\right) V^{t}\left(s^{\prime}\right) .
$$

Para provar que o RTDP-IP converge, primeiro é necessário mostrar que a programação dinâmica assíncrona (Definição 3.2.3) converge para MDP-IPs.

Teorema 1. A programação dinâmica assincrona para MDP-IPs com horizonte infinito e fator de desconto converge para $V^{*}$ se os valores de cada estado forem atualizados infinitas vezes.

Prova: Como as funções de atualização para MDP-IPs com horizonte infinito e fator de desconto $Q(s, a)$ e $V(s)$ (Equações 1 e 2) são contrações com respeito a norma do supremo (sup-norm) (Iyengar, 2004, Nilim e El Ghaoui, 2005), esta condição é o suficiente para a convergência da programação dinâmica assíncrona para MDP-IPs (Bertsekas, 1982).

Como dito na Seção 3.2.1, o RTDP-IP pode ser visto como um caso especial da programação dinâmica assíncrona onde um único estado é atualizado de cada vez. Porém é necessário assumir uma profundidade de término de cada trial, visto que um MDP-IP de horizonte infinito e fator de desconto não tem estados meta. Fazendo a suposição que não há laços e deadends, pode-se assumir que essa profundidade máxima é ao menos igual $|S|$. Considerando um conjunto de estados iniciais $I=S$, é possível garantir que cada estado $s \in S$ seja atualizado infinitas vezes. Porém no caso $I=\left\{s_{0}\right\}$ ou qualquer $I \subset S$, não é possível garantir que todos os estados alcançáveis sob uma política ótima sejam atualizados, assim o Teorema 1 não pode ser usado diretamente para provar a convergência do RTDP-IP.

Neste caso, assim como na prova de convergência do RTDP para MDPs, é necessário mostrar que o RTDP-IP realiza operações de programação dinâmica assíncrona sobre o conjunto de estados relevantes. A idéia chave desta prova é mostrar que a admissibilidade da função valor de um MDP-IP com fator de desconto é preservada durante os trials do RTDP-IP.

Proposição 1. Se o valor inicial $V^{0}$ é admissivel (i.e., for um limite superior), a admissibilidade da função valor é preservada durante a execução do RTDP-IP, i.e., se $V^{0}(s) \geq V^{*}(s), \forall s \in S$ então $V^{t}(s) \geq V^{*}(s), \forall s \in S$ e $t \in \mathbb{N}^{*}$.

Prova: Seja $s_{t}$ o estado atual, $a_{t}$ a ação gulosa e $V^{t}$ a função valor no instante de tempo $t$, gerada pela execução do RTDP-IP começando de um estado inicial arbitrário. É verdade que 
$V^{t}(s) \geq V^{*}(s), \forall s \in S$ pois $V^{t}(s)=V^{t-1}(s), \forall s \neq s_{t}$ e por indução se $V^{t-1}\left(s^{\prime}\right) \geq V^{*}\left(s^{\prime}\right), \forall s^{\prime} \in S$, então para todo $t$ :

$$
\begin{aligned}
V^{t}\left(s_{t}\right) & =\max _{a \in A}\left\{R\left(s_{t}, a\right)+\gamma \min _{P \in K}\left\{\sum_{s^{\prime} \in S} P\left(s^{\prime} \mid s_{t}, a\right) V^{t-1}\left(s^{\prime}\right)\right\}\right\} \\
& \geq \max _{a \in A}\left\{R\left(s_{t}, a\right)+\gamma \min _{P \in K}\left\{\sum_{s^{\prime} \in S} P\left(s^{\prime} \mid s_{t}, a\right) V^{*}\left(s^{\prime}\right)\right\}\right\}=V^{*}\left(s_{t}\right)
\end{aligned}
$$

Teorema 2. (Convergência do RTDP-IP) Em um MDP-IP com fator de desconto, repetidos trials do RTDP-IP irão eventualmente gerar valores ótimos sobre todos os estados relevantes se: (i) a função valor inicial é admissivel, i.e., $V^{0}(s) \geq V^{*}(s)$ e (ii) o método para escolha de $P \in K$ para amostrar o próximo estado garanta a visita de todos os próximos estados eventualmente.

Prova: Suponha que o conjunto de estados $S$ seja dividido em duas partições. Seja $L$ o conjunto de todos os estados visitados frequentemente e $\bar{L}=S \backslash L$ o conjunto de estados visitados um número finito de vezes. Sabendo que existem um número finito de estados e um número finito de ações, durante os trials do RTDP-IP deve existir um tempo $\hat{t}$ em que somente são escolhidas ações que tem probabilidade zero de ir para um estado que está em $\bar{L}$ (considere $B$ o conjunto destas ações), assim o RTDP-IP permanecerá visitando os estados em $L$. Visto que após $\hat{t}$ cada estado em $L$ é atualizado infinitas vezes, sabe-se que o algoritmo irá convergir sobre este conjunto (i.e. para $s \in L$, $V^{t}(s)$ converge para $V_{L}^{*}(s)$, onde $V_{L}^{*}(s)$ é relacionado somente com ações pertencentes a $\left.B\right)$.

Sejam $V_{L}^{*}(s)=V_{\pi}(s)$, onde $V_{\pi}(s)$ é a função valor para cada $s \in S$ associados com alguma política $\pi$. Como $V^{*}(s)$ é o valor máximo para todas as políticas, $V_{\pi}(s) \leq V^{*}(s), \forall s \in L$. Adicionalmente, pela Proposição 1 é conhecido que $V_{\pi}(s) \geq V^{*}(s), \forall s \in L$, o que implica em:

$$
V_{L}^{*}(s)=V_{\pi}(s)=V^{*}(s), \forall s \in L
$$

Assim, é possível concluir que $L$ é o conjunto de estados relevantes, $\pi=\pi^{*}$ e $B$ são as ações ótimas sobre $L$.

Note que, durante os trials do RTDP-IP deve existir um tempo $t_{1} \geq \hat{t}$ tal que, $\forall t \geq t_{1}$ temos $s_{t} \in L$ e $a_{t} \in B$. Assim, neste instante temos $V_{L}^{*}(s)=V^{*}(s), \forall s \in L$ e $V_{\bar{L}}(s) \geq V^{*}(s), \forall s \in \bar{L}$. Entretanto é preciso garantir que $V_{L}^{*}(s)$ não irá mudar visto que, por definição, as ações em $B$, quando aplicadas nos estados em $L$, não levam a estados em $\bar{L}$.

Também, para garantir que os estados relevantes são atualizados infinitas vezes, é necessário garantir que o método de escolha de $P \in K$ para amostrar o próximo estado nunca eliminará um estado que precisará ser visitado.

Para completar a prova de convergência, é necessário garantir que todos os estados relevantes serão visitados infinitas vezes. Para isto, o método que escolhe $P \in K$ para amostrar o próximo estado nunca deverá eliminar nenhum estado que possa eventualmente ser visitado. Nesta proposta, visto que rand_parameter_choice escolhe um $P \in K$ diferente para cada amostragem do próximo estado, ele eventualmente irá visitar todos os estados alcançáveis. Semelhante a prova de convergência do RTDP-IP para SSP MDP-IPs, para os outros métodos (minimax_parameter_choice e predefined_parameter_choice), caso eles escolham $P\left(s^{\prime} \mid s, a\right) \in K(\cdot \mid s, a)$ igual a 0 , é necessário escolher outro $P\left(s^{\prime} \mid s, a\right) \in K(\cdot \mid s, a)$ diferente de 0 para algum $s^{\prime} \in S$ a cada vez que se visita o estado $s$ com a ação $a$ utilizando a Equação 6.1. Assim, em geral o Teorema 2 deve ser reescrito como:

Teorema 3. (Convergência do RTDP-IP para diferentes métodos de amostragem para o próximo estado) Em um MDP-IP com fator de desconto, repetidos trials do RTDP-IP irão eventualmente gerar valores ótimos sobre todos os estados relevantes se: (i) a função valor inicial é admissivel, i.e., $V^{0}(s) \geq V^{*}(s)$ e (ii) o método para escolha de $P\left(s^{\prime} \mid s, a\right) \in K(\cdot \mid s, a)$ para amostrar o próximo estado garanta nunca escolher $P\left(s^{\prime} \mid s, a\right) \in K(\cdot \mid s, a)$ igual a 0 , para algum $s^{\prime} \in S$ dado $(s, a)$. 


\section{Apêndice II - Descrição das ferramentas utilizadas nos experimentos}

Os programas implementados para os experimentos deste trabalho foram baseados nas implementações iniciais da biblioteca RDDLSIM ${ }^{1}$, utilizada no IPPC-2011, e no trabalho de Delgado et al. (2011). O código fonte destes programas e a Wiki com documentações atualizadas para utilização deles estão disponíveis na plataforma Google Code sobre o projeto MDPIP, através dos seguintes links:

- Projeto: https://code.google.com/p/mdp-ip

- Wiki: https://code.google.com/p/mdp-ip/wiki/Index

O projeto está escrito na linguagem Java 6 e utiliza as bibliotecas externas: (i) LRS (Avis, 2000) para enumeração dos vértices dos politopos; (ii) MINOS (Murtagh e Saunders, 1978), solver não-linear, chamado através do AMPL; e (iii) grappa para a geração interna de grafos.

No código fonte, os seguintes pacotes são importantes para realizar experimentos:

- add: pacote com classes para manipular os ADDs e PADDs.

- generator: pacote com classes para geração de instâncias de problemas utilizados nos experimentos, como o Navigation (classe NavigationGen), Triangle Tireworld (classes TriangleTireWorldGen e RelaxedTriangleTireWorldGen) e SysAdmin (classe SSPSysAdminUniRingGen).

- $m d p$ : pacote com as implementações internas dos algoritmos utilizados neste trabalho.

- mdp.algorithms: pacote com as classes de ponto de entrada (i.e., classes que serão chamadas diretamente pelo executável do java) com as devidas parametrizações.

Para executar um experimento, é necessário chamar uma classe que representa um algoritmo do pacote $m d p$.algorithms, que implementa a interface de entrada, com as devidas parametrizações do algoritmo. Neste pacote, os seguintes algoritmos estão disponíveis, com suas respectivas classes:

- SSiPP-IP (classe SSiPP)

- LSSiPP-IP (classe LabeledSSiPP)

- RTDP-IP (classe RTDPIPEnum)

- factRTDP-IP (classe RTDPIP)

- LRTDP-IP (classe LRTDPIPEnum)

- factLRTDP-IP (classe LRTDPIP)

- SPUDD-IP (classe SPUDDIP)

\footnotetext{
${ }^{1}$ Disponível em https://code.google.com/p/rddlsim/
} 
- Objective-IP (classe ObjectiveIP)

- Iteração de Valor (classe IV)

Para executar os experimentos em Windows ou Linux, é necessário modificar o arquivo config.properties do projeto, indicando na configuração user.os se o sistema operacional é Windows $($ user.os $=$ windows $)$ ou Linux $($ user.os $=$ linux $)$, informar qual é o diretório raiz onde se encontra o projeto através da configuração root.dir e atualizar a variável PATH do sistema operacional para encontrar as dependências externas para o LRS e AMPL através dos seguintes comandos:

- Windows: \%PATH\% : ROOTDIR $\backslash$ libs $\backslash$ windows

- Linux: \$PATH : ROOTDIR/libs/linux

Onde ROOTDIR deve ser substituído pelo diretório raiz onde se encontra o projeto. 